UNIVERSIDADE DE SÄO PAULO

INSTITUTO DE FÍSICA

\title{
PARTÍCULAS E CORDAS EM ESPACOS COM MÉTRICAS DEGENERADAS
}

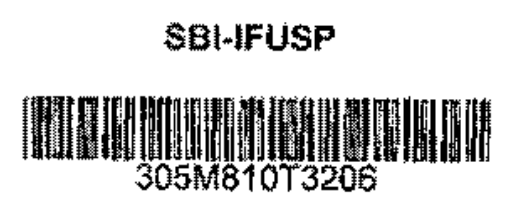

LUÍS ANTONIO CABRAL

Banca Examinadora;

Prof Dr. Marcelo Otảyio Caminha Comes (IFUSP)

Prof. Dr. Adilson José da Silva (IFUSP)

Prof. Dr. Nathan Jacob Berkovits (IFT/UNESP)

Prof. Dr. João Barcelos Neto (UFRJ)
Tese de Doutorado submetida ao Institu to de Fisica da Universidade de Säo Paulo

OrIextrado: Prof, DR, Victor de ÓlivelRA Rivelles
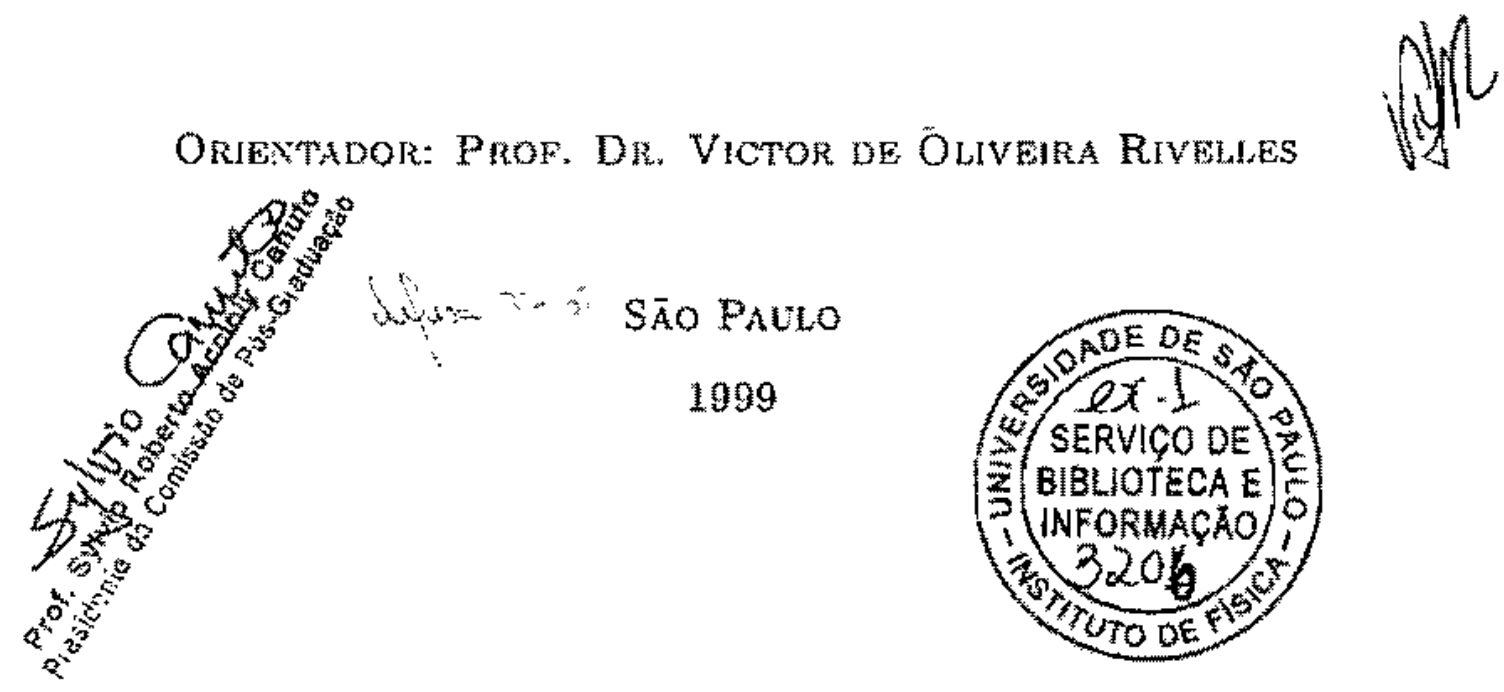


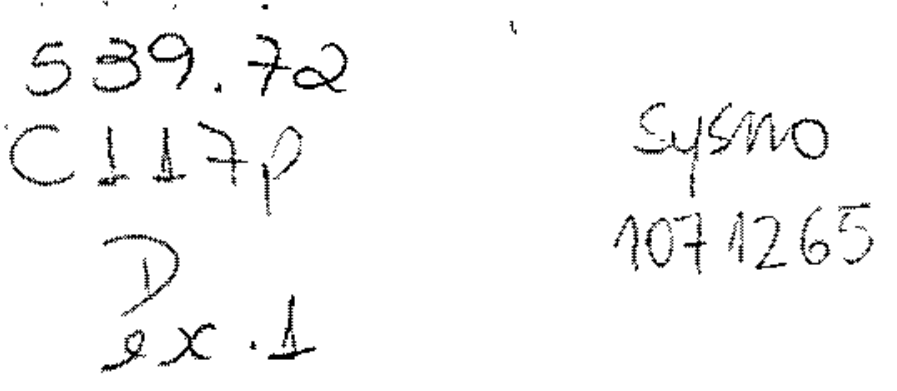

FICHA CATALOGRÁFICA

Preparada pelo Serviço de Biblioteca e Informação do Instituto de Física da Universidade de São Paulo

Cabral, Luis Antonio

Particulas e Cordas em Espaços com Métricas

Degeneradas. São Paulo, 1999.

Tese (Doutoramento) - Universidade de Săo Paulo. Instituto de Física - Departamento de Fisica Matemática

Orientador: Prof. Dr. Victor de Oliveira Rivelles

Área de Concentraçăo: Física das Particulas Elementares e Campos

Unitermos: 1. Sistemas Vinculados; 2. Particulas;

3. Cordas.

USP/IF/SBi-052/99 


\section{Resumo}

Neste trabalho estudamos a dinâmica de partículas e cordas num campo gravitacio. nal com métrica degenerada. Utilizamos o formalismo Hamiltoniano para determinar os vínculos da teoria e a dinämica efetiva do sistema. Classificamos a estrutura dos vínculos de acordo com a forma da métrica e dimensão do espaço(-tempo). Obtemos também a representação das simetrias em termos de isometrias geradas por auto-vetores nulos da métrica. 


\begin{abstract}
In this work we study the dynamics of particles and strings in a gavitational field with degenerate metric. We use the Hamilonian formalism to find out the constraints and the effective dynamics of the system. We classify the constraint structure according to the form of the metric and the space(time) dimension. We also obtain the representation of the symmetres in terms of the isometries generated by the null eigenvectors of the metric.
\end{abstract}




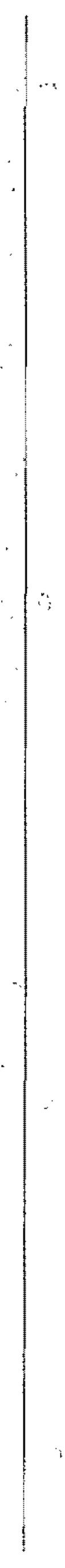




\section{Agradecimentos}

É com grande satisfaçào que chego a esta etapa da minha formaçâ para prestar a muitas pessoas os meus sinceros agradecimentos.

Agradeço com muita satisfaçäo:

Ao men orientador, o Prof. Rivelles, que me acompanhou en todas as fases deste trabalho, prestando toda ajucla necessária nos momentos mais importantes do projeto;

Aos funcionatrios do departamento pelo auxilio durante a minha permanência no IFUSP:

Aos colegas de vários projetos - Leênidas, Mônica, Otávio, Marcelo, Luiz, Cândido e outros;

As amigas que to deram incentivo constante - Sandra, Joana, Lilian, Silvia, Andréia, Dolores e Stela;

A todos os meus familiares que me transmitiram alegria e esperança;

A Camila e à Clara pela dedicaçäo e paciência em toda a nossa existência;

A FAPESP pelo auxílio financeiro. 


\section{Conteúdo}

1 Introduçăo 3

2 A Particula Nâno Relativistica 8

2.1 A Partícula em Campo de Fundo Gravitacional Näo Degenerado . . . . . 9

2.2 A Partícula num Campo de Fundo Gravitacional Degenerado . . . . . . . 11

2.3 Vetores de Base Integrávois $\ldots \ldots \ldots \ldots \ldots$

2.4 Vetores de Base Năo Integráveis . . . . . . . . . . . . . 15

$2.4 \ldots \quad D \ldots r \mathrm{Par} \ldots \ldots \ldots \ldots \ldots$

$2.4 .2 \quad D-r \operatorname{lnpar} \ldots \ldots \ldots \ldots$

2.5 Caso $N_{p}=0 \ldots \ldots \ldots \ldots \ldots \ldots$

2.6 Isometrias e Vinculos de Primeira Clase com Métrica Degeneradas . . . 20

3 A Particula Relativistica

3.1 A Particula num Campo de Fundo Gravitacional näo Degenenado . . . . 24

3.2 A Partícula em Campo de Fundo Gravitacional Degenexado . . . . . . . 26

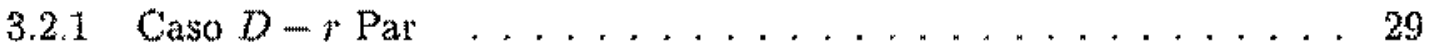

3.2 .2 Caso $D-r$ Ímpar $\ldots \ldots \ldots \ldots \ldots$

4 A Corda Bosónica

4.1 Algumas Solucoes Clássicas para a Corda em Campos de Fundo Gravitadionatis $\ldots \ldots \ldots \ldots \ldots$ 
4.2 Formalismo Hamiltoniano $\ldots \ldots \ldots \ldots \ldots \ldots \ldots \ldots$

4.2.1 Campo de Fundo Gravitacional näo Degenerado . . . . . . . 41

4.2 .2 Campo de Fundo Gravitacional Degenerado . . . . . . . . 43

4.2 .3 Caso $D-r$ Par . . . . . . . . . . . . . . 48

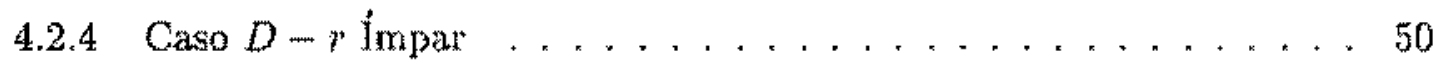

4.3 Isometrias na Corda com Métrica Degenerada e Vínculos de Primeira Classe 54

4.4 Invariăncia Conforme em Nível Quântico ............

5 Conclusăo 59

A Propriedades dos Auto-Vetores Nulos em $D-r$ Ímpar 61

B Deduçāo da Hamiltoniana Canônica da Corda com Métrica Degenerada e Vínculos Secundârios $\quad 65$

C Dedução da Álgebra dos Vínculos para a Corda 72 


\section{Capítulo 1}

\section{Introdução}

As singularidades têm um papel fundamental na relatividade geral [1]. Entretanto, näo estâ claro como incorporânlas em uma teoria quântica da gravitaçäo. As singularidades do espaço-tempo têm una característica importante na gravitaçào quântica como demonstrado en processos envolvendo a radiação térmica de Hawking. No entanto, o nosso conhecimento atual para descrever o processo da evaporação de buracos negros até a sua extinção ainda está incompleto. Porém, a situação é menos limitada no contexto da teoria de cordas. Neste caso, a entropia dos buracos negros extremos e quase-extremos, pode ser calculada considerando a presença de D-branas próximas ao horizonte de eventos [2]. No acoplamento fraco, estas D-branas säo descritas por uma teoria de gauge supersimétrica na qual é possivel contar os estados relevantes para entropia. Já que as D-branas estäo localizadas próximas ao horizonte, as singularidades no interior do buraco negro ainda näo foram tratadas completanente na teoria de cordas, porém, hấ algumas tentativas neste contexto [3].

As singularidades que dảo lugar aos buracos negros manifestam-se no tensor de curvatura. O efeito deste tipo de singularidade na evoluçāo quântica de partículas foi estudado recentemente em [4]. Neste caso, o argumento principal é que uma partícula movendo-se num espaço-tempo que é geodesicamente incompleto, possui uma evoluçăo quântica bem 
definida desde que o operador Hamiltoniano seja essencialmente auto-adjunto.

Hẫ, também, um outro tipo de singularidade em que o tensor de curvatura näo se torna singular. Isto acontece quando a métrica é degenerada, isto é, quando näo possui inversa. Neste caso, se a métrica é degenerada em um conjunto de medida zero, i.e., se ocorre em pontos isolados do espaço, então a curvatura nâo diverge e a topologia do espaço-tempo pode mudar [5]. Este tipo de singularidade é tambérn compatível com a estrutura causal do espaco-tempo [6].

As métricas degeneradas aparecem na relatividade geral na formulação de Palatíni, onde a açăo é definicla em temos das tetradas e da conexão de Lorentz. Nesta formulaçầ, a ação e as equaçós de movimento não dependem do inverso da tetrada e assim são bem definidas mesmo quando a tetrada é degenerada [5].

Ein uma outra situação, as métricas degeneradas também aparecem cotno métricas indusidas em hipersuperficies nulas [7], sendo a geometria da hipersuperfície considerada sob o ponto de vista do espaço alvo, ou seja, sob o ponto de vista extrínseco a hipersuperficle. Neste aspecto, a métrica do espaço alvo, que não é degenerada, se relaciona corn a mëtrica da hipersuperficie imersa neste espaço. Nesta condiçâo é possivel definir uma métrica inversa estendida para a hipersuperficie nula. Por outro lado, as mếtricas degeneradas podem ser consideradas sob o ponto de vista intrínseco, ou seja, consideradas exclusivamente sob a hipersuperficie em que estão definidas sem recorrer a imersōes em ešn paços com dimerıã̃o superior à hipersuperfície. Definidas desta forma, tornam-se métricas auxiliares para o estudo da radiaçäo gravitacional $[8]$, sendo que em quatro tlimensöes säo métricas de posto três.

Em outros contextos, ternos a presença de tetradas e as generalizaçoues destas para outras dimensöes, que denominam-se vielbeins, que também são degenerados. Neste aspecto, hat a formulacäo de Ashtekar da relatividade gerals que vtiliza um espaço de fase con variáveis complexas definidas por meio de uma teoria de gauge do tipo Yang-Mills 
com grupo $S O(3)$ [9]. O formalismo de Ashtekar permanece bem definido quando a métrica obtida na sua formulaçäo é degenerada. Devido a esta propriedade da métrica, muitas questöes relativas a esta formulação continuam sendo objeto de pesquisa recente [10]. Além da formulaçăo de Ashtekar, existem outras teorias da gravitaçăo em duas e três dimensöes que podem ser formuladas como teorias de gauge topológicas do tipo BF e de Chern-Simons [11]. Como um exemplo de uma teoria abeliana do tipo BF, devenos ter uma açäo em uma variedade $M$, com dimensâo arbitrária, independente do tensor mệtrico e escrita em ternos de campos na formulaçâo das formas diferencials. Esta açäo ira fornecer uma descriçäo da cohomologia de de Rham sobre M [12]. Estas teorias surgèn também como uma generalização da teoria de Chern-Simons para dimensöes majores que três, permitindo ohter generalizaçōes dos invariantes topológicos na teoria dos nós obtidos inicialmente ern [13]. As teorias topológicas estăo envolvidas numa descrição de uma fase topológica da gravitação quântica na qual a tetrada se anula e a invariância por difeomorfismo é quebrada [14]. Adicionalmente, as teorias topolögicas foram recentemente utilizadas com um meio auxiliar de se obter soluções degeneradas na relatividade geral [15].

No contexto da teoria de cordas foi demonstrado que cordas podem se propagar em campos de fundo gravitacionais degenerados. Quando as coordenadas da corda satisfazem deterninadas condiçoes a métrica é degenerada de posto um [16]. Sabemos, também, que p-branas portandone como instantons são descritas por uma métrica degenerada na hipersuperfície de evoluçāo [17]. Cordas com tensão nula [18] e D-branas corn tonsäo nula [19], sâo tambểm descritas por uma ação que envolve métricas degeneradas. Neste caso, a ação é construida na formulação de primeira ordem, ie, linear com respeito às derivadas das coordenadas do espaço alvo, sendo que a métrica auxiliar é degenerada e não o campo de fundo gravitacional. A forma da métrica degenerada é do tipo $g_{\mu}=E_{p}^{A} E_{p}^{B} \eta_{A B}$ com $E_{\mu}^{A}$ formando matrizes năo quadradas e retratando uma imersâa que implica num espaço 
tangente com métuca $n_{A}$ o dinansão menor que a da hipersuperficie de evolução.

Tudo o que consideramos até o momento indica que as métricas degeneradas podem ter um papel importante na gravitacâo quâtica. Num tratamento em nixel clássico a principal consequência das métricas degeneradas é permitir a mudança de topologia do

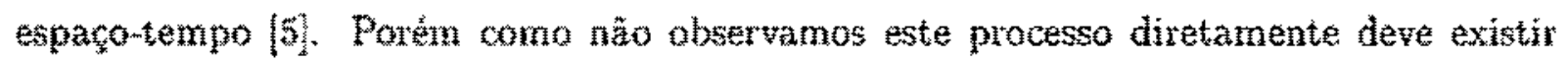
algum mecanismo que o suprime, provavelnante em nivel quantico. Por outro lado, o comportamento da mat́ria num espaco-tempo com nétrica degenerada näo tem sido propriamente explorado. Pasa o caso das teorias de campo topológicas não hả presença de métricas na açäo dos modelos, assim, espaço-tempos com métricas degeneradas, podem ser cousiderados nestas teorlass. No entanto, quando consideramos campos de matéria com texmos cinéticos, o acoplamento da gravitação requer a introduçāo de campos tensoriax́s contravariantes. O exemplo mais simples é a ação do campo escalar que depende do inverso da métrica. Para tratar estes casos foi proposto um procedimento [20] que não necessita de campos tensoriais contravariantes e assim săo obtidas açōes numa formulação esterdida que admite tetradas degeneradas, além de conter o limite original da teoria quando a tetrada 6 inversivel.

Una importante classe de matéria, que pode ser acoplada ao tensor métrico, é constituida por particulas e cordas [21]. A agäo as equaçoes de movimento das particulas e cordas năo dependem do inverso da métrica sầo bem definidas mesmo quando a métrica se torna dogenerada. Nesta tese, iremos considerar a dinâmica de partículas e cortas movendo-se em espacos ou espaco-tempos com ma métrica degenerada. Os espaços em estudo estüo associados a regioes com métrica degenerada em toda extensão. Analisaremos a estrutura los vinculos impostos pela métrica degenerada e encontraremos os graus de liberdade feftuts que deserevem o movimento de particulas e cordas. Desta forma cbteremos a dinamica efetiva para particulas e cordas em movimento num campo de furlo gravitacional degenerado. Iremos considerar inicialmente a particula näo relativística 
no Capítulo 2. Este capítulo será desenvolvido em detalhes, visto que a análise dos vínculos contida nos capítulos seguintes terāo alguns aspectos similares aos da partícula não relativística. Demonstramos, também, que as isometrias da métrica degenerada estāo associadas às simetrias locais geradas pelos vínculos de primeira classe. No Capítulo 3 estudaremos a partícula relativística e no Capítulo 4 a corda bosônica. Neste capítulo discutimos também alguns aspectos em aberto da quantização da corda em campos de fundo gravitacional degenerados. No Capítulo 5 apresentaremos algumas observaçōes finais e conclusōes, bem como algumas questões que poderão ser exploradas no futuro. 


\section{Capítulo 2}

\section{A Partícula Não Relativística}

\section{Introdução}

O modelo de uma partícula nazo relativística em campo de fundo gravitacional tern sido objeto de bastante pesquisa na literatura [22]. Quando a métrica clo espaço admite inversa, ou seja, não é degenerada, não há vínculos no sistema. Apesar disto, a açăo e as equaçōes de movimento não apresentan o inverso da métrica, de tal forma que possamos considerar nestas, o caso em que a métrica é degenerada. Porém, quando a métrica é degenerada, surgem vinculos já que neste caso năo podemos isolar todas as velocidades em função dos momentos canonicamente conjugados. Sendo assim, utilizamos o procedimento de Dirac [23] para o desenvolvímento do formalismo Hamiltoniano para sistemas vinculados e verificaremos que ainda surgern vínculos adicionais com una estrutura năo trivial.

O caso degenerado apresenta uma estrutura vica de vínculos. No formalismo Hamiltoniano, temos situações que dependem do posto da mëtrica e da dimensäo do espaço que influenciann na existência de uma determinada classe de vínculos. Esta estrutura de vínculos será bastante útil como modelo simplificado no tratamento dos vínculos do caso da corda em campo de fundo gravitacional.

Como discutimos na Introduçäo geral, o fato da métrica poder estar definida sem 
inversa na açăo e nas equaçōes de movimento de um modelo, abre uma linha de análise que terá resultados näo triviais, visto que para um espaço com métrica degenerada, o movimento de uma partícula neste espaço admite uma caracteristica notavelmente distinta do caso em que a métrica admite uma inversa. É o que veremos nas seções a seguir.

\subsection{A Partícula em Campo de Fundo Gravitacional Não Degenerado}

Uma partícula sujeita a um campo gravitacional é descrita pela açăo

$$
S=\frac{1}{2} m \int_{i_{1}}^{k_{2}} g_{i j} \dot{x}^{i} \dot{x}^{j} d t
$$

onde m é a massa da partícula. A partir desta ação as equaçoes de movimento obtidas รี้กั้

$$
g_{l j} \ddot{x}^{j}+\Gamma_{i j l} \dot{x}^{\dot{1}} \dot{x}^{j}=0
$$

onde

$$
\Gamma_{i j l}=\frac{1}{2}\left(\partial_{i} g_{j l}+\partial_{j} g_{i l}-\partial_{i} g_{i j}\right)
$$

Noternos que a açäo e as equaçôes de movimento estäo bern definidas mesmo que a métrica não posssua inversa. Ao considerarmos o momento canonicamente conjugado a $x^{i}$,

$$
P_{i}=\frac{\partial L}{\partial \dot{x}^{i}}=m \dot{x}^{j} g_{j}
$$

se $g_{i j}$ não for degenerada, temos que

$$
\dot{x}^{i}=\mathfrak{q}^{j i} \frac{P_{i}}{m}
$$


Com isto, podemos construir a Hamiltoniana canônica associada a esta ação a partir de $H_{c}=P_{i} \dot{x}^{i}-L$, obtendo

$$
H_{c}=\frac{1}{2 m} g^{i j} P_{i} P_{j} .
$$

Neste caso, o sistema não possui vínculos, o que possibilita estudar a evoluçāo da partícula utilizando $H_{c}$.

Adicionalmente, devemos notar que com o determinante de $g_{i j}$ sendo diferente de zero, podemos escrever

$$
g_{i j}=e_{i}^{a} e_{j}^{b} h_{a b},
$$

onde, $e_{i}^{a}$ são vetores de base com $i=1, \ldots, D ; a=1, \ldots, D$ e $h_{a b}$ é a métrica euclideana, i.e. plana, não degenerada, com assinatura $(+++\ldots+)$ e com o mesmo posto de $g_{i j}$.

Já no caso em que $g_{i j}$ possui determinante nulo, ou seja, sendo uma métrica degenerada, uma forma de definir $g_{i j}$ com posto arbitrário é considerar os vetores de base, assim como a métrica $h_{a b}$, como tendo os indíces a num intervalo numericamente inferior ao dos índices $i$. Neste caso, o posto de $g_{i j}$ é numericamente inferior à dimensão $D$ do espaço. Desta forma iremos considerar uma métrica degenerada conforme veremos na seção a seguir. 


\subsection{A Partícula num Campo de Fundo Gravitacional Degenerado}

Vamos considerar um espaço genérico com dimensão $D$ que possui uma métrica degenerada. Vamos definir a métrica degenerada de dimensäo $D$ e posto r por

$$
g_{i j}=e_{i}^{a} e_{j}^{b} h_{k b}
$$

onde $e_{i}^{a}$ são vetores de base com $i=1, \ldots, D ; a=1, \ldots, r(r<D) ; h_{p a}$ é una métrica euclideana, ie. plana, nạ̃o degenerada e com assinatura $(++\ldots+)$, porém com posto diferente de $g_{i j}$. Noterros também que $e_{i}^{a}$ näo săo matrizes quadradas e assim não possuem inversa.

Desta maneira o determinante de $g_{i j}$ é zero e assim a métrica possuti $D-r$ auto-valores nulos, o que implica em $g_{i j} U_{\alpha}^{j}=0$, sendo $V_{\alpha}^{j}$ os $(D-r)$ auto-vetores associados.

Para uma partícula de massa $m$ movendo-se em ura espaço com métrica degenerada, vamos considerar a lagrangeana como sendo

$$
L=\frac{1}{2} m g_{i j} \dot{x}^{i} \dot{x}^{j}=\frac{1}{2} m \hat{x}^{a} \hat{x}^{b} h_{a b}
$$

onde $\dot{x}^{a}=e_{i}^{a} \dot{x}^{\dot{s}} . O$ momento canonicamente conjugado é entäo

$$
P_{i}=\frac{\partial L}{\partial x^{i}}=m e_{i}^{a} x_{a},
$$

onde $\dot{x}_{a}=h_{a b} \dot{x}^{b}$. Como a métrica é elegenerada, năo podemos inverter está equação para obtermos as velocidades $x^{i}$ em termos dos momentos $P_{i}$. Isto indica a presença de víneulos no sistema. Se considerarmos os $D-r$ auto-vetores $y_{a}^{y}$ aplicados em (2.9), temos

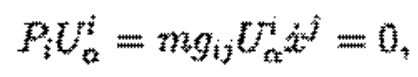

implicando nos vinculos primários

$$
\omega_{\alpha}=p_{i} \omega_{\alpha}^{i}
$$


onde escolhemos $\alpha=q^{*}+1, \ldots, D$ iepresentando $D-r$ componentes.

Podemos achar uma forma explicita para $U_{a}^{\circ}$ a partir das equaços dos momentos (2.9). Nestat equaçôs podemos resolver as velocidades em funça dos momentos apenas para

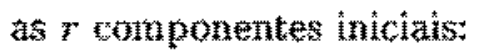

$$
\begin{gathered}
P_{1}=m e_{1}^{a} \dot{x}_{a} \\
\vdots \\
P_{r}=m e_{r}^{a} \dot{x}_{a t},
\end{gathered}
$$

onde obtemos $\hat{x}_{a}=\dot{x}_{a}\left(P_{i^{t}}\right), \quad\left(i^{t}=1, \ldots, r\right)$

As $D-r$ equações restantes,

$$
\begin{gathered}
P_{r+1}=m e_{r+1}^{a} \dot{x}_{a} \\
\vdots \\
P_{D}=m e_{D}^{a} \dot{x}_{a}
\end{gathered}
$$

são yolaços algébricas que formam os vinculos conforme veremos a segurr.

Antes, devemos notar que es possul inversa em um setor reduzido, definida por $e_{a}^{i^{i}}$ com

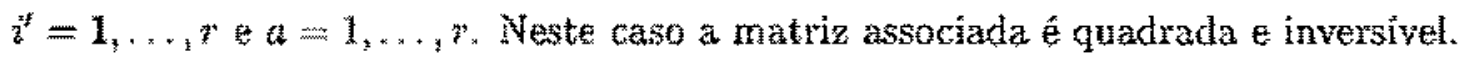

Entäo para

$$
P_{j^{*}}=m e_{i^{*}}^{a} \hat{z}_{a}
$$

onde podemos usat que

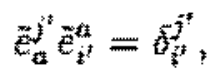

temos

$$
\frac{\vec{e}_{a}^{i^{t}} P_{i^{\prime}}}{m}=\dot{s}_{\alpha}
$$


Considerando os $D-7$ momentos restantes $P_{\alpha}$

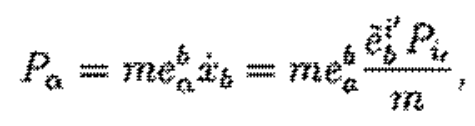

temos os $D-r$ vínculos

$$
\Phi_{a}=P_{\alpha}-e_{\alpha}^{b} e_{z}^{z} P_{z^{*}}=0
$$

Os vínculos, na forme deduzida acima, permitem obter uma forma explicita para $U_{\alpha}^{\mathrm{i}}$ em (2.11),

$$
P_{i} U_{a}^{i}=0=P_{a}-\epsilon_{a}^{b} \vec{e}_{a}^{t} P_{i^{\prime}}
$$

oll seja,

$$
U_{\alpha \alpha}^{i}=\delta_{\alpha}^{i}-e_{a}^{b} e_{b}^{i} \delta_{j^{\prime}}^{i}
$$

A Hamiltoniana sanônica do sistema é dada por

$$
H_{c}=P_{i} \dot{x}^{i}-L=\frac{1}{2 m} \tilde{g}^{i t j^{\prime}} P_{i^{\prime}} P_{j^{\prime}}
$$

com $\bar{g}^{i^{i} j^{\prime}}=\vec{E}_{a}^{i} \bar{e}_{b}^{j^{f}} h^{a b}$ sendo a inversa da métrica não degenerada

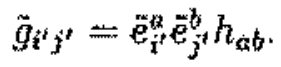

Levando-se em conta os vínculos primários, a Hamiltoniana será

$$
H=H_{\mathrm{c}}+\lambda^{\alpha} \Phi_{\alpha}
$$

onde $\lambda^{a}$ são os multiplicadores de Lagrange associados aos vínculos $\Phi_{\alpha}$.

Contruimos também a álgebra de Poisson dos vínculos:

$$
\begin{aligned}
& \left\{\Phi_{\alpha,} \Phi_{\beta}\right\}=M_{\alpha \beta,} \quad M_{\alpha \beta}=M_{\alpha \beta}^{i} P_{z^{r}}
\end{aligned}
$$

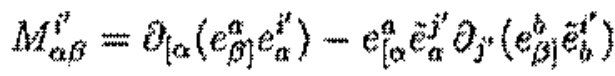

A evoluçào temporal do vínculos, dada por $\hat{\Phi}_{a}=\left\{\Phi_{a}, K\right\}$, como condição de consistễncia, forneç:

$$
\dot{\Phi}_{a}=N_{a}+M_{a \beta} \lambda^{\beta}=0
$$


onde

$$
\begin{aligned}
& N_{\mathrm{a}}=N_{\mathrm{a}}^{i^{t} j^{\prime}} P_{\mathrm{i}^{r}} P_{j^{\prime}}
\end{aligned}
$$

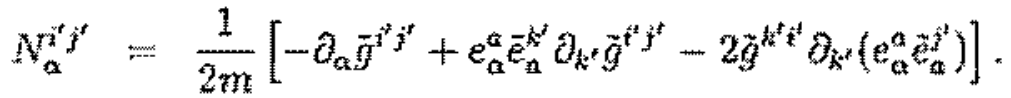

Vamos a seguir considerar os casos en que os vetores de base es sano integráveis ou näo.

\subsection{Vetores de Base Integráveis}

Vamos considerar a situagăo em que $e_{i}^{0}$ é integrável, ou seja, se satisfaz a $Q_{i j} e_{j}^{a}=0$ em todo o espaço.

Neste caso, $M_{\alpha \beta}=0, N_{\alpha}=0$ e $\Phi_{\alpha}=0$, significa entào que $\Phi_{\alpha}$ säo os únicos vínculos do sistema e säo todos de primeira classe.

A simetria local gerada pelos vínculos é dada por

$$
\delta x^{t^{t}}=\epsilon^{\alpha}\left\{\Phi_{\alpha}, x^{i^{4}}\right\}=\epsilon^{\alpha} e_{\alpha}^{b} e_{b}^{j^{i}} \quad \delta x^{\alpha}=-\epsilon^{\alpha}
$$

onde $e^{\circ}$ e parâmetro infinitesimal.

A Lagrangiana do sistema pode tambem ser escrita como

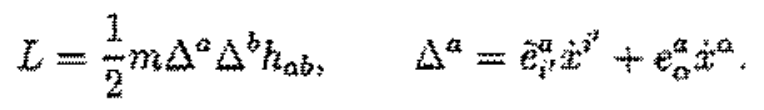

A simatra dada por (2.27), implica em $\delta \Delta^{*}=0$, que faz a lagrangiana ser invariante.

Usando $(2,27)$, todos os $x^{\alpha}$ podem ser fxados por uma escolha de gauge, pois temos $D-r$ vínculos de primeira classe $\Phi_{\alpha}$ e então restam $D-(D-r)=r$ graus de liberdade. Desta forma a Lagrangiana (2.28) passa a ser

$$
L=\frac{1}{2} m \tilde{g}_{i^{1}} j^{i^{i^{t}}} \dot{x}^{i^{t}}
$$


que descreve uma partícula en movimento num espaço com uma métrica năo degenerada $\hat{g}_{i^{t} j^{4}}$ em $r$ dimensöes.

\subsection{Vetores de Base Não Integráveis}

Neste caso $\partial_{[j} e_{j]}^{a} \neq 0$, o que far $M_{a, j} \neq 0$. Entăo a condiçăo de consistência (2.25) precisa ser resolvida para os multiplicadores de Lagrange $\lambda^{a}{ }$ o que envolve a utilização do inverso de $M_{\alpha \beta}$. Porém $M_{\alpha \beta}$ é uma matriz antisaimétrica quadrada de ordem $(D-r)$ e a existência da sua inversa dependerá entäo do valor de $(D-r)$. Devido a isto, existem duas possibilidades, i.e., $D-r$ par ou ímpar.

\subsection{1 $D-r$ Par}

Neste caso $M_{\alpha \beta}$ possui uma inversa $M^{\alpha \beta}$ de tal forma que

$$
\lambda^{a}=-M^{\alpha \beta} N_{\beta}
$$

Todos os multiplicadores de Lagrange $\lambda^{\alpha}$ săo resolvidos. Então, todos os vínculos são de segunda classe. Desta forma o numero dos graus de liberdade será dado por $D-\frac{1}{2}(D-r)=\frac{1}{2}(D+r)$.

Adicionalmente podem ocorrer outras restriçōes na métrica que implicam en $\operatorname{det}\left(M_{\alpha f f}\right)=0$ no caso $D-x$ par. Assim teremos uma variedade de situacöes entre os casos extremos $D$ - p par e impar. Porém vamos considerar apenas estes extremos, o que suficiente pata percebermos como se torna a estrutura dos vínculos do caso com métrica degenerada envolvendo $\partial_{\{1} e_{j}^{a} \neq 0$. 


\subsubsection{D-r İmpar}

Neste caso $\operatorname{det}\left(M_{\alpha a \beta}\right)=0$ e com isto näo podemos resolver (2.25) para todos os $\lambda^{a}, 0$ número de vínculos $\Phi_{\mathbf{a}}$ também é ímpar, significando que existem vínculos de primeira classe entre os $\Phi_{\alpha}$ ou existem novos vínculos. Da mesma forma como analisamos as velocidades em (2.12) e (2.13), 3eguimos um raciocinio similar para estudar as equacônes em (2.25). Visto que $M$ possui inversa para as primeiras $D-r-1$ componentes, vamos resolver as primeiras $D-r-1$ componentes de (2.25) para obtermos as componentes associadas a $\lambda^{\alpha^{\prime}}$ :

$$
\lambda^{B^{t}}=M^{\mathrm{A}^{t} \beta^{t}}\left(N_{n^{t}}+M_{\alpha^{t}, D-\mathrm{r}} \lambda^{D-r}\right)
$$

$\operatorname{com} \alpha^{\prime}, \beta^{\prime}=1, \ldots, D \cdots r-1$, onde devemos notar que rescalamos os índices $\alpha$ para $\alpha^{\prime}$ envolvendo uma contagem efetiva do nümero de componentes utilizadas, ou seja, $(D-r-1)$ componentes.

A componente restante $\dot{\Phi}_{D-\mathrm{r}}$ passa a ser o novo vínculo

$$
\begin{aligned}
& \chi=\dot{\Phi}_{D-F}=N_{D-r}+M_{D-r} z^{2} \lambda^{p^{q}}
\end{aligned}
$$

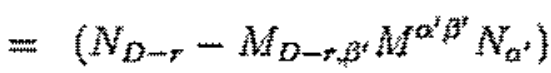

onde utilizamos (2.31).

Devemos notar que como em (2.25) obtemos os vinculos através dos auto-vetores com auto-valores correspondentes nulos, de se esperar que o mesmo aconteça com $\chi$. Podemos entäo definir o auto-vetor de $M$ neste caso, conforme está descrito no apêndice $\mathrm{A}$.

A álgebra de Polsson para os novos vinculos é dada por

$$
\left\{\Phi_{\alpha}, \chi\right\}=\partial_{i^{z}} \Phi_{\alpha} \frac{\partial \chi}{\partial P_{i^{\prime}}}-\frac{\partial \Phi_{\alpha}}{\partial P_{i^{\prime}}} \partial_{i} \chi \chi-\frac{\partial \Phi_{\alpha}}{\partial P_{\beta}} \partial_{\beta} \chi
$$

A Hamiltoniana fica então da seguinte forma

$$
H=H_{t}+\lambda^{\mathbf{a}} \boldsymbol{\Phi}_{\alpha}+\mu \chi
$$


onde $\mu$ é o novo multiplicador de Lagrange associado ao vínculo $\chi$. A partir da Hamiltoniana (2.34), as condiçôes de consistência serão agora dadas por:

$$
\begin{gathered}
\dot{\chi}=\left\{\chi, H_{0}\right\}+\lambda^{\alpha^{\prime}}\left\{\chi, \Phi_{\alpha^{\prime}}\right\}+\lambda^{D-r}\left\{\chi, \Phi_{D-r}\right\}=0, \\
\dot{\Phi}_{\alpha^{\prime}}=N_{\alpha^{\prime}}+\lambda^{\beta^{2}} M_{\alpha^{\prime} \beta^{\prime}}+\lambda^{D-r} M_{\alpha^{\prime}, D-r}+\mu\left\{\Phi_{\alpha^{\prime}}, \chi\right\}=0 \\
\dot{\Phi}_{D-r}=N_{D-r}+\lambda^{\beta^{t}} M_{D-r, \beta^{\prime}}+\mu\left\{\Phi_{D-r_{1}} \chi\right\}=0
\end{gathered}
$$

A partir de (2.36), sabendo que $M$ possui inversa, obtemos entäo $\lambda^{\beta^{t}}$ :

$$
\lambda^{B^{z}}=-M^{\alpha^{\prime} \beta^{\prime}}\left(N_{\alpha^{\prime}}+\lambda^{B-r} M_{\alpha^{r}, D-F}+\mu\left\{\Phi_{\alpha^{i}, \chi}\right\}\right) .
$$

Substituindo $\lambda^{\beta^{t}}$ na equaçäo $(2,37)$, resulta em

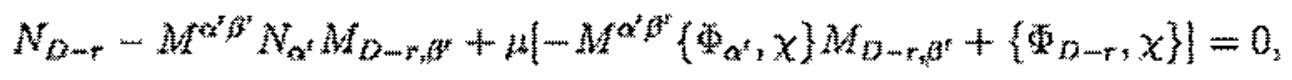

que equivale a

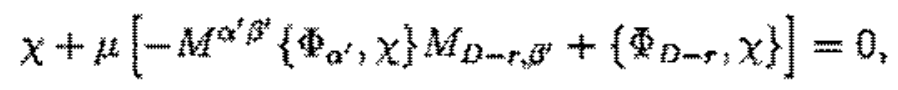

que na superficie dos yinculos implica em $\mu=0$. Além disto, da equasão (2.35) obtemos o valor de $\lambda^{D-x}$;

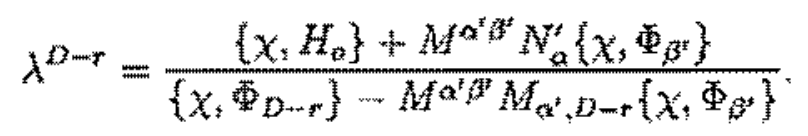

Determinamos entäo todos os multiplicadores de Lagrange, de modo que isto faz com que todos os vínculos sejam de segunda classe Como temos $\left(D-r^{r}+1\right)$ vínculos de segunda classe, 0 espago de fase tem $2 D-(D-r+1)=D+r-1$ dimensôes.

\subsection{Caso $N_{\mu}=0$}

Das equaçóes da evoluçäo de $\dot{\Phi}_{\alpha}$, sabemos que

$$
N_{\alpha}^{i^{t} j^{t}}=\frac{1}{2 m}\left[-\partial_{\alpha} \hat{g}^{i^{t} j^{\prime}}+e_{\alpha k}^{a} e_{\alpha}^{k^{x}} \partial_{k^{\prime}} \ddot{g}^{i j^{t}}-2 \tilde{g}^{k^{\prime} i^{t}} \partial_{k^{\prime}}\left(e_{\alpha}^{a} \bar{e}_{\alpha}^{j^{t}}\right)\right]
$$


Entretanto, podenos escrever $\mathrm{N}_{0}^{t^{*} j^{t}}$ em termos dos vetores de base, ou seja,

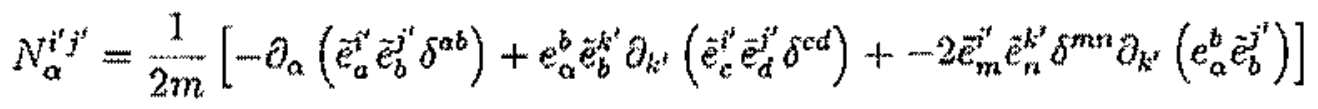

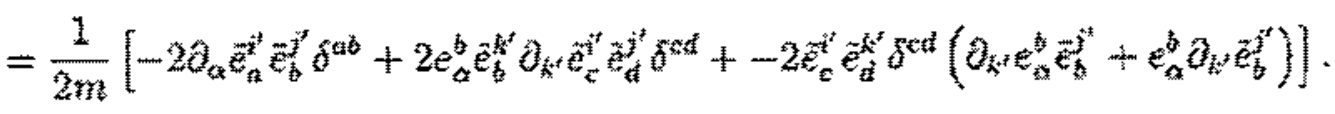

Usando que

$$
\begin{aligned}
& \partial_{\alpha} \vec{e}_{a}^{i t}=-\vec{e}_{a}^{k^{\prime}} e_{c}^{i} \partial_{\alpha} \tilde{e}_{k^{\prime}}^{c}
\end{aligned}
$$

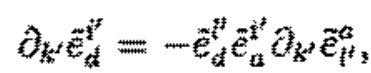

obtemos

$$
N_{\alpha}^{i^{\prime} j^{t}}=\tilde{e}_{\alpha}^{k^{\prime}} \hat{e}_{c}^{i^{\prime}} \hat{e}_{b}^{j^{\prime}} \delta^{a b}\left(\partial_{[\alpha} \hat{e}_{\left.k^{\prime}\right]}^{c}-f_{\alpha}^{l} \partial_{\left[l^{\prime}\right.} \tilde{e}_{\left.k^{\prime}\right]}^{c}\right)
$$

onde

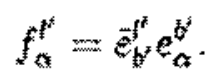

Logo,

$$
N_{\alpha}^{i^{\prime} j^{\prime}} P_{i^{\prime}} P_{j^{\prime}}=N_{\alpha}=\left(\partial_{[\alpha} e_{\left.k^{\prime}\right]}^{c}-f_{\alpha}^{l^{\prime}} \partial_{\left[l^{\prime}\right.} e_{\left.k^{\prime}\right]}^{e}\right) \tilde{e}_{\alpha}^{N^{\prime}} \delta^{a b} \tilde{P}_{b} \tilde{P}_{c}
$$

onde $\tilde{P}_{b}=e_{b}^{i^{i}} P_{i}$.

Podernos entäa concluir que se os vetores de base satisfaztm a

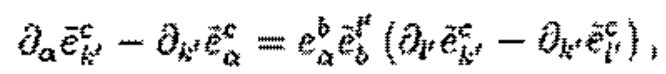

temos $N_{a}=0$. Neste caso, veremos a seguir como ficará $M_{\alpha \beta}$. Substituindo (2.50) nas componentes de $M_{\alpha B}$, temos que

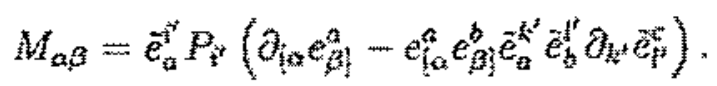


Entäo, $N_{a}=0$, com as condiçōes (2.50), implicam en

$$
M_{\alpha \beta}=e_{\alpha}^{i^{\prime}} P_{i^{\prime}}\left(\partial_{i \alpha} e_{\beta !}^{a}-f_{[\alpha}^{k^{\prime}} f_{\beta !}^{l^{\prime}} \partial_{k^{*}} \tilde{e}_{i^{*}}^{c}\right)
$$

Podemos notar que mesmo com $\partial_{i i} e_{j !}^{a} \neq 0$, a partir das relarōes acima, ainda podemos ter $N_{\alpha}=0=M_{\alpha \beta \beta}$, caso que ocorre diretamente quando $\partial_{[i} e_{j]}^{a}=0$.

A relação entre $N_{\alpha}=0=M_{\alpha}$ com $\partial_{i j} e_{j !}^{a} \neq 0$ ainda poderá revelar propriedades geométricas que relacionam os casos integrável e não integrável. Išto sera tratado em urn futuro projeto.

Vamos analisar o caso em que $N_{\alpha}=0$ e $M_{\alpha \beta} \neq 0$. Neste caso,

$$
\dot{\Phi}_{x}=M_{\alpha x p} \lambda^{\beta}=0
$$

Para $D-m$ par existe a inversa $M^{a j}$. Então,

$$
\lambda^{\beta} M_{\mathrm{n} \beta} M^{\alpha \rho}=0
$$

0. que resulta $x^{p}=0$

Para $D-r$ ímpar não existe a inversa $M^{a}{ }^{\beta}$. Mas

$$
M_{\alpha \beta} \lambda^{\beta}=\lambda^{\beta^{t}} M_{\alpha \beta^{\prime}}+\lambda^{D-r} M_{\alpha D-r}=0
$$

ou ainda,

$$
\begin{aligned}
\lambda^{\beta^{\prime}} M_{a^{\prime} \beta^{\prime}}+\lambda^{D-r} M_{a^{\prime} D-r} & =0 \\
\lambda^{\beta^{r}} M_{D-r \beta^{\prime}} & =0 .
\end{aligned}
$$

Segue que

$$
\lambda^{\beta^{\prime}}=-\lambda^{D-\mathrm{r}} M_{\alpha^{\prime} D-r} M^{\alpha^{t} B^{r}}
$$

ficando $A^{D-r}$ indeterminado. 
Mas $\left\{\phi_{\alpha}, \phi_{\beta}\right\} \neq 0$, o que implica num conjunto com um número ímpar de vínculos de segunda classe. Neśte caso existirá uma combinação dos vínculos que será de primeira classe.

Se considerarmos as combinaŗōes

$$
\Phi_{p^{\prime}}=0 \quad \Gamma=\Phi_{D-F}-M_{\alpha^{2} D \cdots} M^{\alpha^{\prime} \beta^{t}} \Phi_{\beta^{\prime}}=0
$$

verificamos que

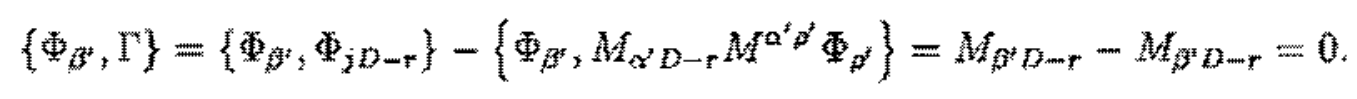

Analogamente $\left\{\right.$ c $\left._{D-n} \Gamma\right\}=0$. Então temos que $\Phi_{\beta^{r}}$ são de segunda classe e $\Gamma$ é de primeira classe. Podemos verificar ainda que $\dot{\Gamma}=0=\dot{\Phi}_{\alpha^{*}}$.

Entäo conclúnos que para o caso em que $N_{a}=0$ e $M_{\alpha \beta} \neq 0$ temos, para $D-r$ impar, $D-r-1$ vinculos de segunda classe e um vínculo de primeira classe de tal forma que o sistema terâ $D+r-1$ graus de liberdade no espaço de fase. Para $D-r$ par há $D-r$ vínculos de segunda classe implicando em um sístema com $D+r$ grats de liberdade no espaço de fase.

\subsection{Isometrias e Vínculos de Primeira Classe com Métricas Degeneradas}

Uma isometria e defintda como un automorfismo do uma variedade, sendo assim, uma transformaçăo que preserva a métrica da variedade. A derivada de Lie da métrica $g_{i j}$ com respeito ao vetor de Killing $K_{a}$ é que representa a isonetria [24]. Em componentes, isto significa

$$
\left(\mathcal{L}_{K_{n}} g\right)_{i j}=K_{\alpha}^{m} \partial_{m} g_{j j}+\left(\partial_{i} K_{\alpha}^{m}\right) g_{m j}+\left(\partial_{j} K_{\alpha}^{m}\right) g_{m}=0
$$

onde $\alpha=1, \ldots, r$ é $o$ indice associado a número de vetores de Killing existentes para uma determinada isometria; $m$ é o indice das componentes de um vetor $K_{\alpha}$. 
Para a Lagrangiana da partícula nào relativística nura fundo gravitacional, escrita em (2.8): consideremos a seguinte transformaçăo:

$$
\delta x^{i}=-\varepsilon^{\alpha} K_{\alpha}^{i}
$$

onde $\epsilon^{\alpha}$ é um parầmetro infinitesimal constante.

Com (2.61), vejamos como a Lagrangiana en (2.8) se transforma, ou seja,

$$
\delta L=m\left(\frac{1}{2} \delta g_{i j} \dot{x}^{i} x^{j}+g_{j j} \dot{x}^{i} \delta x^{j}\right)
$$

onde temos

$$
\begin{aligned}
& \delta g_{i j}=\frac{\partial y_{i j}}{\partial x^{\prime}} \delta \\
& =-a_{4} e^{\alpha} K_{x} \\
& \delta \dot{x}^{i}=-\left(\epsilon^{\alpha} K_{\alpha}^{\mathrm{i}}\right) \\
& =-\epsilon^{\alpha} \partial_{j} K_{\alpha}^{i} \dot{x}^{j}-\varepsilon^{\alpha} K_{\alpha}^{i} .
\end{aligned}
$$

Substituindo (2.63) e (2.64) em (2.62), temos

$$
\begin{aligned}
& \delta L=-m \epsilon^{\alpha}\left(\frac{1}{2} K_{\alpha}^{i} \partial_{i} g_{i j} \dot{x}^{i} \dot{x}^{j}+g_{i j} \partial_{k} K_{\alpha}^{j} \dot{x}^{i} \dot{x}^{j}\right)-m \dot{\epsilon}^{\alpha} K_{\alpha}^{k} g_{j} \dot{x}^{j} \\
& =-\frac{1}{2} m \varepsilon^{\alpha}\left(K_{\alpha}^{i} \partial_{i} g_{i j} \dot{x}^{i} \dot{x}^{j}+\partial_{l} K_{\alpha}^{j} g_{i j} \dot{x}^{i} \dot{x}^{j}+\partial_{k} K_{\alpha}^{i} g_{i j} \dot{x}^{j} \dot{x}^{j}\right)-m \dot{\epsilon}^{\alpha} K_{\alpha}^{i} g_{i j} \dot{x}^{j} \\
& =-\frac{1}{2} m \epsilon^{\alpha}\left(K_{\alpha}^{b} \partial_{i j} g_{i j}+g_{j} \partial_{i} K_{a}^{l}+g_{i t} \partial_{j} K_{a}^{j}\right) \dot{x}^{i} \dot{x}^{j}-m \epsilon^{\alpha} K_{\alpha}^{i} g_{j} \dot{x}^{j}
\end{aligned}
$$

Entăo, para a transformaçăo (2.61), a Lagrangiana é invariante desde que a métrica satisfaça ì condiçào de isometria (2.60) e o parâmetro f seja constante.

Devemos notar que tanto para a definiçăo de isometria en (2.60) como para a transformação infinitesimal efetuada na Lagrangiana näo foi necessário o inverso de $g_{i j}$. Com isto, podemos analisar tais operaçōes no caso em que a métrica é degenerada. 
Neste caso, vamos considerar a métrica definida em (2.7) sabendo que esta possú $D-r$ auto-vetores nulos $U_{a}^{i}$ que implicarăo em $D-r$ vínculos $\Phi_{\alpha}=0$. Conforme dernonstrado na Seçäo 2.2 do Capítulo 2 , sabemos que quando os vetores de base satisfazem a $\partial_{[i} e_{j]}^{a}=0$, os vínculos $\Phi_{\alpha}=0$ são de primeira classe. Os vínculos sendo de primeira classe geram as transformaçôes (2.27) que deixam a Lagrangiana na forma (2.28) invariante. Entretanto, 2o considerarmos as transformaçōes (2.27) reescritas como

$$
\hat{d} x^{i}=-\epsilon^{\alpha}(t) U_{\alpha}^{i}
$$

tenos que a Lagrangiana na forma (2.8) se transforma proporcionalmente a

$$
U_{\alpha}^{m} \partial_{m} g_{i j}+\left(\partial_{i} U_{\alpha}^{m}\right) g_{m j}+\left(\partial_{j} U_{\alpha}^{m}\right) g_{i m s}
$$

que ao comparamos com $(2.60)$, corresponde a $\left(\mathcal{C}_{V_{\alpha}} g\right)_{; j}$. Com isto, temos que a Lagrangiana é invariante se ocorrer uma isometria para a métrica degenerada. Nesta isometria os auto-yetores nulas correspondem aos vetores de Kïlling.

Devemos notar que para a ação com métrica degeneradas existe uma caracteristica especial,i.e., a transformaçăo infinitesimal (2.66) já é local, ou seja, o parânetroto $\epsilon^{\circ}$ depende do tempo, ao contrârio de uma métrica que não é degenerada. Esta localidade é devido a transformação (2.66) ser gerada por vínculos de primeira classe. Para o caso em que a métrica não é degenerada, não existem vínculos primários e nem os de primeira classe porque näo hả auto-vetores nulos para esta métrica. Assim, para haver uma isometria, no caso de uma métrica não degenerada, que pernita a invariância da Lagrangiana, é necessário que o parâmetro $\epsilon^{\text {a }}$ seja constante a derivada de Lie seja considerada com vetores de hilling especificos de acordo con a geometria do espaço. Neste caso a condição de isometria $\bar{t}$ a usual, ou seja, é considerada como o anulamento da derivada covariantét simetrizada do vetor de Killing [25]. 


\section{Capítulo 3}

\section{A Partícula Relativística}

\section{Introdução}

Uma particula relativistica massiva movendo-se num espaço-tempo de Minkowski com $D$ dimensóes é descrita por una açầo proporcional ao comprimento da trajetónia da partícula ao longo da linha de universo [26]. Ao considerarmos o movimento em um espaçonterripo curvo de $D$ dimensōes com métrica $g_{\mu \nu}$ e determinante diferente de sero apresentaremos, na Seção 3.1, a formulação Hamiltoniana, cuja álgebra dos vínculos é de primeira classe.

Considerando que a açäo e as equações de movimento na Seçăo 3.1 estāo bem definidas mesmo no caso em que o determinante de $g_{\mu \nu}$ é nulo, ou seja, sem admitir a existência do inverso da mêtrica, construímos na Seção 3.2 o formalismo Hamiltoniano. Neste caso, encontramos vinculos adicionais que serão de primeira ou segunda classe, dependendo explicitamente da métrica. Apresentamos para cada forma da métrica a classificajão dos vínculos a a dinâmica efetiva que descreve o movimento da partícula. 


\subsection{A Partícula num Campo de Fundo Gravitacional não Degenerado}

A açäo de uma partícula relativística de massa $m$, movendo-se em um espaço-tempo curwo descrito por uma métrica $g_{\mu \nu}$ é dada por:

$$
S=\int_{n_{1}}^{n} d r\left(\frac{1}{2 e} a_{n i b} \hat{x}^{*} \dot{x}^{\nu}-\frac{e}{2} m^{2}\right)
$$

onde $r$ t o parämetro de evolucăo e e uma variaval auxiliar que é conhecida como einbein.

As equaçöe de movimento obtidas a partir de (3.1) são dadas por:

$$
\begin{aligned}
& \frac{\partial S}{\partial e}=\frac{1}{2 e^{2}} g_{a t} \dot{a}^{* t} z^{* t}+\frac{m^{2}}{2}=0
\end{aligned}
$$

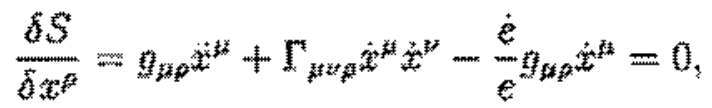

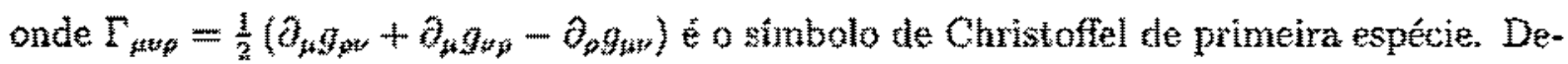
vemos notar que a partir de (3.2) podemos obter forma usual da ajäo que é proporcional ao comprimento da trajetoria da partcula. Con isto substibimos obtido de (3.2) em $(3.1)$ e obtemos

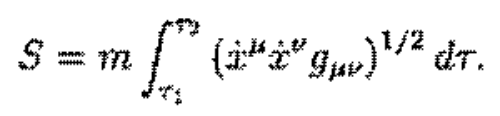

A formulaçäo en (3.4) é importante como uma motivaçăo básica para definirmos, conforme veremos no capitulo seguinte, a açă de uma corda, uma vez que uma corda movendose no espaço-tempo gera uma superfície. Entretanto, a formulação da ação em (3.1) em termos da variável auxiliar e permite considerar o limite de massa zero.

Outro aspecto importante é que $(3.1),(3.2)$ e (3.3) estăo bem definidas mesmo se a métrica năo possuir inversa. 
Vamos considerar os momentos canonicamente conjugados a $e$ e $x^{*}$ respectivamente dados por

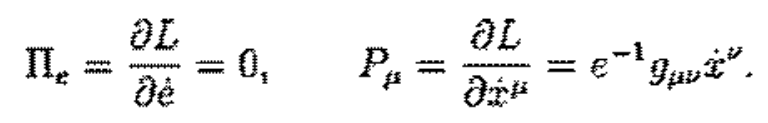

Considerando que $g_{z y}$ admite inversa, construmos a Hamiltoniana canônica, que é dada por

$$
H_{k}=\frac{e}{2}\left(P_{\mu} P_{u} g^{\mu \nu}+m^{2}\right)
$$

Como o momento associado a e näo é proporcional à velocidade, temos a existência de un vinculo $\Pi_{t}=0$ no sistema. Considerando a evolução deste vínculo, obtemos

$$
\dot{\mathrm{I}}_{e}=\frac{e}{2}\left(P^{2}+m^{2}\right)=0
$$

onde $P^{2}=P_{\mu} P_{\nu} g^{\mu \nu}$, que implica na existência do vínculo

$$
\varphi=p^{2}+n^{2}=0
$$

Como $\left\{\hat{\varphi}, \Pi_{e}\right\}=0$, então os vínculos $\varphi=0$ e $\Pi_{g}=0$ são de primeira classe e a Hamiltoniana canônica é diretamente proporcional a $\varphi$. Esta proporcionalidade é uma caracteristica das teorias invariantes por reparametrizaçầ, sendo o vinculo de primeira clazse o gerador da simetria.

Consideremos a seguir quais as propriedades que irảo surgir no caso em que gyt não adinite inversa. 


\subsection{A Partícula em Campo de Fundo Gravitacional Degenerado}

Na swân anterior, observamos que para obternos as yelecidades associadas a $x^{*}$ ne cessitamos do inverso de $g_{y}$. Assim como no caso da particula nảo relativisticta, o fato

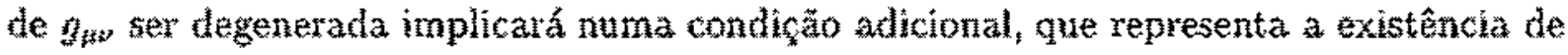
un novo vínculo primário.

Utilizando a mesma açäo, equaçöes de movimento e momentos, considerados na seçäo anterior, vamos construir a Hamiltoniana canônica para o caso em que $g_{\mu}$ é degenerada.

Vamos considerar novamente a métrica na seguinte forma:

$$
g_{\mu \nu}=e_{\mu}^{a^{2}} \mathrm{e}_{b}^{b^{z}} \eta_{a^{\prime} b^{\prime}}, \quad \eta_{a^{\prime} b^{\prime}}=\operatorname{diag}(-1,+1,+1, \ldots,+1)
$$

tal que $\mu=0,1, \ldots, D-1 \quad a^{t}=0,1, \ldots, r-1$. Verificamos facilmente que o determinante da métrica se anula.

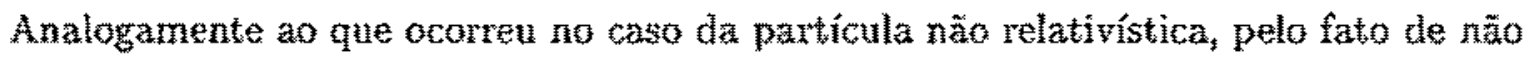
podermos obter todos os t $^{*}$ a partir de $p_{1}$ em $(3.5)$, temos um vinculo primáno adicional 帮 de tal forma que os vínculos primários säo

$$
\begin{aligned}
& \Pi_{e}=0, \\
& \Phi_{\underline{\underline{u}}}=p_{\underline{\underline{u}}}-e_{\underline{\underline{\mu}}}^{a^{\prime}} \hat{e}_{a^{\prime}}^{u^{\prime}} P_{k^{t}}=0
\end{aligned}
$$

onde $\mu^{\prime}=0,1, \ldots, r^{*}-1 ; \quad \underline{\mu}=\gamma_{3}^{*} \ldots, D-1$.

Considerando $(3,11)$ e utilizando a seguinte relatâno entre os vetores de base

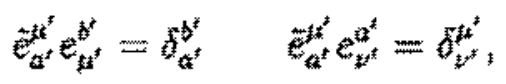

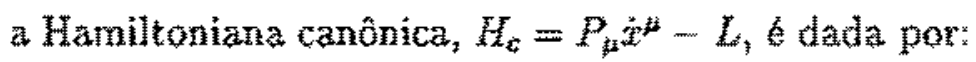

$$
H_{c}=\frac{c}{2}\left(p^{2}+m^{2}\right)
$$


onde,

$$
\begin{aligned}
\bar{P}^{2} & =\ddot{g}^{u^{\prime} t^{\prime}} P_{u^{\prime}} P_{v^{\prime}}, \\
\bar{g}^{\mu^{\prime} w^{\prime}} & =\eta^{a^{\prime} b^{\prime}} \vec{e}_{a^{\prime}}^{u^{\prime}} e_{b^{\prime}}^{y^{\prime}}
\end{aligned}
$$

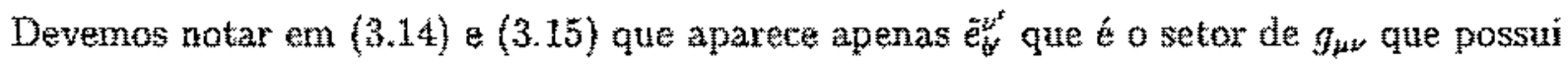
inversa. Neste setor os indices estào definitos por $t^{t}=0,1, \ldots,+-1$ c $a^{*}=0,1, \ldots,-1$ de tal forma que $e_{a^{*}}^{\text {ta }^{*}}$ se torra uma matriz quadrada que admite inversa.

Considerando os vínculos primários obtidos em (3.10) e (3.11), temos a Hamiltoniana primária

$$
H_{p}=\frac{e}{2}\left(\hat{p}^{2}+m^{2}\right)+\lambda \Pi_{2}+\lambda \Phi^{\underline{\mu}}
$$

onde $\lambda$ e $\lambda$ a s

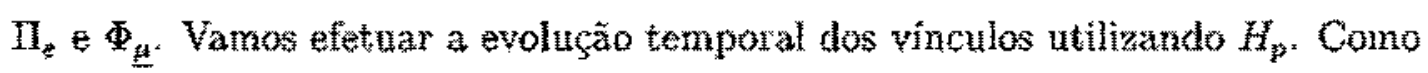

$$
\left\{e, \Pi_{e}\right\}=1, \quad\left\{x^{\mu}, P_{u}\right\}=\delta_{\nu}^{\mu}
$$

obtemos que

$$
\begin{aligned}
& \mathrm{n}_{2}=-\frac{1}{2}\left(\tilde{p}^{2}+m^{2}\right) \equiv t=0
\end{aligned}
$$

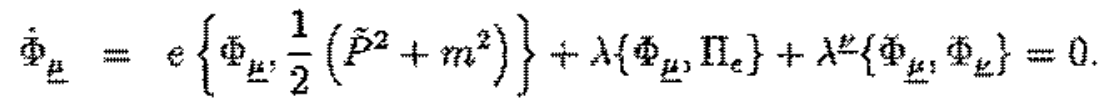

Devemos observar que a evoluçao de $\Pi_{s}$ resulta em um vínculo $\varphi=\left(\vec{P}^{2}+m^{2}\right)=0$ que se assemelha ao vínculo $\varphi$ em (3.8) do caso năo degenerado. Entretanto, no caso degenerado, $\tilde{P}^{2}$ é construído em apenas um setor de $g_{p w}$ conforme podemos notar em $(3.14)$.

Calculemos a seguir os parênteses de Poisson entre os vinculos. Am (3.18) temos

$$
\begin{aligned}
& \left\{\tilde{\Phi}_{\underline{\mu}}, \frac{1}{2}\left(\tilde{p}^{2}+m^{2}\right)\right\}=\frac{1}{2}\left[-\partial_{\underline{g}} \tilde{g}^{\dot{\rho}^{\prime} \lambda^{\prime}} P_{p^{\prime}} P_{\lambda^{\prime}}\right.
\end{aligned}
$$

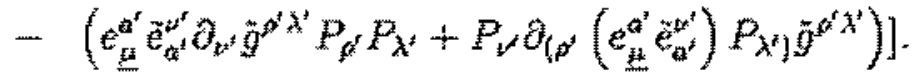


Vamos rescrever (3.19) considerando (3.12) e

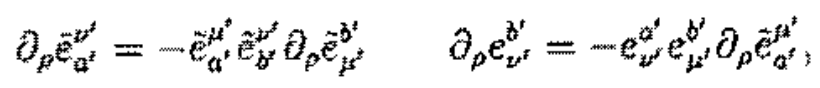

onde (3.20) é obtida a partir de (3.12). Com isto passamos a ter

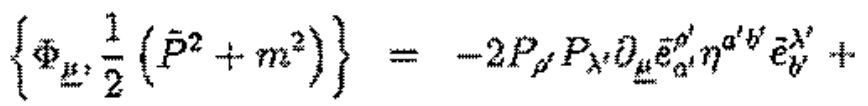

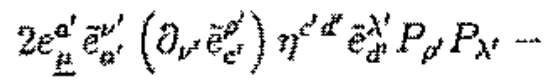

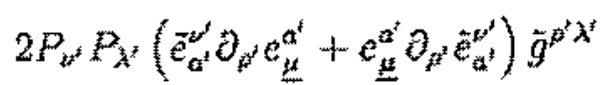

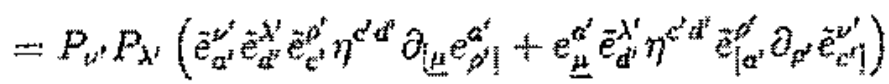

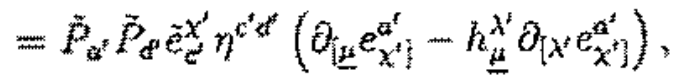

onde

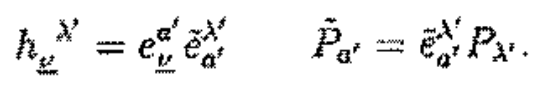

Consideremos agora

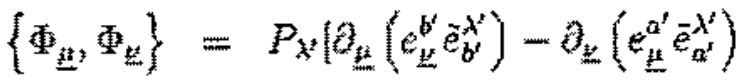

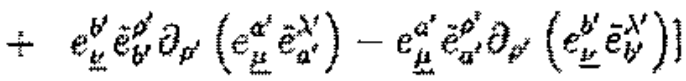

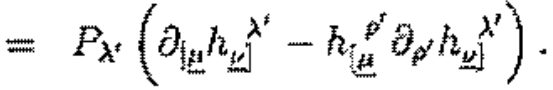

Entảo, podemos escrever (3.18) como:

$$
\dot{\Phi}_{\underline{\mu}}=N_{\underline{\underline{\mu}}}^{*}+\lambda^{\underline{\underline{u}}} M_{\underline{\mu L}}=0
$$

onde 


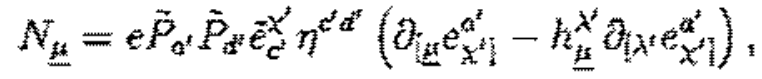

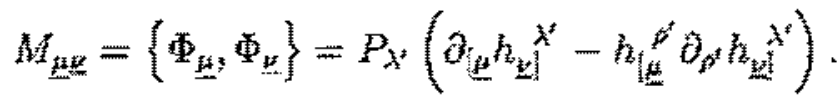

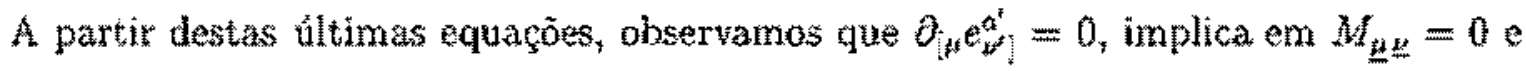
$N_{j}=0$ e analogamente ao caso da partícula năo relativística, näo há novos vínculos, o que torna todos os vínculos presentes como sendo de primeira classe. Neste caso, existem $r-1$ graus de liberdade no espaço de configuração.

Porém, considerando que $M_{\underline{\mu}} \neq 0$ e $N_{\mu} \neq 0$ temos os seguintes casos a considerar:

\subsubsection{Caso $D-r$ Par}

Para $D-r$ par, sabemos que existe a inversa de $M_{\underline{\mu} \underline{\underline{ }}}$ que representaremos por $(M) \underline{\underline{\mu}}$, Entăo, de (3.26) obtemos

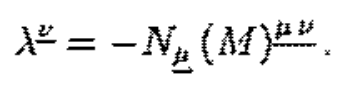

Mas

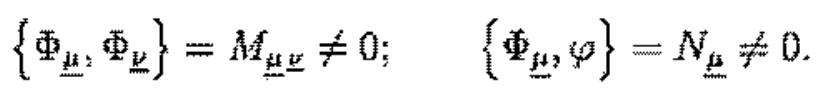

Entâo, existem $D-r+1$ vínculos de segunda classe, ou seja, um número ímpar destes vínculos. Para termos um número par de vínculos de segunda classe será necessário extrair uma combinação linear dos vínculos que seja de primeira classe. Vamos escrever essa combinaçäo linear como

$$
\phi=f \varphi+f^{\mu} \phi_{\mu}
$$

Assumindo que $\phi$ é de primeira classe, obtemos 


$$
\begin{aligned}
\{\phi, \varphi\} & =f^{\underline{\mu}}\left\{\phi_{\underline{\mu}}, \varphi\right\}=\frac{2}{e} f \underline{\underline{\mu}} N_{\underline{\mu}}=0, \\
\left\{\phi, \Phi_{\underline{\rho}}\right\} & =f\left\{\varphi, \Phi_{\underline{\rho}}\right\}+f \underline{\mu}\left\{\underline{\Phi}_{\underline{\mu}}, \Phi_{\underline{\rho}}\right\} \\
& =\frac{-2}{e} f N_{\underline{\rho}}+f \underline{\mu}_{\underline{\mu} \underline{\rho}}=0 .
\end{aligned}
$$

Uma soluçāo para (3.32) é dada por

$$
f^{\underline{\mu}}=N_{\underline{\rho}} M \underline{\mu \rho},
$$

uma vez que $M \underline{\mu \underline{\rho}} N_{\underline{\rho}} N_{\underline{\mu}}=0$ devido à antissimetria de $M \underline{\mu \underline{\rho}}$.

Substituindo (3.34) em (3.33), temos

$$
f \frac{2}{e} N_{\underline{\rho}}=N_{\underline{\nu}} M \underline{\mu \underline{\mu}} M_{\underline{\mu} \underline{\rho}}=N_{\underline{\nu}} \delta \underline{\underline{\rho}}
$$

que resulta em $f=\frac{e}{2}$. Logo, o vínculo de primeira classe que envolve a combinação dos vínculos iniciais, é dado por:

$$
\phi=\frac{e}{2} \varphi+M \underline{\mu \rho} N_{\underline{\mu}} \Phi_{\underline{\mu}}
$$

Desta forma existem $(D-r)$ vínculos de segunda classe $\Phi_{\underline{\mu}}$ e dois vínculos de primeira classe $\phi$ e $\Pi_{e}$. Entāo existem $D+r-2$ graus de liberdade no espaço de fase.

\subsubsection{Caso $D-r$ Ímpar}

Para $D-r$ ímpar não existe inversa de $M_{\underline{\mu} \underline{\nu}}$. Então, seja $\underline{\mu}^{\prime}=1, \ldots, D-r-1$ de tal forma que $\underline{\mu}=\left(\underline{\mu^{\prime}}, \underline{D-r}\right)$. Neste caso, a partir de (3.26), obtemos

$$
N_{\underline{\mu^{\prime}}}+M_{\underline{\mu^{\prime}} \underline{u}^{\prime}} \lambda^{\underline{\underline{u}}^{\prime}}+M_{\underline{\mu^{\prime}} \underline{D-r}} \lambda \stackrel{D-r}{\underline{D-r}}=0=\dot{\Phi}_{\underline{\mu^{\prime}}}
$$




$$
N_{D-r}+M_{D-r e^{P E}}=0=\underline{\Phi}_{\underline{D-r}}
$$

Então

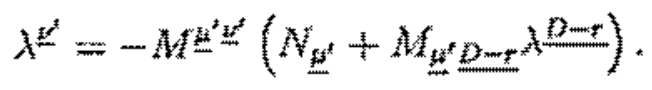

Substituindo (3.39) en (3.38), temos

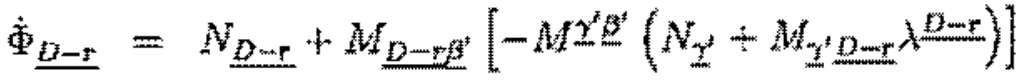

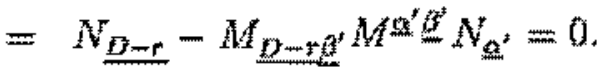

Verificamos que a expressào de $\dot{\Phi}_{D \rightarrow r}$, através da substitufyào de $\lambda^{\prime \prime}$ encontrado, envolve uma combinação de $N$ e $M$. De fato encontramos um novo vínculo dado por

$$
\chi=N_{\underline{D-r}}-M_{D-r g^{\prime}} M^{\alpha^{\prime} \underline{B}^{\prime}} N_{\underline{\alpha}^{\prime}}=0
$$

Vejamos como fica a evoluçäo dos novos vínculos:

$$
\begin{aligned}
& \dot{\chi}=\frac{e}{2}\{\chi, \varphi\}+\lambda \stackrel{\mu^{\prime}}{ }\left\{x, \Phi_{\underline{H}^{\prime}}\right\}+\lambda \stackrel{\lambda-r}{-}\left\{x, \Phi_{D-r}\right\} \\
& =\frac{e}{2}\{x, \phi\}-M^{2} H^{2} N_{e}\left\{x, \omega_{\mu^{t}}\right\}
\end{aligned}
$$

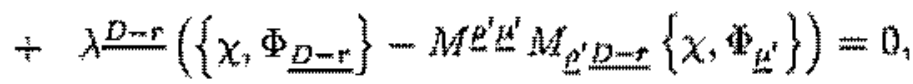

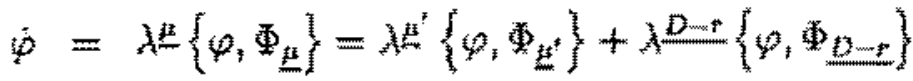

$$
\begin{aligned}
& =-\frac{2}{e}\left[\lambda z^{3} N_{E^{3}}+\lambda \stackrel{D-P}{ } N_{D-g}\right]
\end{aligned}
$$

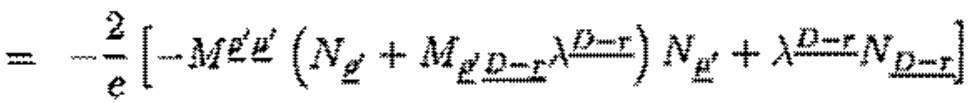

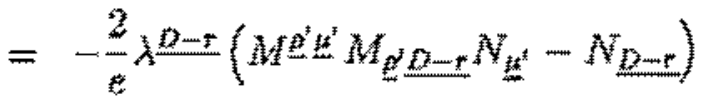

$$
\begin{aligned}
& =-\frac{2}{\mathrm{e}} \lambda \stackrel{b-x}{\underline{b}} x=0 \text {. }
\end{aligned}
$$


Da evoluçầo de ă năo obtemos novos vínculus. Da evoluçăo de $\chi$ obtemos

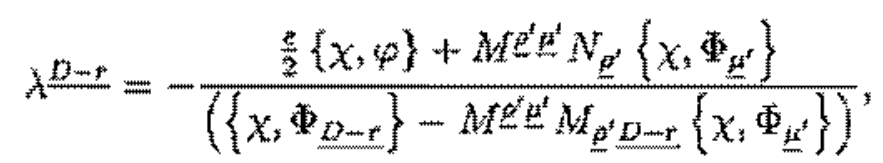

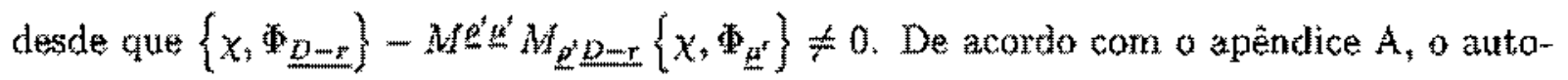
vetor nulo de $M_{\text {北世 }}$ é dado por

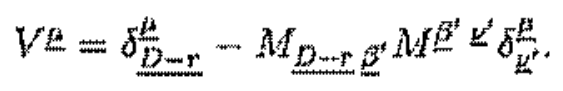

Porérm,

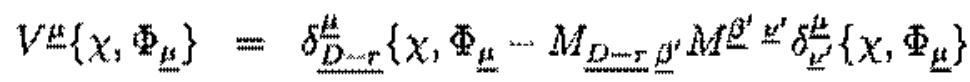

$$
\begin{aligned}
& =\left\{x, \underline{\Phi_{D-r}}\right\}-M_{D-r} \underline{z^{\prime}} M \underline{\underline{B}}^{\underline{L}^{\prime}}\left\{x, \Phi_{L^{3}}\right\}
\end{aligned}
$$

que corresponde ao denominador da expressão de $\lambda \stackrel{D-r}{\operatorname{em}}$ (3.44). Então,

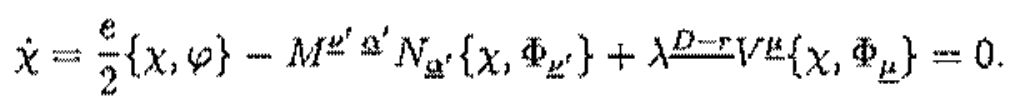

Devemos observar que

$$
\begin{aligned}
& V k\left\{\varphi, w_{u}\right\}=\frac{-2}{e} V N_{\underline{u}}=0,
\end{aligned}
$$

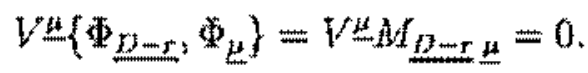

Porém sabemos que $\chi=\Phi_{\underline{D}-r} \neq \Phi_{D-r}$, que comparando com (3.49), nos permite conduir que $V$ u $\left\{X, \Phi_{H}\right\} \neq 0$. Entäo $(3.47)$ permite determinar

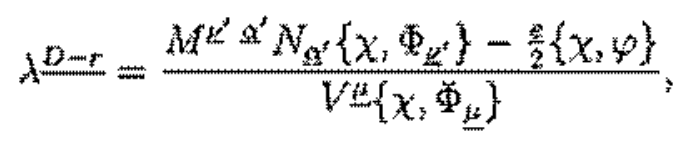

onde o denominador nâo se anula. Logo, determinamos $\lambda \underline{x} \lambda \underline{D-5}$. Isto implica que os vínculos $\Phi_{\underline{z}}$ sâo de segunda classe. 
Vamos agora deterninar se $\chi$ é de segunda classe. Isto será determinado de forma anăloga àquela apresentada no caso nảo relativistico, com a diferença que no presente caso existe um vínculo adicional $\%$. A Hamiltoniana ể dada por

$$
H=\frac{e}{2} \varphi+\lambda \underline{\underline{\Phi_{\underline{L}}}}+\mu \chi
$$

Vamos verificar se a evolução dos vínculos pernite determinar o multiplicador de Lagrange th associado ao vínculo $x$.

$$
\begin{aligned}
& \dot{\varphi}=\lambda \mu\left\{\varphi, \Phi_{\mu}\right\}+\mu\{\varphi, \chi\} \\
& =\frac{-2}{e} \lambda t^{2} N_{z^{2}}-\frac{2}{e} \lambda{ }^{D}+N_{D-r}+k\{\{, x\}=0, \\
& \dot{x}=\frac{e}{2}\{x, \varphi\}+\lambda \mu\left\{x, w_{1}\right\}=0, \\
& \dot{\Phi}_{\underline{\mu}}=N_{\underline{\mu}}+\lambda \underline{\underline{\mu}}\left\{\Phi_{\underline{\mu}}, \Phi_{\underline{\underline{\mu}}}\right\}+\mu\left\{\Phi_{\underline{\underline{\mu}}}, \chi\right\}=0 .
\end{aligned}
$$

Considerando (3.54) com $\underline{\mu}=\underline{\mu}^{\prime}, \underline{D}-r$, temas

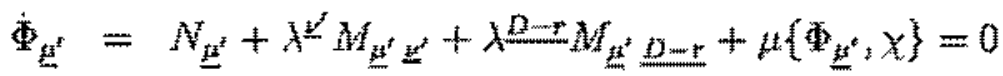

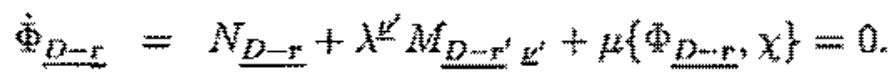

De $(3.55)$ obtemos

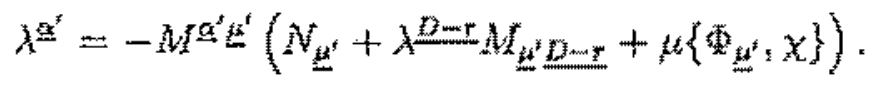

Substituindo (3.57) em (3.56), obtemos

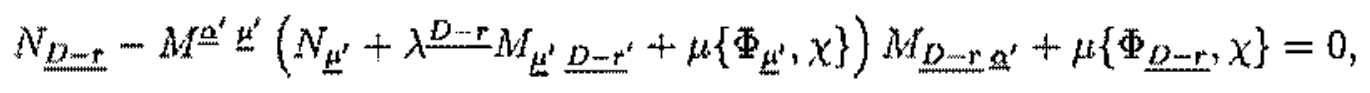


que é equivalente a

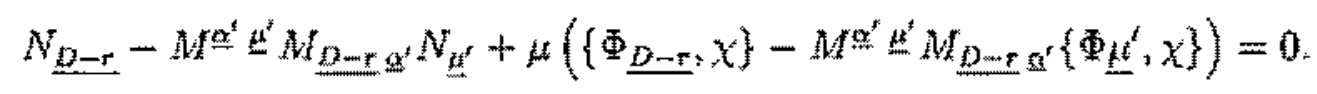

Isto resulta em

$$
\left.x-u\left(v+x, w_{y}\right\}\right)=0
$$

De $(3.60)$ conduímos, entäo, que $\mu=0$.

Entāo em (3.52) temos:

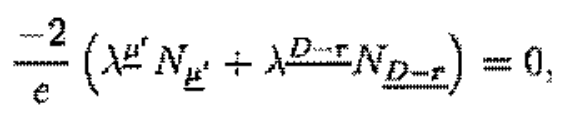

que equivale a

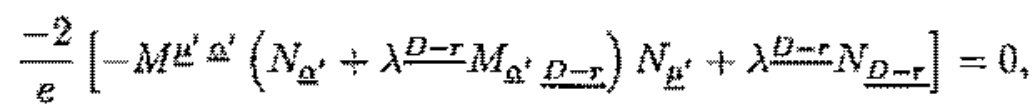

resultando em

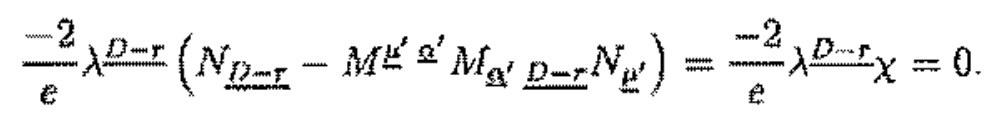

Com esta ultima equaçoconeluimos que $\dot{\varphi}=0$ näo gera novos vínculos. Fm $(3.53)$ temos

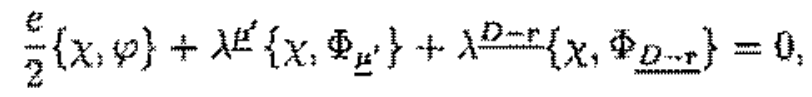

que equivale a

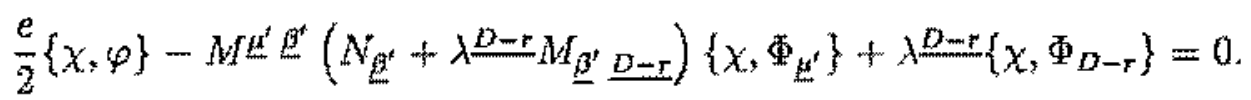

Esta ultima implica no valor de $\lambda^{D-r}$ já encontrado anteriormente em $(3.44)$ ou ata forma equivalente em $(3.50)$.

Determinamos entăo todos os multiplicadores de Lagrange associados anc vinculos $x \mathrm{e} \Phi_{\underline{\mu}}$, o que implica que estes são vinculos de segunda classe. Porem, sabemos que $\left\{\varphi, \Phi_{\underline{y}}\right\}=-\frac{\bar{z}}{e} N_{\underline{\underline{a}}} \neq 0$. Entấo $\varphi$ também é de segunda classe. 
Para $(D-r)$ Impar obtivemos um conjunto de vínculos formado por um número ímpar de vínculos de segunda classe. Con isto, a matriz formada pelos parênteses de Poisson dos vínculos de segunda classe näo ênversivel. Desta maneira, deve existir uma combinaçăto dos vínculos que será de primeira classe.

Seja o conjunto dos vítculos de segunda lasse

$$
\Gamma_{(\xi)}=\left(\varphi, \chi, \Phi_{\underline{H}}\right)=\left(\Gamma_{(A)}, \Gamma_{(B)}, \Gamma_{(\underline{B})}\right) .
$$

Considerando a representação matricial,

$$
\gamma_{(\xi)(\eta)}=\left\{\Gamma_{(\xi)}, \Gamma_{(\eta)}\right\},
$$

sabemos que det $\| \gamma(\xi)$ (n) $\|=0$, já que a ordem de $\gamma$ é impar, mas por ser uma matriz antissimétrica, seu posto será uma unidade inferior à sua ordem, conforme discutimos no Apễndice $\mathrm{A}$. Sendo assim, existe um único autonetor $W^{(\xi)}$ com auto-valor nulo tal que

$$
W^{(k)} \gamma_{(b)(n)}=0
$$

onde

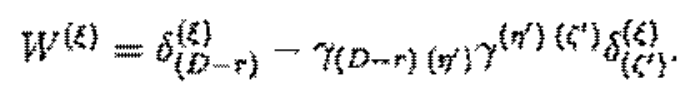

Consideremos a seguinte combinaçăo

$$
\Xi=W^{(t)} \Gamma_{(\xi)}
$$

Então,

$$
\begin{aligned}
\left\{\equiv, \Gamma_{(\xi)}\right\} & =W^{(n)}\left\{\Gamma_{(\eta)} \Gamma_{(\xi)}\right\}+\left\{W^{(n)}, \Gamma_{(\xi)}\right\} \Gamma_{(\eta)} \\
& =W^{(n)} \gamma_{(\eta)}(\xi)=0 .
\end{aligned}
$$

Logo, 


$$
E=\Phi_{\underline{D-r}}-\left\{\Phi_{D-r}, \Gamma_{\left(n^{t}\right)}\right\} \gamma^{\left(n^{\prime}\right)\left(\xi^{\prime}\right)} \Gamma_{\left(\xi^{t}\right)}
$$

é a combinaçăo dos vínculos que é de primeira classe.

Temos $\Gamma_{\left(\xi^{\prime}\right)}=\left(\varphi, X, \Phi_{\underline{u}^{\prime}}\right)$ como o conjunto com vínculos de segunda classe e os vinculos de primeira classe $\Xi$ e $\Pi_{e}$. Assim existem $D+r-5$ graus de liberdade no espaço de fase. 


\section{Capítulo 4}

\section{A Corda Bosônica}

\section{Introdução}

Quando consideramos a partícula relativistica movendo-se no espaco-tempo esta realíza uma trajetória que define uma linha de evolução. No caso de uma corda movendonse no espaço-tempo existe uma superficie de evoluçẫo associtda ao trajeto da corda. Neste caso, a ação do modelo de uma corda será proporcional à área da superficie de evolução. Esta superficie, por estar imersa no espaçótenpo, possui uma métrica induzida pela imersão. A ação, por ser proporcional ama trea esta atea estar relacionada ao determinante da métrica induzida, define a formulação do modelo de corda através da açăo de NambuGoto [26]. Numa outra fomulação, que é equivalente a de Nambu-Goto, temos a açắo de Polyakov, que por ser de primeira orden nas derivadas dos campos, é muito útil no desenvolvimento da Teoria de Cordas em vários aspectos.

Neste trabalho, um aspecto importante é a definiçăo da açăo com o campo do fundo

gravitacional, sendo este campo representado pela tnetrica do espaço-tempo no qual a superficie de evolugăa esta imersa. Neste caso, a açăo e as squaçöes de movimento não envolyem o inverso da métrica do espaç̧o-tempo sendo possivel considerarmos o que ocorre quando a métrica passa a ser degenerada, conforme veremos ao longo das segoes seguintes. 
Vamos construir o formalismo Hamiltoniano e considexar una contagen efetiva dos graus de liberdade para a corda imersa num espacontempo que admite métricas degeneradas. Em analogia aos modelos das partículas, os vinculos presentes irâo depender de algumas propredades dos vetores de base que constituem a métrica degenerada. Entretanto, por ser um objeto estendido comparada a uma particula, surgem propriedades no formalismo Hamiltoniano que tomam o modelo bastante diferente do caso em que a métrica do espaço-tempo nẫo é degenerada.

Apresentaremos na Seção 4.1 o caso em que a corda está imersa num campo de fundo gravitacional arbitrário. Obtemos soluções clássicas que säo independentes da métrica do espaço-tempo quando as invariâncias da af̧̃äo näo săo totalmente fixas. Ao fixarmos as inyariâncias restantes notamos que a métrica deve satisfazer algumas condiçôes, resultando em campos de fundo gravitacionais especificos, sendo que alguns destes podern ser degenerados.

$\mathrm{Na}$ Seçäo 4.2 apresentamos o desenvolvimento do formalismo Hamiltoniano no caso em que a corda está imersa num carnpo de fundo grayitacional nāo degenerado e degenerado, tratados separadamente. No caso degenerado, obtemos vínculos com tuna estrutura algébrica nậo trivial comparada ao caso nãa degenerado. A evoluçâo destes vínculos irá gerar detalhes importantes na forma como a corda interage con a estrutura geométrica do espaço-tempo que admite métricas degeneradas.

\subsection{Algumas Soluções Clássicas para a Corda em Campos de Fundo Gravitacionais}

A açāo de uma cotda ern um fundo gravitacional $G_{z}(X)$, definido en um espaço-tempo de dimensão $D$ onde $(\mu, \nu, 0, \ldots, D-1)$, é dada por

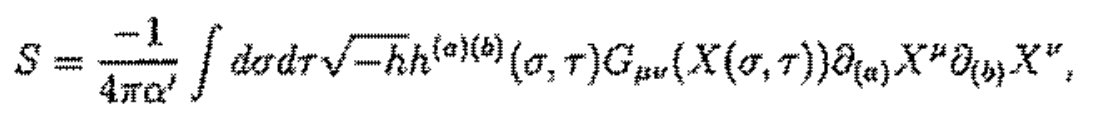


onde $h_{(a)(b)}((a)=0,1)$ é a métrica da superficie de evoluçăo da corda. As equaçöes de movimento para $X^{4}$ e $t^{\text {ab }}$ săo respectivamente

$$
\begin{aligned}
& \frac{1}{\sqrt{-h}} \partial_{(a)}\left(\sqrt{-h} h^{(a)\langle(b)} \partial_{\{b)} X^{\mu}\right) G_{\lambda \mu}+\Gamma_{\mu v \lambda} h^{(a)(b)} \partial_{\{a\}} X^{\mu} \partial_{(b)} X^{\nu}=0 \\
& T_{(a)(b)}=G_{\mu \nu}\left(\partial_{(a)} X^{\mu} \partial_{(b)} X^{\nu}-\frac{1}{2} h_{(a)(b)} h^{\left(a^{\prime}\right)\left(b^{\prime}\right)} \partial_{\left(a^{\prime}\right)} X^{\mu} \partial_{\left(b^{\prime}\right)} X^{\nu}\right)=0 .
\end{aligned}
$$

Observemos que as expressoōes de (4.1) a (4.3) não dependem do inverso de $G_{\mu \nu}$, portanto, estão bem definidas mesmo quando $G_{\mu \nu}$ for degenerada.

No gatge ortonormal, i.e. $h_{a t}=\eta_{a b},(4.2)$ e (4.3) se reduzern a

$$
\begin{aligned}
& G_{\lambda \psi} \hat{O}_{+} \hat{O}_{-} X^{\mu H}+\Gamma_{\mu \nu \lambda} \hat{\partial}_{+} X^{\mu} \hat{\partial} X^{\mu x}=0_{1}
\end{aligned}
$$

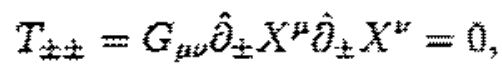

onde $\partial_{t}=\left(\partial_{r} \pm \partial_{0}\right), \partial_{r}=\partial_{0}$ e $\partial_{\sigma}=\partial_{1}$.

Como podemos yer, existem soluçōes para as equaçōes de movimento (4.4) e (4.5) da forma $\hat{\partial}_{4} X^{\beta}=0$ ou $\hat{\partial}_{-} X^{\mu}=0$ que são independentes da métrica $G_{\mu \omega}$ [16]. No ešpaso-tempo de Minkowski em duas dimensöes, campos que satisfazem soluçôes da forma $\tilde{\partial}_{x} X^{\mu}=0$, sẫo chamados de bósons quirais. Nesta terminologia, denotaremos a coordenada da corda envolvida em tais soluçōes como sendo quiral.

Porém, sabemos que a escolha do gauge conforme, relacionado à invariancia de reparametrizaçầ e de Weyl, ainda deixa uma invariancia residual [27] que pode ser fixada corn a escolha de gauge $X^{+}==\frac{1}{\sqrt{2}}\left(X^{o}+X^{D-1}\right)=\left(X_{0}^{+}+P_{T}\right)$, sendo $P$ uma constante. Neste gutuge a coordenada $X^{+}$não é mais quiral, ou seja, $\hat{\partial}_{4} X^{+} \neq 0$. Desta maneira, as soluçöes $\hat{\partial}_{4} X^{i}=0=\hat{\partial}_{+} X^{-}$nas equaçōes (4.4) e (4.5) já näo säo mais exatał, visto que existem termos contendo $\partial_{4} X^{+}$que não irão se anular. Com isto se quisemos obter 
a solução quiral $\hat{\partial}_{+} X^{\mu}=0$ (para $\mu=-, i$ ) devemos impor que o conjunto dos termos contendo $\hat{\partial}_{+} X^{+}$se anule.

As componentes das equaçōes (4.4) contendo os termos $\hat{\partial}_{+} X^{+}=P$ e $\hat{\partial}_{+} X^{\mu}=0$ para $\mu=-, i$ podem ser escritas como

$$
\begin{array}{r}
P\left(2 \partial_{+} G_{+i}-\partial_{i} G_{++}\right)+\left(\partial_{+} G_{-i}+\partial_{-} G_{i+}-\partial_{i} G_{+-}\right) \hat{\partial}_{-} X^{-}+ \\
+\left(\partial_{+} G_{i j}+\partial_{i j} G_{+i]}\right) \hat{\partial}_{-} X^{j}=0 \\
\partial_{+} G_{++}+\partial_{-} G_{++} \hat{\partial}_{-} X^{-}+\partial_{j} G_{++} \hat{\partial}_{-} X^{j}=0 \\
P\left(2 \partial_{+} G_{+-}-\partial_{-} G_{++}\right)+\partial_{+} G_{--} \hat{\partial}_{-} X^{-}+ \\
+\left(\partial_{+} G_{j-}+\partial_{j} G_{-+}-\partial_{-} G_{+j}\right) \hat{\partial}_{-} X^{j}=0 .
\end{array}
$$

Analogamente para (4.5), resulta em

$$
\begin{array}{r}
G_{++}(P)^{2}=0 \\
2 G_{+-} P \hat{\partial}_{-} X^{-}+2 G_{+i} P \hat{\partial}_{-} X^{i}+ \\
+2 G_{-i} \hat{\partial}_{-} X^{-} \hat{\partial}_{-} X^{i}+G_{--} \hat{\partial}_{-} X^{-} \hat{\partial}_{-} X^{-}+G_{i j} \hat{\partial}_{-} X^{i} \hat{\partial}_{-} X^{j}=0
\end{array}
$$

As equaçōes de (4.6) à (4.8) possuem soluções que irão depender da forma de $G_{\mu \nu}$. Com isto, uma solução destas equações (independente de $X^{+}$) é dada por:

$$
\begin{array}{lll}
G_{--}=\tilde{g}_{--} & G_{-i}=\bar{g}_{-i} & G_{+j}=\bar{g}_{i j} \\
G_{+i}=\partial_{i} \bar{h} & G_{+-}=\partial_{-} \bar{h} & G_{++}=0
\end{array}
$$

onde $\tilde{g}$ e $\tilde{h}$ são funçōes apenas de $X^{-}$e $X^{i}$. 
Temos, entäo, uma forma para $G_{z \neq \nu}$ que permite a soluçào quiral para as componentes $X^{-}$e $X^{i}{ }_{3}$ com todas as invariâncias da açăo (4.1) completamente fixas.

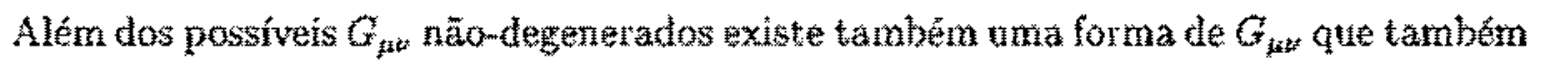
satisfaz (4.6) à (4.8), porém com determinante nulo. As componentes de $G_{\mu v}$ são

$$
\begin{array}{lll}
G_{+-}=a h & G_{++}=0 & G_{-i}=\left[a_{i} X^{+}+g_{i}\left(X^{i}\right)\right] G_{+-} \\
G_{+i}=a_{i} h+b_{i} h & G_{+--}=0 & G_{i j}=\frac{G_{+(i i} G_{-j)}}{G_{+-}}
\end{array}
$$

onde $a_{i}$ são constantes eh $h=h\left(X^{-}, X^{i}\right)$. Vemos, então, a presença direta de uma métrica degenerada na soluçăo das equaçôe de movimento da corda em campo de fundo gravitacional, desce que as coordenadas da corda sejam do tipo quiral.

Consideremos a seguir o formalismo Hamiltoniano em que será considerada a presenç̧a de campos de fundo com métricas nâo degeneradas e degeneradas.

\subsection{Formalismo Hamiltoniano}

\subsubsection{Campo de Fundo Gravitacional não Degenerado}

Para a conştruçấo da Hamiltoniana associada a açăo (4.1) consideremos as densidades de momento

$$
\begin{aligned}
& P_{(a)(b)}=\frac{\partial L}{\partial h^{(a)(b)}}=0
\end{aligned}
$$

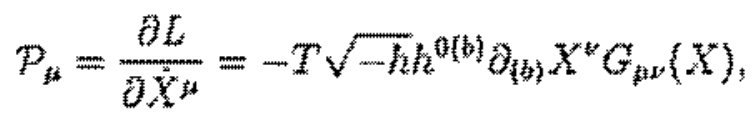

onde $T=\frac{1}{2 \pi \omega^{2}}$ 
No caso em que det $G_{y z} \neq 0$, temos o seguinte conjunto de vinculos primários:

$$
\Phi_{(a)(b)}=p_{(a)(b)}=0
$$

Vamos introduzir os seguintes parểnteses de Poisson

$$
\begin{aligned}
& \left\{h^{(a)(b)}(\sigma), p_{(c)(d)}\left(\sigma^{*}\right)\right\}_{\gamma}=\frac{1}{2}\left(\delta_{(s)}^{(a)} \delta_{(d)}^{(b)}+\delta_{(c)}^{(b)} \delta_{(d)}^{(a)}\right) \delta\left(\sigma-\sigma^{\prime}\right) \\
& \left\{X^{\mu}(\sigma), P_{z}\left(\sigma^{\prime}\right)\right\}_{\tau}=\delta_{k}^{\mu} \delta\left(\alpha-\sigma^{\prime}\right)
\end{aligned}
$$

Vamos calcular a dependềncia temporal dos vínculos em (4.14) atravếs da Hamiltoniana primária $H$

$$
H=\int_{0}^{2 \pi} d \sigma\left(\mathcal{H}_{c}+\lambda^{(a)(b)} \Phi_{(a)(b)}\right),
$$

onde $\lambda^{(a)(b)}$ são multiplicadores de Lagrange $\mathrm{e}$

$$
\mathcal{H}_{c}=\frac{-1}{2 h^{00}}\left[\frac{(-h)^{-1 / 2}}{T} \mathcal{p}_{\mu} \mathcal{p}_{\nu} G^{\mu \nu}+2 h^{01} \mathcal{P}_{\mu} X^{\prime \mu}+T(-h)^{-1 / 2} X^{\prime \mu} X^{\prime \nu} G_{\mu \nu,}\right]
$$

onde $X^{*}=\frac{\partial}{\partial \sigma} X^{\mu}$. Da evolurâa temporal de $\Phi_{a b}$, cujos detalhes dos cálculos se encontram no apèndice $B$, obtemos os seguintes vínculos secundários:

$$
\begin{aligned}
& \varphi_{1}=\frac{l}{T} P_{\mu} x^{\mu}=0 \\
& \varphi_{z}=\frac{1}{2}\left(\frac{1}{T^{2}} p_{\mu} p_{\nu} G^{\mu t+}+X^{*} X^{\prime}{ }^{\prime} G_{\mu \nu}\right)=0
\end{aligned}
$$

A evolução temporal de $(4.18)$ e (4.19) näo geva novos vínculos e todos os vínculos da teoria säo de primeira classe, como podemos constatar atravếs da álgebra 


$$
\begin{aligned}
& \left\{\varphi_{1}(\sigma), \varphi_{1}\left(\sigma^{\prime}\right)\right\}=T^{-i}\left[\varphi_{1}(\sigma)+\varphi_{1}\left(\sigma^{\prime}\right)\right] \partial_{\sigma} \delta\left(\sigma-\sigma^{\prime}\right) \\
& \left\{\varphi_{1}(\sigma), \varphi_{2}\left(\sigma^{\prime}\right)\right\}=T^{-1}\left[\varphi_{2}(\sigma)+\varphi_{2}\left(\sigma^{\prime}\right)\right] \partial_{\sigma} \delta\left(\sigma-\sigma^{\prime}\right) \\
& \left\{\varphi_{2}(\sigma), \varphi_{2}\left(\sigma^{\prime}\right)\right\}=T^{-1}\left[\varphi_{1}(\sigma)+\varphi_{1}\left(\sigma^{\prime}\right)\right] \partial_{\sigma} \delta\left(\sigma-\sigma^{n}\right)
\end{aligned}
$$

\subsubsection{Campo de Fundo Gravitacional Degenerado}

Consideremos uma métrica degenerada, num espaçotempo de $D$ dimensōes, dada por

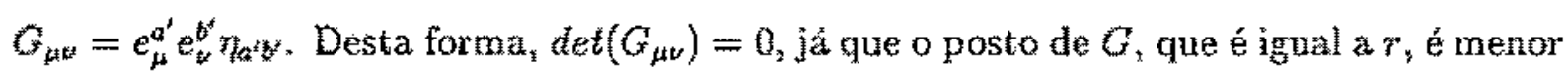
que $D$.

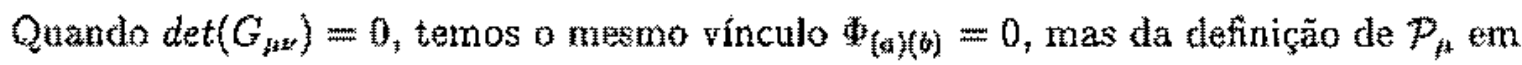
(4.13), vemos que para eliminarmos $\dot{X}^{\nu}$ necessitamos da inversa de $G_{\mu \nu}$. Como esta nāo existe, iremas mostrar que surge um nowo vinculo.

Vamos escrever $P_{\mu}$ como:

$$
\begin{aligned}
& P_{\mu}=\Delta_{a^{z}} e_{\xi k}^{a^{z}},
\end{aligned}
$$

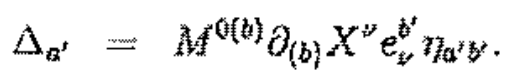

Considerando uma divisão do indice $\mu$ da forma $\mu=\left(\mu^{t}, \underline{\mu}\right)$, onde $\mu^{*}=0,1, \ldots, t-1 ; \underline{\mu}=$ $r, \ldots, D-1$, temos

$$
\begin{aligned}
& P_{\mu^{\prime}}=\Delta_{a^{t}} e_{\mu^{\prime}}^{a^{z}} \\
& p_{\underline{\mu}}=\Delta_{a^{2}} e_{\underline{\mu}}^{a^{x}} .
\end{aligned}
$$


Porém, no setor $\left(a^{*} ; \mu^{t}\right)$ podemos definir uma inversa dada por êt, entăo

$$
\begin{aligned}
& e_{\mu^{\prime}}^{a^{\prime}} e_{b^{\prime}}^{u^{\prime}}=\delta_{b^{\prime}}^{a^{\prime}} \\
& e_{\mu^{\prime}}^{a^{\prime}} \hat{e}_{\mathrm{a}^{\prime}}^{y^{\prime}}=\delta_{v^{\prime}}^{y^{z}}
\end{aligned}
$$

De (4.24) temos que

$$
\Delta_{n^{3}}=\vec{e}_{a^{i}}^{u^{*}} p_{f^{3}}
$$

Logo

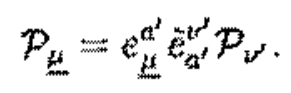

Temos, entäo, o seguinte vinculo primário:

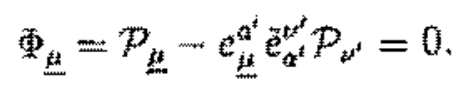

Devemos notar ainda que

$$
\begin{aligned}
& \Phi_{\underline{\underline{\mu}}}=u_{\underline{\underline{B}}}^{\lambda} \mathcal{P}_{\lambda},
\end{aligned}
$$

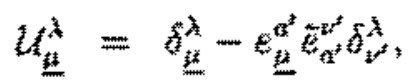

onde (4.32) corresponde a uma representaçäo dos auto-vetores nulos de $G_{\mu v}$, ou seja,

$$
U_{\mu}^{\lambda} G_{\lambda \nu}=0
$$

Sendo assim, $G_{\lambda y}$ possui $D-r$ auto-vetores com auto-valor zero, i.e., $D-r$ auto-vetores nulos.

Para a mếtrica degenerada em estudo, obtemos a densidade de hamiltoniana canônica $\mathcal{H}_{c}$, cujos cálculos estão efetuados no apêndice $\mathrm{B}$,

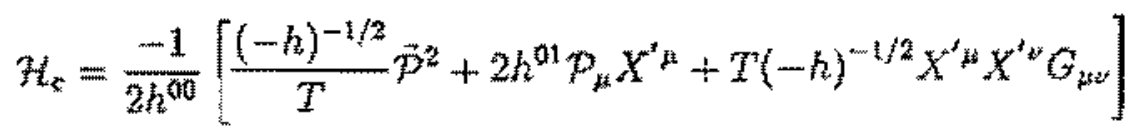


onde,

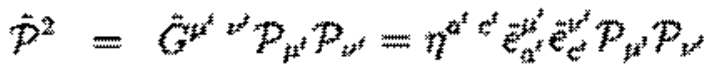

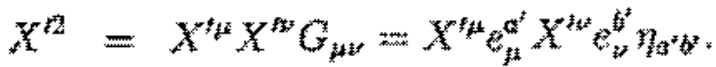

Como já obtivemos todos os vínculos primârios, vamos evoluir estes vínculos com a Hamiltoniana primária $H_{p}$

$$
H_{p}=\int\left(\mathcal{H}_{c}+\lambda^{[a](b)} \mathcal{P}_{(a)(b)}+\lambda^{\underline{u}} \Phi_{k}\right) d \sigma^{*}
$$

Inicialmente vamos evoluir o vínculo $p_{(a)(b)}=0$. Porén devemos antes notar que

$$
\left\{\mathcal{P}_{(a)(b)}, \Phi_{\nu}\right\}=0 .
$$

Como $\mathcal{H}_{c}$ apresenta a mesma forma do caso näo degenerado, a partir de

$$
\dot{P}_{\{a\}(b)}=0
$$

obtemos os seguintes vínculos secundários

$$
\begin{gathered}
\varphi_{1}=\frac{1}{T} p_{\mu} X^{\prime \mu}=0 \\
\varphi_{2}=\frac{1}{2}\left(\frac{\tilde{p}^{2}}{T^{2}}+X^{\prime 2}\right)=0
\end{gathered}
$$

conforme demonstrado no apểndice $B$.

A partir destes últimos vínculos podemos reescrever $H_{c}$ como

$$
H_{c}=-\frac{\Upsilon}{h^{00}}\left[(-h)^{-1 / \alpha_{p}} \varphi_{2}+h^{0} p_{1}\right] .
$$


Evoluindo 㖉,

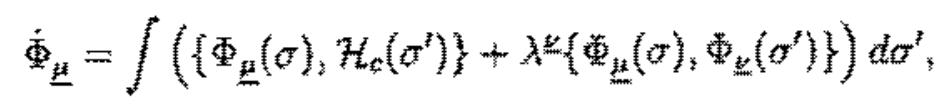

temos

$$
\left\{\Phi_{\underline{a}}(\sigma), \mathcal{H}_{c}\left(\sigma^{\prime}\right)\right\}=c_{1}\left\{\Phi_{\underline{a}}(\sigma), \varphi_{1}\left(\sigma^{5}\right)\right\}+c_{2}\left\{\Phi_{\underline{a}}(\sigma), \phi_{2}\left(\sigma^{\prime}\right)\right\}
$$

onde

$$
\begin{gathered}
c_{1}=-T \frac{h^{01}}{h^{00}}, \\
c_{2}=-\frac{T(-h)^{-1 / 2}}{h^{00}} .
\end{gathered}
$$

Os parênteses de Poisson restantes, cujos cálculos se encontram no Apêndice C, sẵo dados por:

$$
\begin{aligned}
& \left\{\Phi_{\underline{\mu}}(\sigma), \varphi_{1}\left(\sigma^{\prime}\right)\right\}=-\Phi_{\underline{\mu}}\left(\sigma^{\prime}\right) \partial_{r^{\prime}} \sigma\left(\sigma-\sigma^{\prime}\right)
\end{aligned}
$$

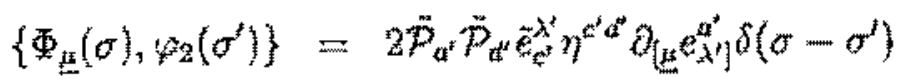

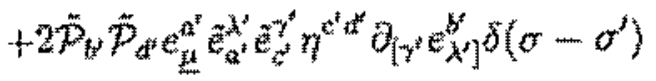

$$
\begin{aligned}
& -v_{ \pm}^{a}(\sigma) \partial_{1 p} G_{\lambda p}(\sigma) X^{\prime \lambda} X^{\prime} p(\sigma) \delta\left(\sigma-\sigma^{\prime}\right) \\
& +2 U_{\mu}^{\mu}(\sigma) G_{\mu D}\left(\sigma^{\prime}\right) X^{x}\left(\sigma^{\prime}\right) \partial_{\sigma} \delta\left(\sigma-\sigma^{t}\right) \\
& \left\{\Phi_{\underline{y}}(\sigma), \Phi_{z}\left(\sigma^{n}\right)\right\}=M_{\underline{z}}(\sigma) \delta\left(\sigma-\sigma^{n}\right)
\end{aligned}
$$

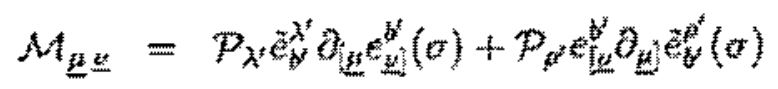




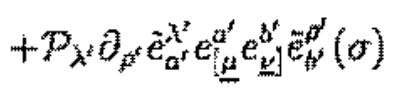

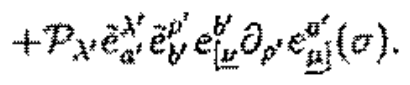

Para o último termo de $(4,47)$ vamos utilizar que

$$
\begin{aligned}
& \partial_{\sigma}\left(U_{\underline{u}}^{\mu}\left(\sigma^{\prime}\right) G_{\lambda \mu}\left(\sigma^{\prime}\right) X^{\prime}{ }^{\lambda}\left(\sigma^{\prime}\right) \delta\left(\sigma-\sigma^{\prime}\right)\right)=0 \\
& =\partial_{\sigma}\left(U \mu(\sigma) G_{\lambda \mu}\left(\sigma^{\prime}\right) X^{*}\left(\sigma^{\prime}\right) \delta\left(\sigma-\sigma^{\prime}\right)\right) \\
& =\partial_{\sigma} U_{\underline{x}}^{\mu}(\sigma) G_{\lambda \mu}\left(\sigma^{\prime}\right) X^{\prime \lambda}\left(\sigma^{\prime}\right) \delta\left(\sigma-\sigma^{\prime}\right) \\
& +U_{\underline{\mu}}^{\mu}(\sigma) G_{\lambda_{p}}\left(\sigma^{\prime}\right) X^{2 \lambda}\left(\sigma^{\prime}\right) \partial_{\sigma} \delta\left(\sigma-\sigma^{\prime}\right) \text {. }
\end{aligned}
$$

Então,

$$
V_{\underline{\underline{k}}}^{\mu}(\sigma) G_{\lambda \lambda^{2}}\left(\sigma^{\prime}\right) X^{\prime \lambda}\left(\sigma^{\prime}\right) \partial_{\alpha} \delta\left(\sigma-\sigma^{\prime}\right)=-\partial_{\sigma} U_{\underline{\nu}}^{\mu}(\sigma) G_{\lambda \mu}\left(\sigma^{\prime}\right) X^{\prime \lambda}\left(\sigma^{\prime}\right) \delta\left(\sigma-\sigma^{\prime}\right)
$$

Com isto, podemos reescrever (4.47) como

$$
\begin{aligned}
& \left\{\omega_{\mu_{\alpha}}(\sigma), \varphi_{2}\left(\sigma^{t}\right)\right\}=n_{\underline{\mu}}(\sigma) \partial\left(\sigma-\sigma^{t}\right),
\end{aligned}
$$

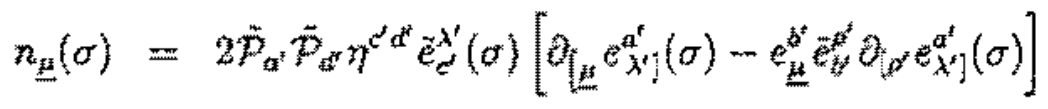

$$
\begin{aligned}
& -V_{\underline{\mu}}^{\beta} \partial_{\beta} G_{\lambda \rho} X^{* \lambda} X^{*} \rho(\sigma)-2 \partial_{s} U_{\underline{\mu}}^{*} G_{\lambda \nu} X^{\prime \lambda}(\sigma) \text {. }
\end{aligned}
$$

Destic forma, segue que

$$
\begin{aligned}
& \left\{\Phi_{3}(\sigma), \mathcal{H}_{c}\left(\sigma^{\prime}\right)\right\}=c_{1}\left(-\Phi_{\underline{4}}\left(\sigma^{\prime}\right) \partial_{r} \delta\left(\sigma \cdots \sigma^{\prime}\right)\right)+c_{2} \tilde{t}_{\mu}\left(\sigma^{\prime}\right) \delta\left(\sigma-\sigma^{\prime}\right) \\
& =N_{\underline{s}}\left(\sigma^{3}\right) \delta\left(\sigma-\sigma^{t}\right) \\
& N_{\mathrm{a}}(\sigma)=c_{2}(\sigma) n_{\underline{\mu}}(\sigma)
\end{aligned}
$$


Considerando todas as contribuiçâes de $(4.42)$, ficamos com:

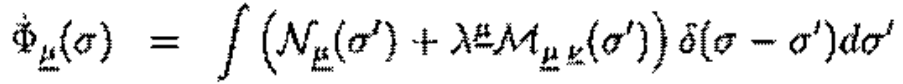

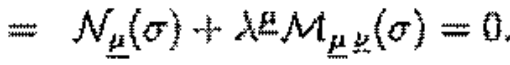

Observamos que (4.55) possui uma forma correspondente aos casos das partículas relativisticas e não relativísticas. Sendo assim, analisaremos os casos etn que a dinénsão de $\mathcal{M}$ é par ou ímpar.

\subsubsection{Caso $D-r$ Par}

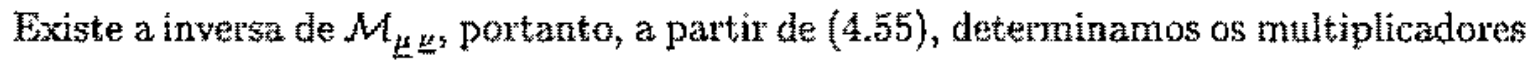
de Lagrange $\lambda^{\sharp}=-\mathcal{M} \underline{\underline{H}} \mathcal{N}_{\underline{z}}$.

Vejamos como ficam as evoluçōes dos vínculos secundários $\varphi_{1}$ e $\varphi_{2}$.

$$
\begin{gathered}
\dot{\varphi}_{1}(\sigma)=\int\left(\left\{\phi_{1}(\sigma), \mathcal{H}_{c}\left(\sigma^{\prime}\right)\right\}+\lambda^{*}\left\{\varphi_{1}(\sigma), \phi_{2}\left(\sigma^{\prime}\right)\right\}\right) d \sigma^{\prime} \\
\dot{\varphi}_{2}(\sigma)=\int\left(\left\{\varphi_{2}(\sigma), \mathcal{H}_{c}\left(\sigma^{\prime}\right)\right\}+\lambda^{3}\left\{\varphi_{2}(\sigma), \Phi_{2}\left(\sigma^{\prime}\right)\right\}\right) d \sigma^{\prime} \\
\left\{\varphi_{1}(\sigma), \mathcal{H}_{c}\left(\sigma^{\prime}\right)\right\}=c_{1}\left\{\varphi_{1}(\sigma), \varphi_{1}\left(\sigma^{\prime}\right)\right\}+c_{2}\left\{\varphi_{1}(\sigma), \varphi_{2}\left(\sigma^{\prime}\right)\right\} \\
\left\{\varphi_{2}(\sigma), \mathcal{H}_{c}\left(\sigma^{\prime}\right)\right\}=c_{1}\left\{\varphi_{2}(\sigma), \varphi_{1}\left(\sigma^{\prime}\right)\right\}+c_{2}\left\{\varphi_{2}(\sigma), \varphi_{2}\left(\sigma^{\prime}\right)\right\}
\end{gathered}
$$

No apêndice $C$ apresentamos os cálculos envolvidos para a obtençäo de seguinte ágebra dos vinculos:

$$
\left\{\varphi_{1}(\sigma), \varphi_{1}\left(\sigma^{\prime}\right)\right\}=\left[\phi_{1}(\sigma)+\varphi_{1}\left(\sigma^{\prime}\right)\right] \partial_{\sigma} \delta\left(\sigma-\sigma^{\prime}\right)
$$




$$
\begin{aligned}
& \left\{\varphi_{1}(\sigma), \varphi_{2}\left(\sigma^{\prime}\right)\right]=\left[\varphi_{2}(\sigma)+\varphi_{2}\left(\sigma^{\prime}\right)\right] \partial_{\sigma} \delta\left(\sigma-\sigma^{\prime}\right) \\
& \left\{\varphi_{2}(\sigma), \varphi_{2}\left(\sigma^{\prime}\right)\right\}=\left[\varphi_{1}(\sigma)+\varphi_{1}\left(\sigma^{\prime}\right)\right] \partial_{\sigma} \delta\left(\sigma-\sigma^{\prime}\right)
\end{aligned}
$$

Então, podemos concluir que

$$
\dot{\varphi} \dot{\varphi}_{1}=0
$$

näo gera novos vínculos.

Porém, de

$$
\left\{\varphi_{2}(\sigma), \Phi_{L}\left(\sigma^{\prime}\right)\right\}=-n_{\underline{L}}\left(\sigma^{\prime}\right) \delta\left(\sigma-\sigma^{*}\right)=-\frac{\mathcal{N}_{\underline{L}}}{c_{2}}\left(\sigma^{\prime}\right) \delta\left(\sigma-\sigma^{\prime}\right)
$$

segue que

$$
\begin{aligned}
\dot{\varphi}_{z} & =-\int \lambda \frac{\mathcal{N}_{\underline{\underline{z}}}}{c_{2}}\left(\sigma^{\prime}\right) \delta\left(\sigma-\sigma^{\prime}\right) d \sigma^{t} \\
& =-\frac{1}{c_{2}} \lambda^{\underline{\nu}} \mathcal{N}_{u}(\sigma) .
\end{aligned}
$$

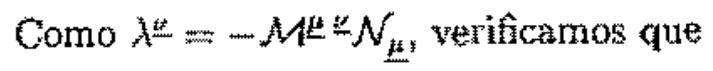

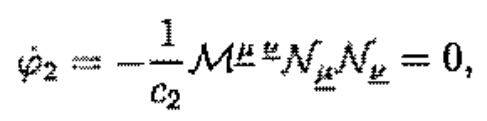

näo gera nowos vínculos.

Temos então um conjunto com um número impar de vínculos de segunda clässe, $\Phi_{\mu}$ e $p_{2}$. Desta forma, varros considerar uma combinação dos vinculos que seja de primeira classe

$$
\phi=\xi_{\varphi_{2}}+f \underline{\mu} \underline{\Phi}_{\underline{\mu}}
$$


e determinar os coeficientes $\xi$ e $f^{\mu}$ a partir de

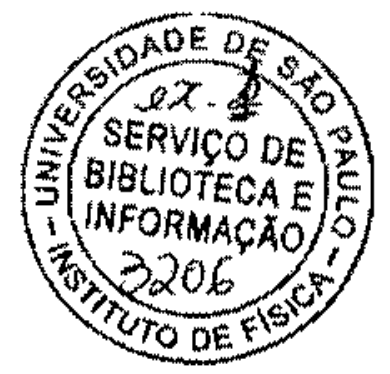

$$
\begin{aligned}
& \left\{\phi, \varphi_{2}\right\}=f \underline{\mu}\left\{\Phi_{\underline{\underline{\mu}}}, \varphi_{2}\right\}=f^{\underline{\mu}} \mathcal{N}_{\underline{\mu}} \delta=0 \\
& \left\{\phi, \Phi_{\underline{\underline{e}}}\right\}=\xi\left\{\varphi_{2}, \Phi_{\underline{\underline{p}}}\right\}+f_{\underline{\mu}} \mathcal{M}_{\underline{\underline{\mu}} \underline{\underline{p}}}^{\delta}=0 .
\end{aligned}
$$

Temos que a soluçâo para (4.68) é dada por

$$
f \underline{H}=\mathcal{N}_{\underline{\alpha}} M{ }^{2} \underline{\underline{E}} \quad \xi=c_{2}
$$

Entâo,

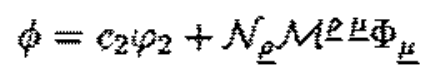

E. combinaçăo procurada.

Ficamos com um número par de vínculos de segunda classe definidos por in $_{\text {e }}$ com os vínculos de primeixa classe dados por $\phi, \varphi_{1}$ e $\mathcal{P}_{(a)(b)}$. Então existem $D+r-4$ graus de liberdaxie no espaço de fase.

\subsubsection{Caso $D-r$ Ímpar}

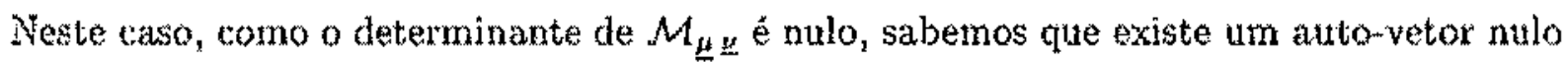

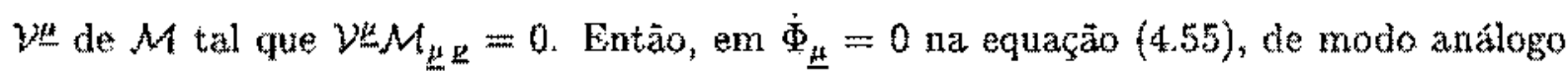
ao que foi realizado na partícula relativística, surgirá o vínculo

$$
x=V \underline{\underline{\mu}} N_{\underline{\underline{\mu}}}=0
$$

onde a utilizarmos a representaça de $\not{ }^{2}$, obtida no apendice $A$, obtemos

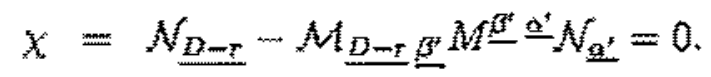


Utilizando novamente a analogia da partícula relativísica, porém desta vez comparando com a equação (3.39) do Capítulo 3 , o valor de $\lambda \Perp$ dado por

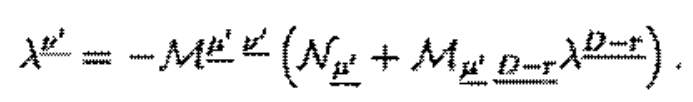

Vamos, agora, determinar a evoluçăo temporal de $\chi, \varphi_{1}$ e $\psi_{2}$.

Analogamente ao que foi realizado no caso $D-r$ par, a evolução temporal de $\hat{\varphi}_{1}$ näo gera novos vínculos. Para $\varphi_{2}$, temos

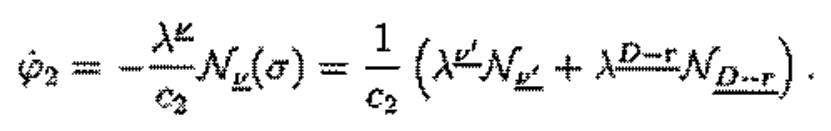

Substituindo $\lambda^{u^{2}}$ temos

$$
\dot{\varphi}_{2}=\lambda^{D-r} \chi=0
$$

ou seja, nenhum vínculo é gerado.

Vejamos a evolução de $\chi$

$$
\begin{gathered}
\dot{x}=\left\{\chi, H_{p}\right\}=\int\left(\left\{\chi(\sigma), H_{c}\left(\sigma^{\prime}\right)\right\}+\lambda^{\prime \prime}\left\{\chi(\sigma), \varphi_{2}\left(\sigma^{\prime}\right)\right\}\right) d \sigma^{3}, \\
\left\{\chi, H_{c}\left(\sigma^{\prime}\right)\right\}=c_{1}\left\{\chi(\sigma), \varphi_{1}\left(\sigma^{\prime}\right)\right\}+c_{2}\left\{\chi(\sigma), \varphi_{2}\left(\sigma^{\prime}\right)\right\} .
\end{gathered}
$$

Para ficar com uma forma conveniente, análoga à partícula relativística, consideremos

$$
H_{c}\left(\sigma^{n}\right)=\frac{\bar{c}}{2} \varphi
$$

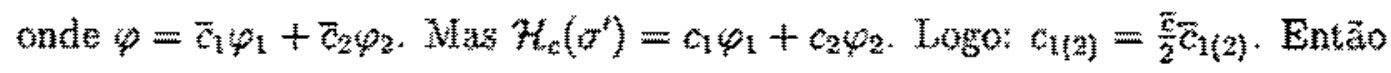

$$
\left\{\chi, \mathcal{H}_{c}\left(\sigma^{\prime}\right)\right\}=\left\{\chi, \frac{\bar{c}}{2} \varphi\right\}
$$

Ainda temos 


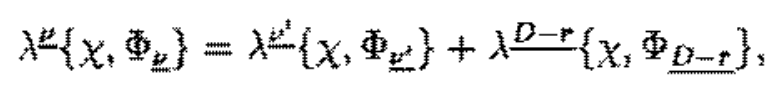

$\log 0$

$$
\dot{\chi}(\sigma)=\int\left(\left\{\chi(\sigma), \frac{\bar{c}}{2} \varphi\left(\sigma^{\prime}\right)\right\}+\lambda^{\mu^{\prime}}\left\{\chi(\sigma), \Phi_{\underline{u}^{\prime}}\left(\sigma^{\prime}\right)\right\}+\lambda \stackrel{D-r}{D-}\left\{\chi(\sigma), \underline{\Phi_{-r}}\left(\sigma^{\prime}\right)\right\}\right) d \sigma^{\prime}
$$

Nesta última expressäo, vamos substituir $\lambda^{2}$, de modo análogo ao que foi realizado na partícula relativística, resultando em;

$$
\begin{aligned}
& \dot{x}(\sigma)=\int\left(\left\{x(\sigma), \frac{\vec{c}}{2} \varphi\left(\sigma^{\prime}\right)\right\}-\mathcal{M}^{E^{t} E^{t} N_{p}}\left\{x, \Phi_{\underline{A}^{t}}\right\}\right.
\end{aligned}
$$

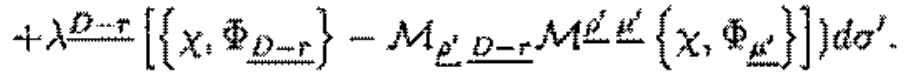

Devemos notar que

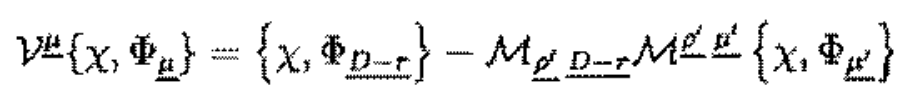

nầo se anula. Entāo

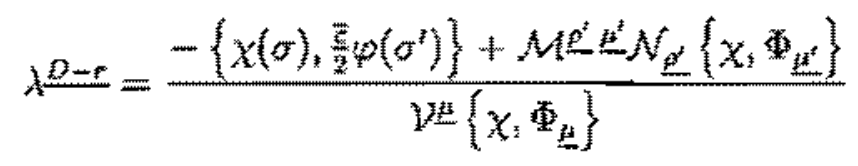

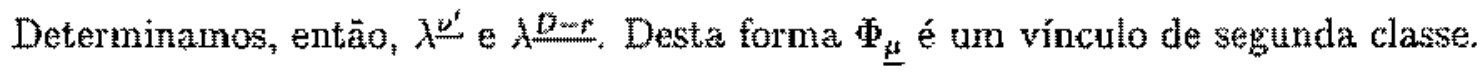

Como a Hamiltoniana estendida apresenta a mesma forma da particula relativistica conclúmos que o multiplicador de Lagrange $\mu$ é nulo, o que permite determinar $\chi$ como vínculo de seguncla classe. Como $\left\{\varphi_{2}(\sigma), \Phi_{\mu}\left(\sigma^{\prime}\right)\right\} \neq 0$ concluínos que $\varphi_{2}$ é de segunda classe.

Resta analisar $\left\{\varphi_{1}, \chi\right\}$. Temos que, 


$$
\begin{aligned}
& \left\{\varphi_{1}, \chi\right\}=\left\{\mathcal{P}_{\mu} X^{-\mu}, V^{\mu} N_{\mu}\right\}
\end{aligned}
$$

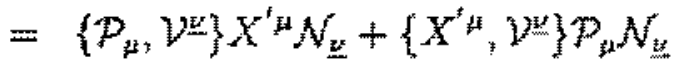

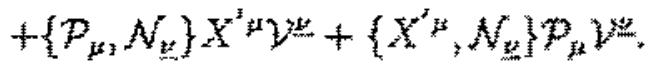

Nâo é possivel determinar se (4.85) anula-se ou nāo pois o călculo envolvito depende explicitamente da mêtrica.

Então, sé $\left\{\varphi_{1}, \chi\right\} \neq 0$, teremos um número par de vínculos de segunda classe. Neste caso existem $D+r-3$ graus de liberdade no espaço de fase.

Por outro lado, se $\left\{p_{1}, \chi\right\}=0$, teremos um número ímpar de vinculos de segunda classe e neste caso existe uma combinação dos vínculos que é de primeira classe. Como a estrutura dos vínculos e evoluçôes deste são similares ao da partícula relativistica, a combinaţão dos vínculos, denominada $\Xi$, será da mesma forma. Assim, temos os vínculos de segunda classe $\Phi_{\underline{u^{\prime}}}, \varphi_{2}$ e $\chi$ e os vínculos de primeira classe $\varphi_{1}, \mathcal{P}_{(a)(b)}$ e $\Xi$. Neste caso existem $D+r-3$ graus de liberdade no espaço de fase.

Podemos concluir que a contagem dos graus de liberdade, que é definida localmente para cada ponto ao longo de $\sigma$, é independente do valor de $\left\{\varphi_{1}(\sigma), \chi\left(\sigma^{\prime}\right)\right\}$.

Adicionalmente, conclúmos que o estudo inicial das particulas em espaços com métricas degeneradas, foi bastante útil para auxiliar o desenwolvimento da anlgebra dos vinculos no caso da corda sendo que esta apresenta uma estrutura de vinculos mais complexa. A estrutura dos vínculos das partículas se assemelhando con a estrutura do caso da corda, serviu de comparaça para também determinar a combinaçäo correta dos vínculos que deverian ser de primeira classe, como também para determinar corretanente as evoluçöes dos vínculos primảitos e secundánios. Estas evoluções por serem constitudas de equaçoes näo triviais, só foram simplificadas através de uma analogia com respeito ao tratamento dos vínculos de um modelo simplificado em relaşâ ao da corda, ou seja, o das partículas em espaços com métricas degeneradas. 
Com o uso da particula näo relativistica em espaços com métricas degeneradas, que possui uma estrutura de vínculos similar ao da corda, conseguimos resolver de forma con-

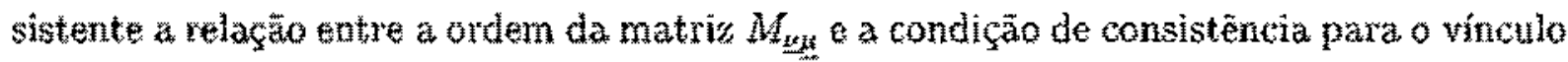
$\Phi_{\mu^{*}}$ Entretanto, por rä́o apresentar analogia aos vínculos secundários da corda, i.e., $\phi_{1}$ e. pq $_{2}$ uma generalizaçäo do modelo para o caso relativístico, trouxe condiçōes e motivações adicionais. No caso relativistico desenvolvido no Capítulo 3, o vínculo secundário para a partícula, ou seja, o vinculo $\varphi$, serve como analogia para simplificar os vínculos secundários da corda. Esta simplifiçą̧ão é útil prinçipalmente com respeito a consistência dos vínculos para o caso em que os vetores de base não são integráveis e quando $M_{\underline{\underline{\mu}} \text { não }}$ possui inversa.

\subsection{Isometrias na Corda com Métrica Degenerada e Vínculos de Primeira Classe}

Conforme vimos nos modelos das partículas descritos nos Capítulos 2 e 3 , a condição

$$
\partial_{p} e_{v}^{a_{2}^{2}}=0
$$

tornava os vinculos de primeira classe. Se considerarmos $\partial_{[\mu} e_{u]}^{a^{t}}=0$ para a corda, obtemos a partir de (4.49), (4.52) e (4.54) que

$$
\begin{aligned}
& M_{\underline{p} \underline{u}}(\sigma)=0 \\
& N_{L}(\sigma)=c_{2}\left(-U_{p}^{a} \partial_{\mu} G_{\lambda p} X^{\alpha} X^{*} \rho(\sigma)\right. \\
& \left.-2 \frac{\partial U_{p}^{\mu}}{\partial \sigma} G_{\lambda \mu} X^{2}(\sigma)\right) \text {. }
\end{aligned}
$$

Esta forma de $\mathcal{N}_{ \pm \underline{4}}$ não permite que todos os vínculos da teoria sejam de primeira classe. Porém, devemos notar que os termos restantes em $N_{\mu}$ podem ser reescritos de una forma especial se considerarmos que a métrica degenerada implica em uma isometria no modelo. 
Vamos considerar a condiçāo de isometria na forma

$$
\mathcal{U}_{\underline{s}}^{\mu} \partial_{\mu} G_{\lambda \rho}+C_{\lambda \mu} \partial_{\rho} \mathcal{U}_{\underline{u}}^{\mu}+G_{\mu \rho} \partial_{\lambda} \mathcal{U}_{\underline{u}}^{\mu}=0
$$

onde $\mathcal{U}_{\mathrm{z}}$ representa um auto-vetor nulo de $G_{\lambda, g}$.

Partindo de (4.88) temos que

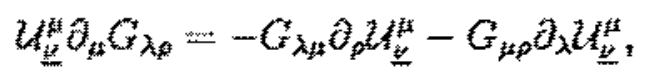

onde podemos utilizar

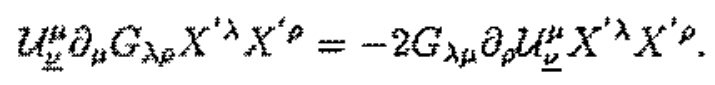

Mas

$$
\partial_{p} \mathcal{U}_{\underline{\underline{p}}}^{\mu} X^{\mathrm{p}} \mathrm{p}=\frac{\partial \mathcal{U}_{\underline{\underline{w}}}^{\mu}}{\partial \sigma}
$$

$\log 0$

$$
\mathcal{U}_{\underline{\mu}}^{\mu} \partial_{\mu} G_{\lambda \rho} X^{\prime \lambda} X^{\prime \rho}=-2 \frac{\partial \mathcal{U}_{\underline{\underline{p}}}^{\mu}}{\partial \sigma} G_{\lambda \mu} X^{\prime \lambda}
$$

Substituindo $(4.92)$ en $(4.87)$, temos $\mathcal{N}_{2}(\sigma)=0$.

Assin temos $\dot{1}_{\underline{u}}=0$, que näo gera novos vínculos e ficamos com um conjunto de

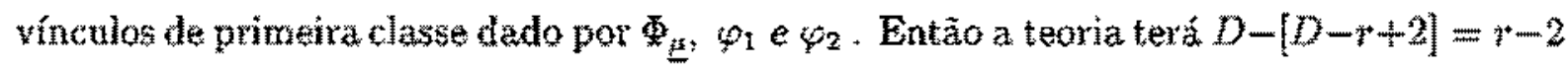
graus de liberdade no espaço de configuração.

Com este resultado podemos concluir que a análise da isometria para a métrica dègenerada no caso da partícula não relativistica foi bastante útil para o caso da corda, servindo como motivacẵo na obtenção dos vínculos de primeira classe. 
Apesar da condiçāo de integrabilidade dos vetores de base permitir una obtenção imediata dos vínculos de primeira classe para as particulas relativisticas e näo relativíticas, o mesmo näo ocorreu para o caso das cordas, visto que a condiçăo de integrabilidade năo tornou todos os vínculos de primeira classe. Neste ponto um recusso importante, que é a isometria associada à métrica degenerada, permitiu a obtençấo completa dos vínculos de primeira classe da corda con métrica degenerada.

Além disto, no caso da corda com métrica degenerada, os vínculos de primeira classe só estão presentes para o caso em que ocorre a isometria em termos do vetores de Killing, sendo estes identificados com os auto-vetores nulos da métrica degenerada.

Adicionalmente, a existeñncia de isometrias no modelo da corda em campo de fundo gravitacional, é de importäncia fundamental para a formulação de açỏes duais $[28,29,30]$. Nesta formulação, a ação obtida a partir de transformaçōes especificas nas componentes da mêtrica inicial, ế classicamente equivalentente à ação inicial, sendo chamada de ação dual. Porém o procedimento utilizado, não utiliza o inverso da métrica. Sendo assim, é de se esperar que uma açäo dual à açăo com métrica degenerada possa dar outras interpretaçöes, neste contexto de dualidade, às métricas degeneradas na teoria de cordas.

\subsection{Invariância Conforme em Nivel Quântico}

Uma vez encontrado um sistema de vínculos de primeira classe na teoria de cordas, podemos verificar se a simetria gerada por estes ê preservada em nível quầntico, e, se năo for, que condiçōes devem ser impostas para que seja.

No caso usual em que a métrica do espaço-tempo possui inversa, a álgebra dos vínculos em nivel quântico é estudada em [31, 32], onde são obtidas condiçōes sobre os campos de fundo de modo que a ágebra continue de primeiza classe, implicando nas condições de invariância conforme em nivel quăntico encontradas em [3:3]. 
Entretanto é possível tratar a âlgebra dos vínculos para a corda com métrica degenerada utilizando método descrito em [32], em que é usado como ponto de partida a relaçăo de comutação canònica das coordenadas e seus momentos canonicamente conjugados, que só é válida se os vínculos foren de primeira classe em nível clássico.

Para encontrarmos a álgebra em nivel quântico necessitamos de umo ordenamento que permita tratar convenientemente funçöes do operador posiçäo $X$ de tal forma que:

$$
F(X):=F(X)
$$

Este ordenamento é justificado con uma escolha conveniente do vácuo da teoria escrevendo

$$
\begin{gathered}
X^{m \mu}=\frac{T}{2} \int_{0}^{x_{4}} d s e^{-2 i m \sigma} X^{\mu}=-\frac{i}{4} T \int d \xi \xi^{-m-1} X^{i \mu}(\xi)=\int_{m} X^{\mu}, \\
p_{\mu}^{m}=\int_{m} P_{\mu} \quad \xi=e^{2 i \sigma},
\end{gathered}
$$

onde os modos positivos e o modo zèro de $P^{m}$ são colocados à direita, os modos negativos e o modo zero de $X^{\mathrm{m}}$ sắo colocados a esquerda das expressôs escritas em funçăo de $X$ e $P$. Os produtos ordenados năo triviais são produtos de $X$ \& $P$, e.g.

$$
P_{\beta}(\sigma) X^{\nu}(\bar{\sigma})=: P_{\beta}(\sigma) X^{\nu}(\bar{\sigma}):+\underline{P_{\mu}(\sigma) X^{\nu}(\bar{\sigma})}
$$

com

$$
\underline{P_{\mu}(x) X^{\nu}(\bar{\omega})}=-\frac{i}{\pi} \delta_{\mu x}^{*} \frac{\bar{\xi}}{\xi-\xi}|\xi|<|\vec{\xi}|
$$

Uma relaçã̃o geral e importante é:

$$
\left[\int_{m} A_{2} \int_{n} B\right]=-\frac{T^{2}}{16} \int d \xi \int d \bar{\xi}^{-m-1} \bar{\xi}^{-n-1} A(\xi) B(\bar{\xi})
$$


Aplicando (4.96) e (4.97) aos vínculos usuais da corda, com o campo de fundo nâo degenerado, encontramos a algebra em nível quântico cujos termos adicionais, i.e., aqueles não proporcionais ans vínculos, também chamados de termos de Schwinger, se anulam se o campo de fundo satisfaz as equações de Einstein. Temos então que as equaçỏes de Einstein săo as condiçoes para a invariància conforme em nível quântico.

Com o estudo do método dessenvolvido em [32], podemos fazer o tratamento quântico da álgebra dos vínculos de primeira classe da corda num campo de fundo degenerado. Com isto, encontraremos os termos de Schwinger correspondentes à quebra dá invariância conforme e demais simetrias, tratadas quanticamente e estendidas para o caso degenerado.

É inportarte notarmos que ssta extensão envolvendo métricas degeneratlas não é possivel se o ponto de partida for a álgebra usual do caso näo degenerado. Isto porque o último caso, to admitir uma métrica inversa, permite a inclusāo de contra-termos, no tratamento em nivel quântico, proporcionais ao tensor de Riemann [31] e com isto inviabiliza uma extensăo direta para o caso degenerado. Vemos então que tal extensão deve partir do cálculo envolvendo a álgebra dos vínculos de primeira classe clescrita exclusivamente com a métrica degenerada.

O callculo em nivel quäntico ainda está em aberto, porém quando concluf́lo futuramente, poderá indicar um comportamento quântico para as métricas degeneradas consideradas no contexto da teoria de cordas. 


\section{Capítulo 5}

\section{Conclusão}

Os resultados obtidos nesta tese, revelam aspectos importantes da dinamica de partículas e cordas em espaços com métricas degeneradas que ainda näo foram completamente tratados na literatura. Neste trabalho conseguimos desenvolver o formalismo Hamiltoniano de forma a obter os vínculos e calcularmos a álgebra dos mesmos.

Ao desenvolvermos o formalismo Hamiltoniano de forma a obter o conjunto completo dos vínculos conseguimos determinar a dinamica efetiva para as partículas relativísticas e năo relativisticas e cordas em movimento num campo de fundo gravitacional degenerado. Verificamos que quando a métrica é degenerada há o surgimento de vínculos adicionais no espaço de fase os quais näo possuem correspondência no caso em que a métrica nâo é degenerada. Estes vinculos sâo caraeterísticas importantes para os movimentos em espaços com mêtricas degeneradas. A estrutura dos vineulos é semelhante para todos os modelos que tratamos. Porén, os vínculos apresentam maior complexidade quando há invariancia por reparametrizaçäo que caracteriza as partículas relativisticas e as cordas.

Um aspecto bastante motivador foi considerar a isometria no caso da particula não relativistica. Este modelo, por ser o caso mais simples envolvendo métrica degenerada, possibilitou uma análise simplificada da presença de isometrias associada à métrica degenerada. No caso usual em que a métrica nào é degenerada, uma isometria está associada 
a uma simetria intrínseca da métrica. Porém, no caso da corda com métrica degenerar da, concluimos que a isometria estát fortemente associada à simetria local gerada pelos vínculos de primeira classe. Isto mostra como o formalismo Hamiltoniano para a corda em campo de fundo degenerado revela aspectos geométricos que nào estavam explícitos na construção algêbrica dos vinculos de primeira classe. Convérn ressaltar que estes aspectos sâa exclusivos da corda com métrica degenerada que desenvolvemos nesta tese.

Ainda podemos notar que o uso da isometria para a métrica degenerada permite algumas motivações adicionais para o desenvolvimento de projetos futuros oriundos desta tese. Estas motivaçôes estão associadas à obtençăo de açês duais discutidas em [28, 29]. Estas açōes são assin definidas por serem classicamente equivalentes à ação oniginal de uma corda en campo de fundo gravitacional porán com métricas diferentes [30]. Um aspecto marcante nestas açōes duais é que para a sua construção não é necessário o inverso da métrica. Entäo, podemos analisar futuramente como as métricas degeneradas podem se relaçionar com as métricas obtidas nas açães duais.

Um trabalho importante para ser desenvolvido é o estudo de aspectos quânticos envolvendo métricas degeneradas. Conforme discutimos no final do Capítulo 4 , o desenvolvimento da álgebra em nivel quântico poderá fornecer outras aplicaçōes para a teoria de cordas em campo de fundo gravitacional degenerado. Este resultado poderá dar uma descrição alternativa para o estudo das dualidades em nivel quântico para as açôs duais. Isto se deve ao fato de que as isometrias, que são a base para esta simetria de dualidade, também estảo presentes na métrica degenerada. Assim, podemos esperar que exista uma relą̧ão especifica entre as métricas degeneradas e as não degeneradas no contexto da teoria de cordas. 


\section{Apêndice A}

\section{Propriedades dos Auto-Vetores Nulos em $D-r$ Ímpar}

Sabemos de $(3.26)$, que

$$
\Phi_{\underline{L}}=N_{\underline{\underline{z}}}+\lambda \lambda_{\underline{\underline{H}}}=0
$$

Porém, para $D-\vartheta$ impar, $M_{\text {世出 }}$ năo possui inversa, i.e., det $M=0$. Sendo assim, vamos considerar o indice $\underline{\mu}$ com a seguinte representação: $\underline{\mu}=(\underline{\mu}, \underline{D-r})$. Entäo, temos

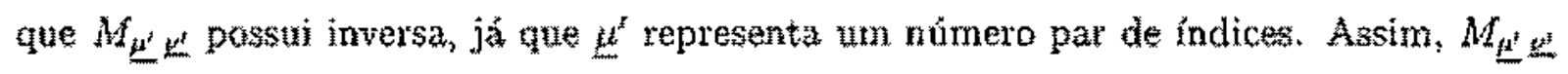
é de ordem par além de possuir componentes independentes, o que torna $\operatorname{det}\left(M_{{\underline{L^{\prime}}}^{\prime} \underline{u}^{\prime}}\right) \neq 0$. Desta forma podemos concluir que $M_{\text {सz }}$ tem posto $D-r-1$, possuindo apenas um auto-vetor mulo.

Seja $V H_{0}$ atutometor nulo, entäo

$$
V M_{H E}=0
$$

Multiplicando (A.I) por Vu, temos

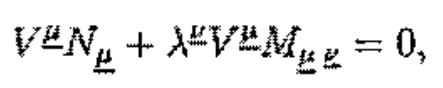

que implica em

$$
x \equiv Y \underline{Z} N_{\underline{L}}=0
$$


Entretanto, sabemos que

$$
\chi=N_{\underline{D-r}}-M_{\underline{D-r}} \underline{\beta}^{\prime} M^{\underline{\beta}^{\prime}} \underline{\underline{\nu}}^{\prime} N_{\underline{\underline{\nu}^{\prime}}}=0
$$

De (A.5) e (A.4), podemos fazer a seguinte identificação:

$$
V \underline{\underline{\mu}}=\delta_{\underline{D}-r}^{\underline{\underline{\mu}}}-M_{\underline{D-r}} \underline{\beta^{\prime}} M^{\underline{\beta^{\prime}}} \underline{\underline{\nu}}^{\prime} \delta_{\underline{\underline{\nu}}^{\prime}}^{\underline{\mu}}
$$

A partir de (A.6) podemos checar diretamente que

$$
\begin{aligned}
& V \underline{\underline{\mu}} M_{\underline{\mu} \underline{\underline{\mu}}}=M_{\underline{D-r} \underline{\underline{\nu}}}-M_{\underline{D-r}} \underline{\beta}^{\prime} M^{\underline{\beta}^{\prime} \underline{\underline{\alpha}}^{\prime}} M_{\underline{\alpha^{\prime}} \underline{\underline{\nu}}} \\
& =M_{\underline{D-r} \underline{\underline{u}}}-M_{\underline{D-r}} \underline{\underline{\rho}}^{\prime} \delta_{\underline{\underline{\beta}}}^{\underline{\beta}^{\prime}}=0 \text {. }
\end{aligned}
$$

Então podemos concluir que $V \underline{\underline{\mu}}$ é uma representação do auto-vetor com auto-valor nulo para qualquer que seja a ordem ímpar de $M_{\underline{\mu}} \underline{\underline{\nu}}$. Assim podemos escrever o vínculo $\chi$ de uma forma compacta, ou seja, $\chi=V \underline{\mu} N_{\underline{\mu}}$.

Uma observaçāo importante é que $V \underline{\mu}$ pode também ser construído diretamente a partir da equação de auto-valores. Consideremos o caso particular $(D-r)=3$ com a matriz associada a $M_{\underline{\mu}} \underline{\underline{\nu}}$ e um vetor em três dimensōes:

$$
\begin{gathered}
\mathbf{M}=\left[\begin{array}{ccc}
0 & M_{12} & M_{13} \\
-M_{12} & 0 & M_{23} \\
-M_{13} & -M_{23} & 0
\end{array}\right] \\
\mathrm{V}=\left(\begin{array}{c}
V^{1} \\
V^{2} \\
V^{3}
\end{array}\right)
\end{gathered}
$$

Consideremos as equaçōes para o auto-valor nulo de $\mathrm{M}$ :

$$
\mathbf{M} \cdot \mathbf{V}=0 \Longrightarrow\left\{\begin{array}{l}
V^{2} M_{12}+V^{3} M_{13}=0 \\
-V^{1} M_{12}+V^{3} M_{23}=0 \\
-V^{1} M_{13}+V^{2} M_{23}=0
\end{array}\right.
$$


A partir du (A.8) obtemos o auto-vetor de $M$ cujo auto-valor é nulo:

$$
V=V^{3}\left(\begin{array}{c}
\frac{M_{3 x}}{M_{3}} \\
-\frac{M_{3}}{M_{1}} \\
1
\end{array}\right)
$$

Vamos considerar simplesmente que

$$
V=\left(\begin{array}{c}
\frac{M_{22}}{M_{12}} \\
-\frac{M_{12}}{M_{12}} \\
1
\end{array}\right)
$$

Desta forma

$$
\mathrm{M} \cdot \mathrm{V}=\left(\begin{array}{c}
-M_{13}+M_{13} \\
-M_{23}+M_{23} \\
-\frac{M_{13} M_{23}}{M_{13}}+\frac{M_{28} M_{13}}{M_{13}}
\end{array}\right)=\left(\begin{array}{l}
0 \\
0 \\
0
\end{array}\right)
$$

que garante (A.10) como uma representacāo converiente do auto-vetor nulo de $\mathrm{M}$.

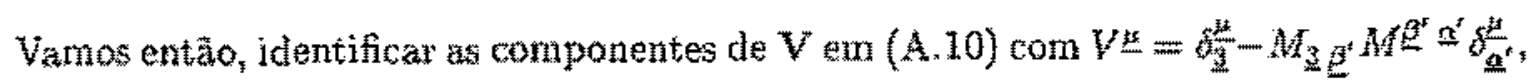
considerando que $(\underline{D-r})=3$ e $\alpha^{\prime}=(1,2)$.

Neste caso usaremos:

$$
M^{t}=\left(\begin{array}{cc}
0 & M_{12} \\
-M_{12} & 0
\end{array}\right) \Longrightarrow M^{*-1}=\left(\begin{array}{cc}
0 & -\frac{1}{M_{12}} \\
\frac{1}{M_{12}} & 0
\end{array}\right)
$$

onde devemos notar que $M$ é constituída apenas das componentes $M_{a^{\prime} E^{t}}$ e que estas componentes säo independentes e năo nulas, conforme vemos diretamente na matriz bidimensional anterior.

Ficamos antäo com

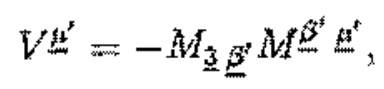

cujas componentes sào detalhadas a seguir.

Temos

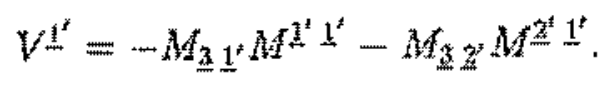


$\operatorname{Mas} M_{3} \underline{2}^{\prime}=-M_{23}=M_{32}$. Logo,

$$
V^{1^{\prime}}=-M_{32} \cdot \frac{1}{M_{12}}=\frac{M_{23}}{M_{12}},
$$

é o primeiro termo de $\mathrm{V}$ em (A.10). Considerando

$$
\begin{aligned}
V^{\underline{2}^{\prime}} & =-M_{\underline{3}} \underline{1}^{\prime} M^{1^{\prime} \underline{2}^{\prime}}-M_{\underline{3}}{\underline{2^{\prime}}}^{\prime} M^{\underline{2}^{\prime} \underline{2}^{\prime}} \\
& =-M_{31} \cdot\left(\frac{-1}{M_{12}}\right)=-\frac{M_{13}}{M_{12}},
\end{aligned}
$$

vernos que é o segundo termo de $\mathrm{V}$ em (A.10). E

$$
V^{\underline{D-r}}=V^{\underline{3}} \Rightarrow \delta_{\underline{3}}^{\frac{3}{3}}=1,
$$

é o terceiro termo de $\mathrm{V}$ em (A.10).

Devemos observar que os resultados obtidos são válidos para as partículas e para a corda bastando considerar os índices apropriados. 


\section{Apêndice B}

\section{Dedução da Hamiltoniana Canônica da Corda com Métrica Degenerada e Vínculos Secundários}

\section{Hamiltoniana Canônica}

Vamos considerar a Lagrangeana na formulação de Polyakov:

$$
\begin{aligned}
& L=\int C d \sigma \\
& L=-\frac{T}{2} \sqrt{-h h^{(\alpha)}(b)} g_{(a)} X^{\mu} \partial_{0)} X^{\nu} \sigma_{p u}
\end{aligned}
$$

onde $(a)=0,1$. A densidade de momento é dada por:

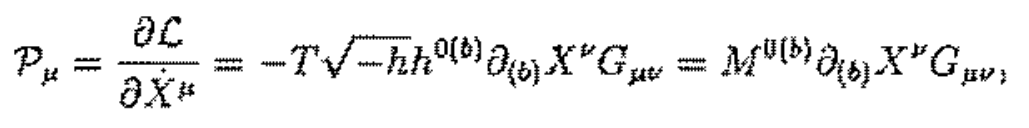

onde

$$
\partial_{0} X^{\mu}=\dot{X}^{\mu} \quad \partial_{1} X^{\mu}=X^{\prime \mu}
$$

Para $G_{p k}=e_{\mu}^{a^{2}} e_{p}^{b} \eta_{a^{\prime} b}$, sabanos que $\operatorname{det}\left(G_{\mu \mu}\right)=0$, já que o posto de $G$ é $r$. 
Vamos escrever $P_{k}$ como

$$
\begin{aligned}
p_{p} & =\Delta_{a^{*}} e_{a}^{a^{\prime}}, \\
\Delta_{a^{*}} & =M^{0(b)} \partial_{(b)} X^{*} e_{y}^{b^{\prime}} \eta_{a^{\prime},} .
\end{aligned}
$$

Vamos considerar que $\mu=\mu^{\prime}, \underline{\mu}$, onde $z^{z}=0,1, \ldots, r-1 ; \underline{\mu}=x, \ldots, D-1$. Então escrevemos (B.4) cono

$$
\begin{aligned}
& P_{\mu^{\prime}}=\Delta_{a^{t}} e_{\mu^{\prime}}^{a^{a^{\prime}}} \\
& P_{\underline{\underline{z}}}=\Delta_{a^{\prime}} e_{\underline{\underline{\mu}}}^{a^{t}}
\end{aligned}
$$

Mas:

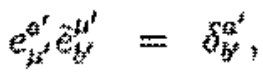

$$
\begin{aligned}
& e_{\mu^{\prime}}^{a^{z}} \dot{e}_{a^{z}}^{z^{z}}=g_{y^{\prime}}
\end{aligned}
$$

De (B.5) temos que

$$
\Delta_{a^{t}}=\tilde{e}_{a^{t}}^{k^{t}} p_{k^{t}}
$$

Usando esta última em (B.6), temos

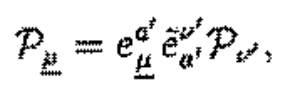

que corresponde ao vínculo primário

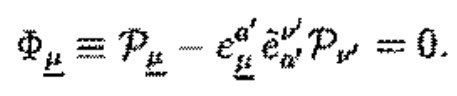

Vamos considerar que

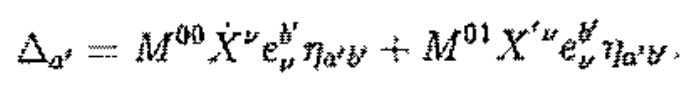


Entas

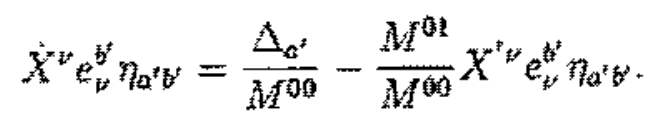

A partix clesta última, temos

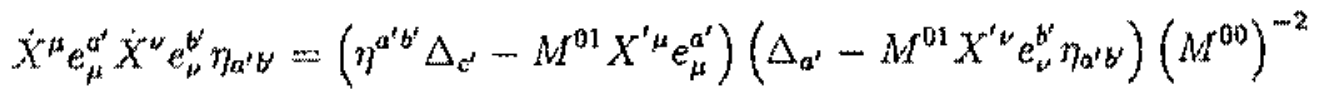

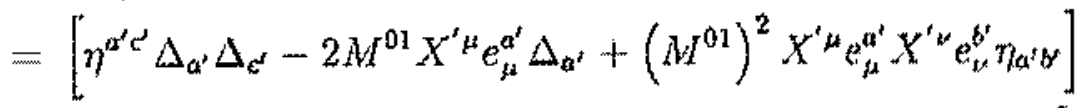

$$
\begin{aligned}
& \times\left(M^{00}\right)^{-2}
\end{aligned}
$$

Sabendo que

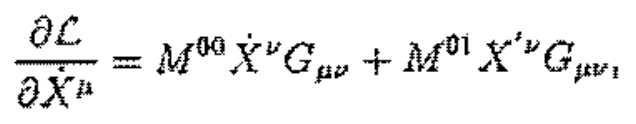

obtemos

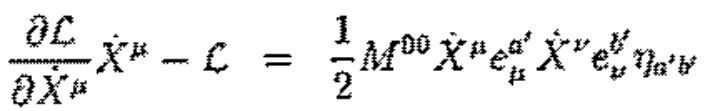

$$
\begin{aligned}
& -\frac{1}{2} M^{11} X^{s} e_{j}^{a^{*}} X^{*} e_{w}^{*} n_{a^{*} w^{*}}
\end{aligned}
$$

Ao considerarmos (B.9), obtemos as seguintes propriedades.

$$
\begin{aligned}
& \eta^{a^{\prime} k^{\prime}} \Delta_{\alpha^{\prime}} \Delta_{c^{\prime}}=\eta^{a^{\prime} c^{\prime}} \hat{e}_{a^{\prime}}^{\mu^{\prime}} \tilde{e}_{c^{\prime}}^{y^{\prime}} \mathcal{P}_{\mu^{\prime}} \mathcal{P}_{y^{\prime}}=\hat{G}^{\mu^{\prime} \nu^{\prime}} \mathcal{P}_{\mu^{\prime}} \mathcal{P}_{y^{\prime}} \\
& X^{\mu^{\prime} \mu} e_{e_{\mu}^{\prime}}^{\alpha^{\prime}} \Delta_{a^{\prime}}=X^{\prime \mu} \mathcal{P}_{\mu}
\end{aligned}
$$

Substituindo $(B .17)$ em $(B .14)$ e $(B .16)$, temos

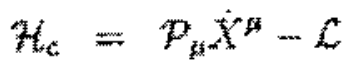

$$
\begin{aligned}
& =\frac{\tilde{P}^{2}}{2 M^{00}}-\frac{M^{01}}{M^{01}} P_{13} X^{*}+\left[\frac{\left(M^{01}\right)^{2}}{M^{00}}-M^{n}\right] \frac{\left(X^{n}\right)^{2}}{2}
\end{aligned}
$$


onde

$$
\begin{aligned}
& \bar{p}^{2}=\tilde{G}^{\mu^{2} w^{2}} p_{\mu^{*}} P_{w^{2}} \\
& \left(X^{1}\right)^{2}=X^{2} b X^{\prime}{ }^{\prime} G_{\mu \nu}
\end{aligned}
$$

Adicionalmente, sabemos que

$$
\operatorname{det}\left(h^{(a)(5)}\right)=\frac{1}{h}=h^{00} h^{\mathrm{u}}-\left(h^{01}\right)^{2}
$$

onde $h=\operatorname{det}\left(h_{(a)(b)}\right)$ e $h<0$. Considerando que $M^{(a)(b)}=-T \sqrt{-h} h^{(a)(b)}$ temos a partir de (B.10):

$$
\frac{\left(M^{01}\right)^{2}}{M^{00}}-M^{11}=-\frac{T}{\sqrt{-h h^{00}}}
$$

Substituindo estas propriedades em (B.18), ficamos com a densidade de Hamiltoniana canônica dada por:

$$
\mathcal{H}_{c}=-\frac{1}{2 h^{00}}\left[\frac{\tilde{p}^{2}}{T \sqrt{-h}}+2 h^{\mathrm{n} t} p_{\mu} X^{{ }^{\mu}}+\frac{T}{\sqrt{-h}}\left(X^{\prime}\right)^{2}\right]
$$

Devemos notar que quando a métrica năo for degenerada, basta substituirmos $\tilde{p}^{2}$ por $\mathcal{P}^{2}=G^{\mu *} \mathcal{P}_{\mu} \mathcal{P}_{k}$ para obtermos a forma padrão de $\mathcal{H}_{6}$ do caso não degenerado.

\section{Vínculos Secundários}

Na evolução dos vínculos primários $\mathcal{P}_{\{a)(b)}=0$ através da Hamiltoniana primária. $H_{P}$, temos que considerar as contribuiçōes vindas do cada componente, ou seja, precisamos calcular $\dot{\mathcal{P}}_{00}, \dot{\mathcal{P}}_{0 \mathrm{y}}$ e $\dot{\mathcal{P}}_{11}$. Assim temos 


$$
\dot{\mathcal{P}}_{(\sigma)(d)}(\sigma)=\left\{\mathcal{P}_{(\sigma)(d)}\left(\sigma^{\prime}\right), H_{p}\right\}=\int_{0}^{\sigma_{1}}\left(\left\{\mathcal{P}_{(s)(d)}(\sigma), \mathcal{H}_{c}\left(\sigma^{\prime}\right)\right\}\right) d \sigma^{\prime}
$$

onde $\mathcal{H}_{c}$ estą definida em (B.21) e também usamos que

$$
\begin{array}{r}
\left\{p_{(\sigma)(d)}(\sigma), P_{(a)(b)}\left(\sigma^{n}\right)\right\}=0 \\
\left\{P_{(\sigma)(d)}(\sigma), \Phi_{\underline{u}}\left(\sigma^{5}\right)\right\}=0
\end{array}
$$

A contribuiçäo em (B.22) é dada por

$$
\begin{aligned}
& \left\{\mathcal{P}_{(\mathrm{c})\left\{\alpha(\sigma), \mathcal{H}_{c}\left(\sigma^{\prime}\right)\right\}=}\right. \\
& \left\{\mathcal{P}_{(c)(\alpha)}(\sigma),-\frac{1}{2 h^{00}\left(\sigma^{\prime}\right)}\right\}\left[\frac{\tilde{p}^{2}}{T \sqrt{-h}}+2 h^{01} \mathcal{P}_{\mu} X^{\prime \mu}+\frac{T}{\sqrt{-h}}\left(X^{\prime}\right)^{2}\right]\left(\sigma^{\prime}\right) \\
& -\frac{1}{2 h^{00}\left(\sigma^{\prime}\right)}\left\{\mathcal{P}_{(c)(d)}(\sigma),\left[\frac{\overline{\mathcal{P}}^{z}}{T \sqrt{-h}}+2 h^{0 \mathrm{i}} \mathcal{P}_{\mu} X^{\prime \mu}+\frac{T}{\sqrt{-h}}\left(X^{\prime}\right)^{2}\right]\left(\sigma^{\prime}\right)\right\}
\end{aligned}
$$

Em (B.25) vamos considerar o termo

$$
\begin{aligned}
& \left\{P_{(o)}(a),\left[\frac{\bar{p}^{2}}{T \sqrt{-h}}+2 \hbar^{(\omega)} \mathcal{P}_{\mu} X^{\prime \mu}+\frac{T}{\sqrt{-h}}\left(X^{\prime}\right)^{2}\right]\left(\sigma^{\prime}\right)\right\}= \\
& \left\{P_{(\sigma) d d}(\sigma), \frac{1}{\sqrt{-h}}\left(\sigma^{n}\right)\right\}\left(\frac{\bar{p}^{2}}{T}\left(\sigma^{\prime}\right)+T\left(X^{\prime}\right)^{2}\left(\sigma^{n}\right)\right)
\end{aligned}
$$

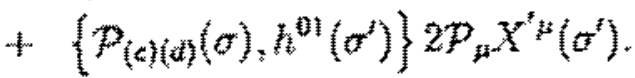

No termo (B.26) precisamos calcular $\left\{p_{(c)(d)}(\sigma), \frac{1}{\sqrt{-4}}\left(\sigma^{\prime}\right)\right\}$. Porém, vamos considerar

$$
\left\{p_{(c)\} d\rangle}(\sigma), f\left(\sigma^{\prime}\right)\right\}=-\delta_{(c)\}(d)}^{(a)(b)} \delta\left(\sigma-\sigma^{n}\right) \frac{\partial f\left(\sigma^{\prime}\right)}{\partial h^{(a)(b)}\left(\sigma^{\prime \prime}\right)}
$$

Mas, para $f(\sigma)=(-h)^{-1 / 2}(\sigma)$, temos 


$$
\frac{\partial f\left(\sigma^{\prime}\right)}{\partial h_{(b)(b)}\left(\sigma^{\prime \prime}\right)}=-\frac{1}{2}(-h)^{-1 / 2}\left(\sigma^{\prime}\right) h_{(a)(b)}\left(\sigma^{\prime}\right) \delta\left(\sigma^{\prime} \cdots \sigma^{t \prime}\right) .
$$

Então, substituindo (B.28) en (B.27), ficamos com

$$
\left\{\mathcal{P}_{(c)(d)}(\sigma), \frac{1}{\sqrt{-h}}\left(\sigma^{\prime}\right)\right\}=-\frac{1}{2}(-h)^{-1 / 2}\left(\sigma^{\prime}\right) h_{(c)(\alpha)}\left(\sigma^{\prime}\right) \delta\left(\sigma-\sigma^{\prime}\right)
$$

Un outro parênteses de Poisson em (B.25) é dado por

$$
\begin{aligned}
& \left\{P_{(c)(d)}(\sigma),-\frac{1}{2 h^{00}\left(\sigma^{7}\right)}\right\}=-\frac{1}{2} \delta_{(c)\}(d)}^{(a)(b)} \frac{\partial\left(-\frac{1}{\left.h^{00}\right)}\right)}{\partial h^{(a)(b)}} \\
& =\frac{1}{2} \delta_{(0)(t)}^{00}\left(h^{00}\right)^{-2}\left(\sigma^{\prime}\right) \delta\left(\sigma-\sigma^{\prime}\right) \text {, }
\end{aligned}
$$

ande $\delta_{\langle c\rangle(d)}^{(a)(d)}=\frac{1}{2}\left(b_{(e)}^{(a)} \delta_{(d)}^{(b)}+\delta_{(c)}^{(b)} \delta_{(d)}^{(a)}\right)$

Entäo, usando (B.30) e (B.29), ficamos com (B.25) dada por

$$
\begin{aligned}
& \left\{P_{(c)(d)}(\sigma), H_{c}\left(\sigma^{\prime}\right)\right\}=\frac{1}{2} \delta_{(\omega)\{d\}}\left(h^{00}\right)^{-2}\left(\sigma^{\prime}\right)\left[\frac{p^{2}}{T}+T X^{\prime 2}+P_{\mu} X^{\prime \mu}\right]\left(\sigma^{\prime}\right) \delta\left(\sigma-\sigma^{\prime}\right) \\
& +\frac{1}{2} \frac{1}{h^{00}\left(\sigma^{\prime}\right)}(-h)^{-1 / 2}\left(\sigma^{\prime}\right) h_{(c)(\alpha)}\left(\sigma^{2}\right)\left[\frac{\tilde{p}^{2}}{T}+T X^{2}\right] \delta\left(\sigma-\sigma^{r}\right)
\end{aligned}
$$

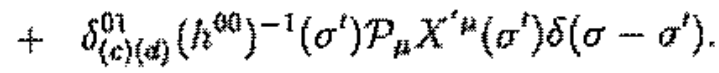

A partir de (B.31), temos em $(B, 22)$

$$
\begin{aligned}
\dot{\mathcal{P}}_{01}(\sigma)= & \dot{\mathcal{P}}_{10}(\sigma)=\frac{1}{2} \frac{h^{01}}{h^{00}}(-h)^{-1 / 2}(\sigma)\left[\frac{\tilde{p}^{2}}{T}+T X^{\prime 2}\right](\sigma) \\
& +\frac{1}{2} \frac{1}{h^{00}(\sigma)} \mathcal{P}_{\mu} X^{\prime \mu}(\sigma)=0 \\
\dot{P}_{00}(\sigma) & =\frac{1}{2} \frac{1}{\left(h^{00}\right)^{2}(\sigma)}\left[\frac{\tilde{p}^{2}}{T}+T X^{x^{2}}+\mathcal{P}_{\mu} X^{\prime \mu}\right](\sigma) \\
& +\frac{1}{2}(-h)^{-1 / 2}(\sigma) \frac{h_{00}}{h^{00}}\left[\frac{\tilde{p}^{2}}{T}+T X^{x_{2}}\right](\sigma)=0
\end{aligned}
$$




$$
\begin{aligned}
\dot{p}_{1}(\sigma) & =\frac{1 h_{11}}{2} \frac{h^{00}}{}(-h)^{-1 / 2}(\sigma)\left[\frac{\tilde{p}^{2}}{T}+T X^{\prime 2}\right](\sigma) \\
& =\frac{1 h_{11}}{2}(-h)^{-1 / 2}(\sigma)\left[\frac{\bar{p}^{2}}{h^{2}}+X^{x^{2}}\right](\sigma)=0
\end{aligned}
$$

Camo $h_{11} \neq 0, h_{00} \neq 0$ e $h \neq 0$, (B.34) é equivalente a

$$
\frac{\tilde{P}^{2}}{T}(\sigma)+T X^{2}(\sigma)=0
$$

Usando (B.35), vemos que (B.32) e (B.33) se reduzem apenas a

$$
\frac{1}{2 h^{00}} \mathcal{P}_{p} X^{*}(\theta)=0
$$

Entz̃o, multíplicando (B.35) e (B.36) por $T^{-1}$ a sabendo que $h^{\text {to }} \neq 0 \mathrm{em}(\mathrm{B} .30)$, ternos os vínculos secundários

$$
\begin{aligned}
& \varphi_{1} \equiv \frac{1}{T} p_{\mu} X^{\mu}=0 \\
& \varphi_{2} \equiv \frac{\tilde{p}^{2}}{T^{2}}+X^{x_{2}}=0
\end{aligned}
$$




\section{Apêndice C}

\section{Dedução da Álgebra dos Vínculos para a Corda}

Neste apèndice vamos calcular alguns parênteses de Poisson envolvendo os vínculos da corda con métrica degenerada e utilizados no Capítulo 4.

A seguir, temos os parênteses de Poisson envolvendo os vínculos $P_{(a)(b)}$ com as demais vinculos, $\Phi_{\underline{\mu}}, \varphi_{1}$ e $\varphi_{2}$ que năo dependem de $h^{(\mathfrak{a})(b)}$, encontrados no Capitulo 4 .

$$
\begin{aligned}
& \left\{P_{(a)(b)}, \Phi_{13}\right\}=0, \\
& \left\{\mathcal{P}_{\{a \mid(b)}, \varphi_{l}\right\}=0=\left\{\mathcal{P}_{\left.(a)\}(b), \varphi_{2}\right\}}\right\}
\end{aligned}
$$

Para os vínculos $\varphi_{1}$ e $\Phi_{\underline{L}}$ temos

$$
\begin{aligned}
& \left\{\varphi_{1}(\sigma), \Phi_{\mathrm{g}}\left(\sigma^{\prime}\right)\right\}=p_{\mu}(\sigma)\left\{X^{\prime \mu}(\sigma), P_{\mathrm{p}}\left(\sigma^{\prime}\right)\right\}
\end{aligned}
$$

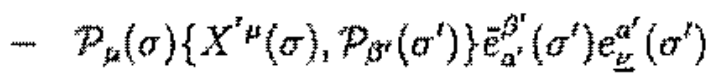

$$
\begin{aligned}
& +X^{\prime \mu}(\sigma) \mathcal{P}_{\beta^{t}}\left(\sigma^{t}\right)\left\{\mathcal{P}_{\mu}(\sigma), \hat{e}_{z^{*}}^{z^{*}} e_{\mathrm{z}}^{a^{t}}\left(\sigma^{\prime}\right)\right\}
\end{aligned}
$$

Na equaçäo (C.3), podemos considerar o seguinte termo:

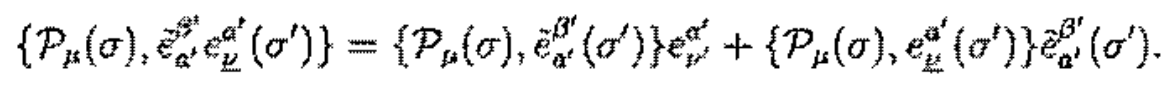


Max,

$$
\begin{aligned}
\left\{f\left(X\left(\sigma^{f}\right)\right), p_{\mu}(\sigma)\right\}_{r-q^{*}} & =\partial_{\mu}\left(\sigma^{*}\right) f\left(X\left(\sigma^{\prime}\right)\right) \delta\left(\sigma-\sigma^{\prime}\right) \\
\left(X^{t}\left(\sigma^{\prime}\right), p_{\mu}(\sigma)\right)_{r+\psi^{*}} & =\delta_{\mu}^{\nu} \delta\left(\sigma \cdots \sigma^{\prime}\right)
\end{aligned}
$$

Logo,

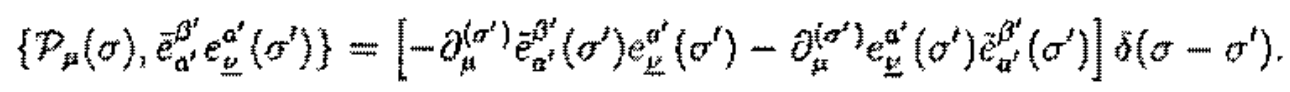

Também temos, partir de (C.5),

$$
\left\{X^{\prime} \mu(\sigma), P_{\underline{t}}\left(\sigma^{\prime}\right)\right\}=\delta_{\underline{\underline{z}}}^{\mu} \partial_{\sigma} \delta\left(\sigma-\sigma^{\prime}\right)
$$

Entăo, (C.3) equivale a

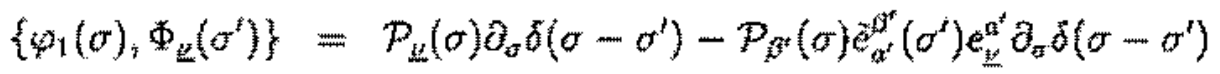

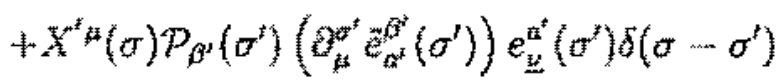

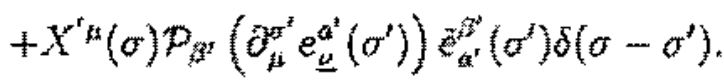

Porém, o segundo termo de (C.9) pode ser escrito como

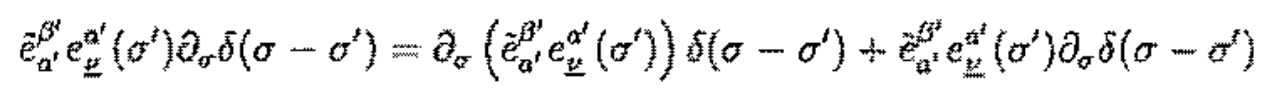

Logo,

$$
\begin{aligned}
& \left\{\varphi_{1}(\sigma)_{*}{ }_{w}\left(\sigma^{\prime}\right)\right\}=
\end{aligned}
$$

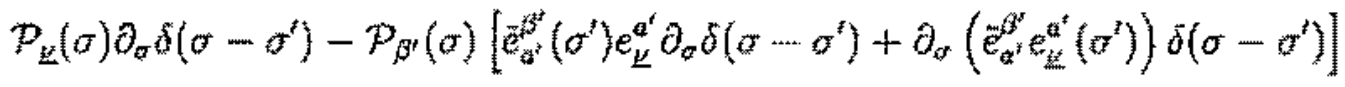

$$
\begin{aligned}
& +X^{i}(\sigma) \rho_{p r} \partial_{\sigma}\left(e_{\alpha^{\prime}}^{\beta^{\prime}} e^{a^{*}}\left(\sigma^{*}\right)\right) \delta\left(\sigma-\sigma^{\prime}\right)
\end{aligned}
$$


Ao usamos $\mathrm{em}(\mathrm{C} .11)$, a seguinte propriedade,

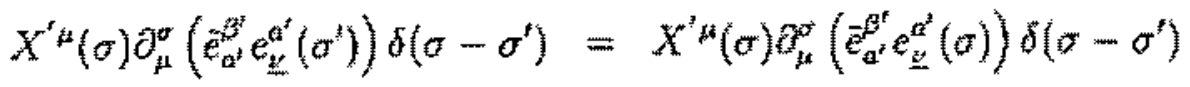

$$
\begin{aligned}
& =\partial_{\sigma}\left(e_{a^{\prime}}^{a^{\prime}} e_{\underline{\underline{L}}}^{a^{\prime}}(\sigma)\right)
\end{aligned}
$$

ficamos finalmente com

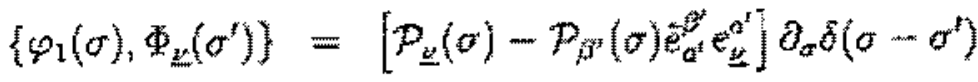

$$
\begin{aligned}
& =\Phi_{\underline{\nu}}(\sigma) \partial_{\sigma} \delta\left(\sigma-\sigma^{\prime}\right) \text {. }
\end{aligned}
$$

Consideremos agora o parenteses de Poisson entre os vinculos $\Phi_{t}$ e

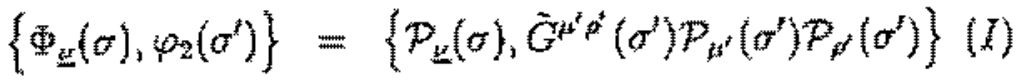

$$
\begin{aligned}
& +\left\{\mathcal{P}_{\underline{L}}(\sigma), X^{+\mu}\left(\sigma^{\prime}\right) X^{\mu^{\prime}}\left(\sigma^{\prime}\right) G_{\mu \rho}\left(\sigma^{\prime}\right)\right\}(I I)
\end{aligned}
$$

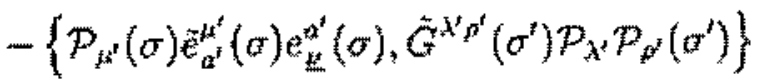

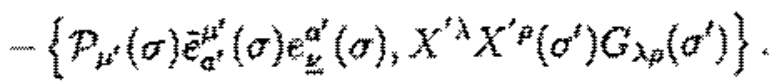

Vamos analisar cada termo. $O$ termo (I) de (C.14) é dado por

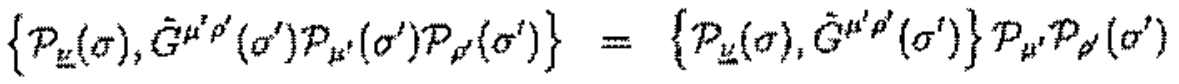

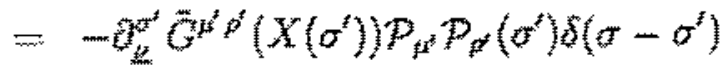

O tamo (II) de (C.14) e dado por

$$
\begin{aligned}
& \left\{\mathcal{P}_{\mu}(\sigma), X^{\prime \mu}\left(\sigma^{\prime}\right) X^{\prime}{ }^{\prime}\left(\sigma^{\prime}\right) G_{\mu \rho}\left(\sigma^{\prime}\right)\right\}=2\left\{\mathcal{P}_{\underline{L}}(\sigma), X^{\prime \mu}\left(\sigma^{\prime}\right)\right\} X^{\prime p}\left(\sigma^{\prime}\right) G_{\mu \rho}\left(\sigma^{\prime}\right)
\end{aligned}
$$

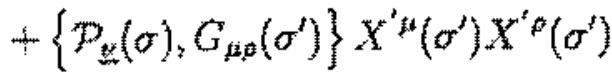

$$
\begin{aligned}
& =2 X^{*}\left(\sigma^{n}\right) G_{z y}\left(\sigma^{n}\right) \partial_{g} \delta\left(\sigma-\sigma^{3}\right)
\end{aligned}
$$

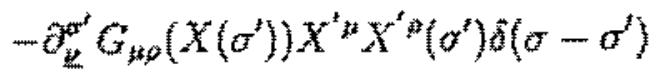


O termo (III) de (C.14) é dado por

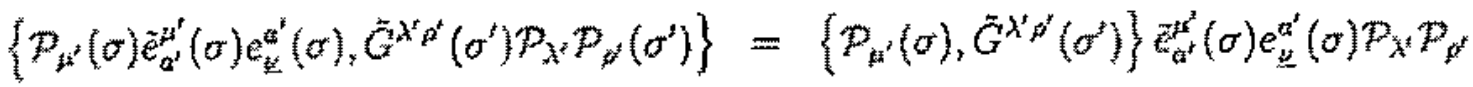

$$
\begin{aligned}
& +P_{\mu^{\prime}}(\sigma)\left\{\ddot{e}_{a^{\prime}}^{\mu^{\prime}}(\sigma) e_{\underline{\underline{g}}}^{a^{\prime}}(\sigma), P_{X^{\prime}} P_{\mu}\left(\sigma^{\prime}\right)\right\} \\
& \times \bar{G}^{i} p^{\prime}\left(\sigma^{\prime}\right) \text {, }
\end{aligned}
$$

onde

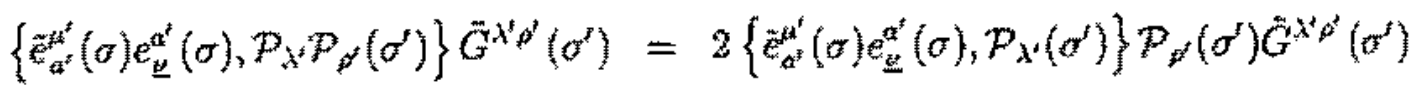

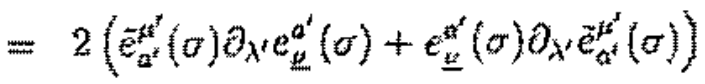

$$
\begin{aligned}
& \times P_{\beta^{\prime}}\left(\sigma^{\prime}\right) \hat{G}^{\lambda^{\prime} p^{\prime}}\left(\sigma^{\prime}\right) \delta\left(\sigma-\sigma^{\prime}\right) \text {. }
\end{aligned}
$$

Então, o lado direito da equą̧ão (C.17) vale

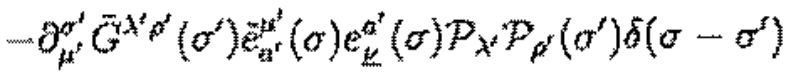

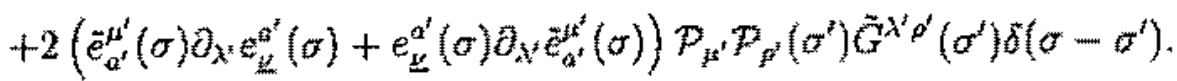

O termo (V) de (C.14) edado por

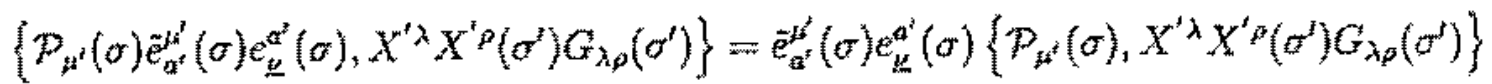

$$
\begin{aligned}
& =\hat{e}_{a^{\prime}}^{\mu^{\prime}}(\sigma) \varepsilon_{\underline{\underline{a}}}^{a^{\prime}}(\sigma)\left(2\left\{\mathcal{P}_{\mu^{\prime}}(\sigma), X^{\prime \lambda}\left(\sigma^{\prime}\right)\right\} X^{\prime}\left(\sigma^{\prime}\right) G_{\lambda \rho}\left(\sigma^{\prime}\right)+\left\{\mathcal{P}_{\mu^{\prime}}(\sigma), G_{\lambda \rho}\left(\sigma^{\prime}\right)\right\} X^{\prime \lambda}\left(\sigma^{\prime}\right) X^{x} \rho\left(\sigma^{\prime}\right)\right) \\
& =2 \hat{e}_{a^{t}}^{a^{t}}(\sigma) e_{\underline{u}}^{a^{t}}(\sigma) X^{\prime} \rho\left(\sigma^{t}\right) G_{p^{t} \rho} \partial_{\sigma} \delta\left(\sigma-\sigma^{\prime}\right)
\end{aligned}
$$

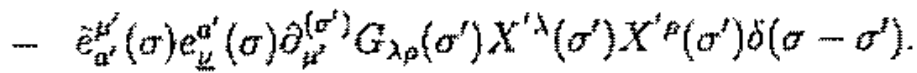

Vamos reagrupar os termos anteriormente descritos, de modo que possamos simplificar $\left\{\Phi_{\underline{w}}, \phi_{2}\right\}$. Com isto, ficamos com 


$$
\begin{aligned}
& \left\{\Phi_{\mathrm{k}}(\sigma), \sigma_{2}\left(\sigma^{r}\right)\right\}=
\end{aligned}
$$

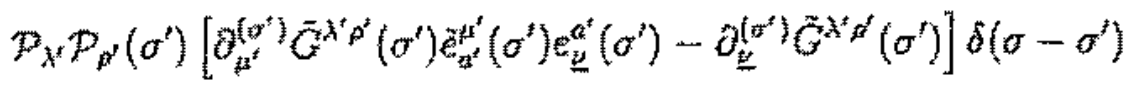

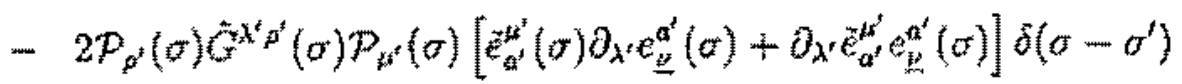

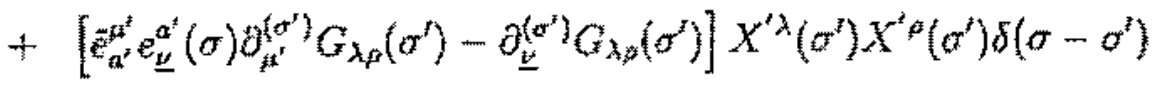

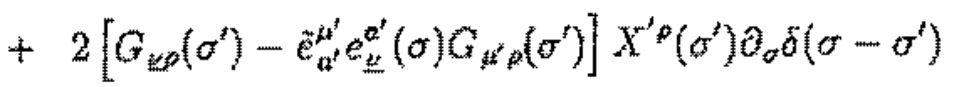

Considerando o termo (a), temos que

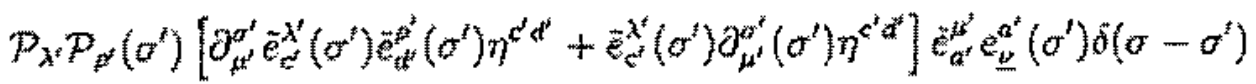

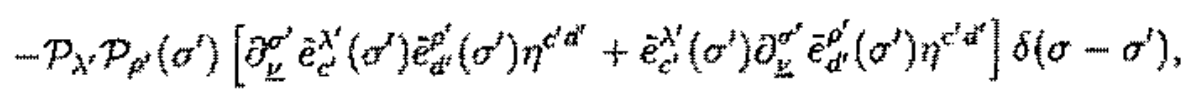

que é equivalente a

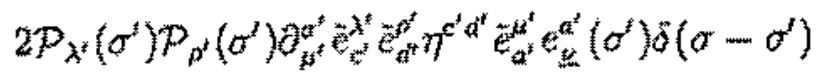

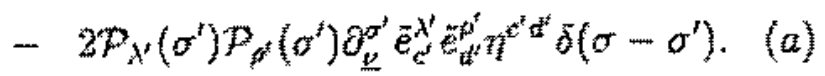

Vamos considerar nos termos (a) e (b) que

$$
\partial_{\mu} e_{e}^{\lambda^{\prime}}=-\tilde{e}_{e^{\prime}}^{p^{*}} e_{b^{\prime}}^{\lambda^{z}} \partial_{\mu} e_{p^{\prime}}^{b^{\prime}}
$$

Então a soma dos termos (a) e (b) é dada por:

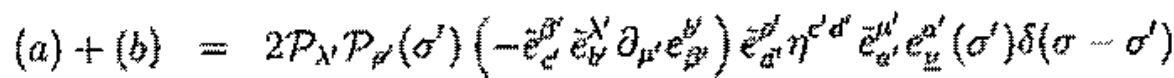

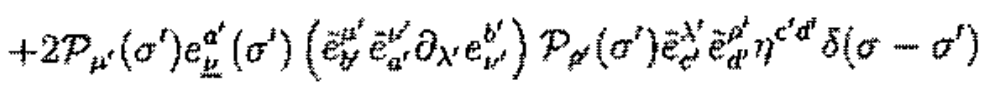

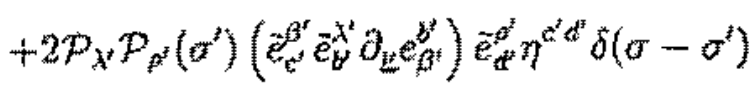

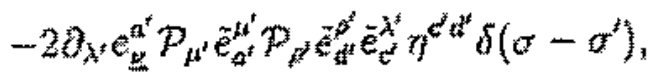


que reagrupando os termos acima, resulta em

$$
\begin{aligned}
& (a)+(b)=2\left(\partial_{\underline{\nu}} e_{\lambda^{\prime}}^{a^{\prime}}-\partial_{\lambda^{\prime}} e_{\underline{\underline{a}}}^{a^{\prime}}\right) \mathcal{P}_{\mu^{\prime}} \tilde{e}_{a^{\prime}}^{\mu^{\prime}} \mathcal{P}_{\rho^{\prime}} \tilde{e}_{d^{\prime}}^{\rho^{\prime}} \tilde{c}_{c^{\prime}}^{\lambda^{\prime}} \eta^{c^{\prime} d^{\prime}} \delta\left(\sigma-\sigma^{\prime}\right) \\
& +2\left(\partial_{\nu^{\prime}} e_{\lambda^{\prime}}^{b^{\prime}}-\partial_{\lambda^{\prime}} e_{\nu^{\prime}}^{b^{\prime}}\right) \mathcal{P}_{\mu^{\prime}} \tilde{e}_{\underline{\mu}}^{a^{\prime}} \mathcal{P}_{\rho^{\prime}} e_{d^{\prime}}^{\bar{\rho}^{\prime}} e_{a^{\prime}}^{\lambda^{\prime}} e_{\gamma^{\prime}}^{\mu^{\prime}} \tilde{e}_{c^{\prime}}^{\nu^{\prime}} \eta^{c^{\prime} d^{\prime}} \delta\left(\sigma-\sigma^{\prime}\right) \\
& =2 \tilde{\mathcal{P}}_{a^{\prime}} \overline{\mathcal{P}}_{d^{\prime}} \bar{e}_{c^{\prime}}^{\lambda^{\prime}} \eta^{c^{\prime} d^{\prime}} \partial_{[\underline{L}} e_{\left.\lambda^{\prime}\right]}^{a^{\prime} \delta} \delta\left(\sigma-\sigma^{\prime}\right) \\
& +2 \tilde{\mathcal{P}}_{b^{\prime}} \tilde{\mathcal{P}}_{d^{\prime}} \hat{e}_{c^{\prime}}^{\beta^{\prime}} \eta^{c^{\prime} d^{\prime}} e_{\underline{\underline{\nu}}}^{a^{\prime}} \tilde{e}_{a^{\prime}}^{\lambda^{\prime}} \partial_{\left[\beta^{\prime}\right.} e_{\left.\lambda^{\prime}\right]}^{a^{\prime}} \delta\left(\sigma-\sigma^{\prime}\right)
\end{aligned}
$$

Ao considerarmos o termo (c), devemos notar que:

$$
\begin{aligned}
(c) & =\left(e_{\underline{\nu}}^{a^{\prime}} e_{a^{\prime}}^{\mu^{\prime}}\left(\sigma^{\prime}\right) \partial_{\mu^{\prime}}^{\left(\sigma^{\prime}\right)} G_{\lambda \rho}\left(\sigma^{\prime}\right)-\partial_{\underline{\nu}}^{\left(\sigma^{\prime}\right)} G_{\lambda \rho}\left(\sigma^{\prime}\right)\right) X^{\prime \lambda} X^{\prime} \rho(\sigma) \delta\left(\sigma-\sigma^{\prime}\right) \\
& =-\mathcal{U}_{\underline{\nu}}^{\mu}(\sigma) \partial_{\mu}^{\left(\sigma^{\prime}\right)} G_{\lambda \rho}\left(\sigma^{\prime}\right) X^{\prime \lambda} X^{\prime} \rho(\sigma) \delta\left(\sigma-\sigma^{\prime}\right)
\end{aligned}
$$

onde

$$
\mathcal{U}_{\underline{\underline{L}}}^{\mu}=\delta_{\underline{\underline{u}}}^{\mu}-e_{\underline{\underline{\alpha}}}^{\boldsymbol{\alpha}^{\prime}} \bar{e}_{a^{\prime}}^{\chi^{\prime}} \delta_{\chi^{\prime}}^{\mu} \quad \text { e } \quad \mathcal{U}_{\underline{\underline{\nu}}}^{\mu} G_{\mu \nu}=0
$$

Da mesma forma para o termo (d):

$$
\begin{aligned}
(d) & =2\left[G_{\underline{\nu} \rho}\left(\sigma^{\prime}\right)-\tilde{e}_{a^{\prime}}^{\mu^{\prime}}(\sigma) e_{\underline{\underline{a}}}^{a^{\prime}}(\sigma) G_{\mu^{\prime} \rho}\left(\sigma^{\prime}\right)\right] X^{\prime \rho}\left(\sigma^{\prime}\right) \partial_{\sigma} \delta\left(\sigma-\sigma^{\prime}\right) \\
& =2 \mathcal{U}_{\underline{\underline{\mu}}}^{\mu}(\sigma) G_{\mu \rho}\left(\sigma^{\prime}\right) X^{\prime} \rho\left(\sigma^{\prime}\right) \partial_{\sigma} \delta\left(\sigma-\sigma^{\prime}\right)
\end{aligned}
$$

Entāo

$$
\begin{aligned}
\left\{\Phi_{\underline{\nu}}(\sigma), \varphi_{2}\left(\sigma^{\prime}\right)\right\}= & 2 \tilde{\mathcal{P}}_{a^{\prime}} \overline{\mathcal{P}}_{d^{\prime}} \tilde{e}_{c^{\prime}}^{\lambda^{\prime}} \eta^{c^{\prime} d^{\prime}} \partial_{\underline{L}} e_{\lambda^{\prime} !}^{a^{\prime}} \delta\left(\sigma-\sigma^{\prime}\right) \\
& +2 \tilde{\mathcal{P}}_{b^{\prime}} \overline{\mathcal{P}}_{d^{\prime}} \tilde{e}_{c^{\prime}}^{\beta^{\prime}} \eta^{c^{\prime} d^{\prime}} e_{\underline{\underline{a}}}^{a^{\prime}} \tilde{e}_{a^{\prime}}^{\lambda^{\prime}} \partial_{\left[\beta^{\prime}\right.} e_{\left.\lambda^{\prime}\right]}^{b^{\prime}} \delta\left(\sigma-\sigma^{\prime}\right) \\
& -\mathcal{U}_{\underline{\underline{\nu}}}^{\mu}(\sigma) \partial_{\mu}^{\left(\sigma^{\prime}\right)} G_{\lambda \rho}\left(\sigma^{\prime}\right) X^{\prime \lambda} X^{\prime \rho}(\sigma) \delta\left(\sigma-\sigma^{\prime}\right) \\
& +2 \mathcal{U}_{\underline{\mu}}^{\mu}(\sigma) G_{\mu \rho}\left(\sigma^{\prime}\right) X^{\prime} \rho\left(\sigma^{\prime}\right) \partial_{\sigma} \delta\left(\sigma-\sigma^{\prime}\right) .
\end{aligned}
$$

77 
O parêtnteses de Poisson entre os vínculos $\Phi_{\underline{L}}$, considerados em pontos diferentes, 6 dado por

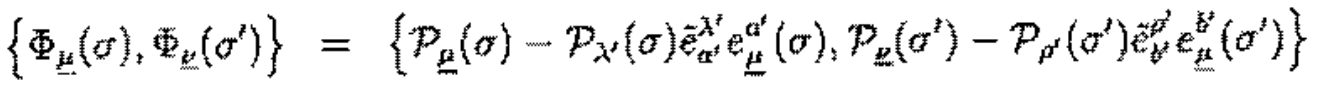

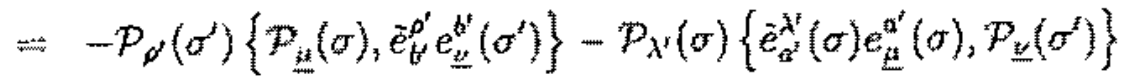

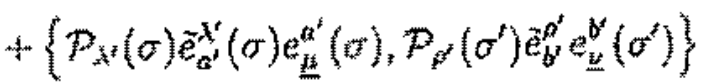

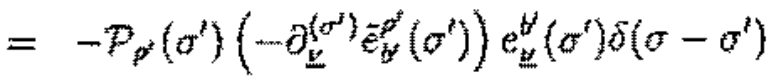

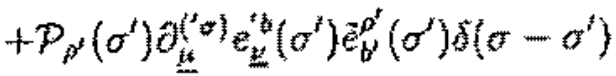

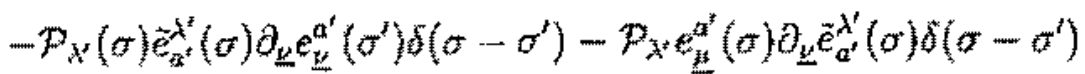

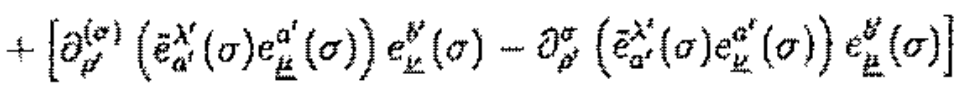

$$
\begin{aligned}
& \times P_{x^{\prime}}(\sigma) e_{b^{\prime}}^{\delta} \delta\left(\sigma-\sigma^{t}\right) \text {. }
\end{aligned}
$$

Reagrupando os termos de (C.32), finalmente temos

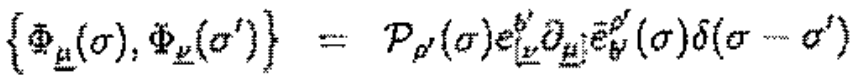

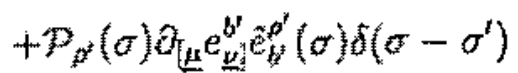

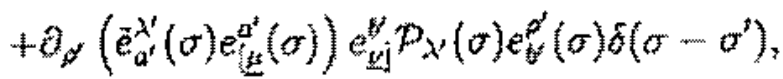

que apresenta uma forma similar à da partícula relativística.

Considerando o parênteses de Foisson entre os vínculos $\varphi_{1}$, temos

$$
\begin{aligned}
& \left\{\varphi_{1}(\sigma), \varphi_{1}\left(\sigma^{t}\right)\right\}=\mathcal{P}_{\mu}(\sigma)\left\{X^{t}{ }^{t}(\sigma), \mathcal{P}_{\nu}\left(\sigma^{\prime}\right)\right\} X^{\prime \mu}\left(\sigma^{\prime}\right) \\
& +X^{s / s}(\sigma) \mathcal{P}_{u}\left(\sigma^{\prime}\right)\left\{P_{\mu}(\sigma), X^{\prime \nu}\left(\sigma^{\prime}\right)\right\} \\
& =P_{\mu}(\sigma) X^{\prime \mu}\left(\sigma^{\prime}\right) \partial_{\sigma} g\left(\sigma-\sigma^{\prime}\right) \\
& \left.-\mathcal{P}_{\mu}\left(\sigma^{\prime}\right) X^{*}+\sigma\right) \partial_{\sigma^{\prime}} \delta\left(\sigma-\sigma^{\prime}\right) \text {. }
\end{aligned}
$$


Porén, vamos reescrever (C.34) utilizando as seguintes propriedades:

$$
\begin{aligned}
\partial_{\sigma}\left(X^{\prime \mu}\left(\sigma^{\prime}\right) \delta\left(\sigma-\sigma^{\prime}\right)\right) & =X^{\mu}\left(\sigma^{\prime}\right) \partial_{\sigma} \delta\left(\sigma-\sigma^{\prime}\right) \\
& =\partial_{\sigma}\left(X^{\prime \mu}(\sigma) \delta\left(\sigma-\sigma^{\prime}\right)\right) \\
& =X^{\prime \mu}(\sigma) \delta\left(\sigma-\sigma^{\prime}\right)+X^{\mu}(\sigma) \partial_{\sigma} \delta\left(\sigma-\sigma^{\prime}\right) \\
X^{\prime \mu}\left(\sigma^{\prime}\right) \partial_{\sigma} \delta\left(\sigma-\sigma^{\prime}\right) & =X^{\mu \prime}(\sigma)+X^{\prime \mu}(\sigma) \partial_{\sigma^{\prime}} \delta\left(\sigma-\sigma^{\prime}\right) \\
\partial_{\sigma^{\prime}} \delta\left(\sigma-\sigma^{\prime}\right) & =-\partial_{\sigma} \delta\left(\sigma-\sigma^{\prime}\right)
\end{aligned}
$$

Usando (C.35) a (C.37) em (C.34), fieamos com

$$
\begin{aligned}
& \mathcal{P}_{k}(\sigma)\left[X^{\prime \mu}(\sigma) \delta\left(\sigma-\sigma^{\prime}\right)-X^{\prime \prime}(\sigma) \partial_{\sigma} \delta\left(\sigma-\sigma^{\prime}\right)\right]
\end{aligned}
$$

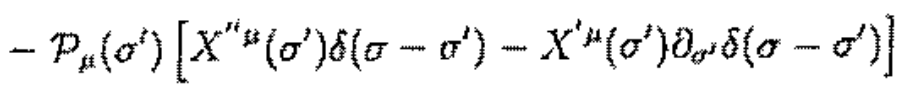

$$
\begin{aligned}
& =\mathcal{P}_{\mu}(\sigma) X^{*}(\sigma) \partial_{\sigma} \delta\left(\sigma-\sigma^{\prime}\right)+\mathcal{P}_{\mu}\left(\sigma^{\prime}\right) X^{\prime \mu}\left(\sigma^{\prime}\right) \partial_{\sigma} \delta\left(\sigma-\sigma^{\prime}\right) \text {. }
\end{aligned}
$$

Logo,

$$
\left\{\varphi_{1}(\sigma), \varphi_{1}\left(\sigma^{\prime}\right)\right\}=\left[\varphi_{1}(\sigma)+\varphi_{1}\left(\sigma^{\prime}\right)\right] \partial_{z} \delta\left(\sigma-\sigma^{\prime}\right)
$$

Agora vamos considerar 0 parênteses de Poisson entre $\varphi_{1}$ e $\varphi_{2}$ :

$$
\begin{aligned}
& \left\{\rho_{1}(\sigma), \varphi_{2}\left(\sigma^{\prime}\right)\right\}=\left\{\mathcal{P}_{\mu}(\sigma), \bar{G}^{\alpha^{\prime} \beta^{t}}\left(\sigma^{\prime}\right)\right\} X^{\alpha^{\mu}} \mathcal{P}_{\mathbf{a}^{\prime}}\left(\sigma^{\prime}\right) \mathcal{P}_{\beta^{\prime}}\left(\sigma^{\prime}\right) \\
& +P(\sigma)\left\{X^{*}(\sigma), P_{\alpha^{\prime}} \mathcal{P}_{g^{\prime}}(\sigma)\right\} \bar{G}^{\alpha^{\prime} \beta^{\prime}}\left(\sigma^{\prime}\right) \\
& +\left\{P_{\mu}(\sigma), X^{\prime \alpha} X^{\prime \beta} G_{\alpha \beta}\left(\sigma^{\prime}\right)\right\} X^{\prime \mu}(\sigma)
\end{aligned}
$$

Vamos reescrever cada termo de (C.40): 


$$
\begin{aligned}
& \left\{P_{\mu}(\sigma), G^{\alpha^{\prime} \beta^{\prime}}\left(\sigma^{\prime}\right)\right\}=-\partial_{\mu}^{\left(\sigma^{\prime}\right)} \bar{G}^{\alpha^{\prime} \beta^{\prime}}\left(\sigma^{\prime}\right) \delta\left(\sigma-\sigma^{\prime}\right)
\end{aligned}
$$

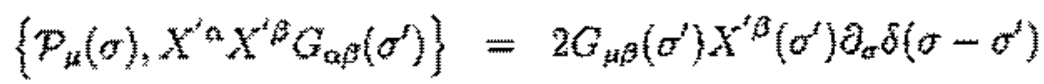

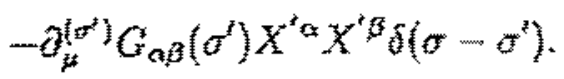

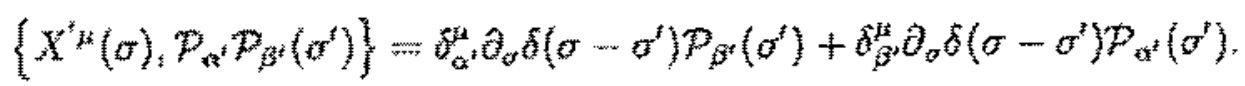

Consideremos as seguintes contribuiçöes :

$$
\begin{array}{r}
-\partial_{\beta}^{\left(\sigma^{\prime}\right)} G_{\alpha \beta}\left(\sigma^{\prime}\right) X^{\prime \alpha}\left(\sigma^{\prime}\right) X^{z \beta}\left(\sigma^{\prime}\right) X^{\prime \mu}\left(\sigma^{\prime}\right) \delta\left(\sigma-\sigma^{\prime}\right)= \\
-\partial_{\sigma^{\prime}} G_{\alpha \beta}\left(\sigma^{\prime}\right) X^{\prime \alpha}\left(\sigma^{\prime}\right) X^{\prime \beta}\left(\sigma^{\prime}\right) \delta\left(\sigma-\sigma^{\prime}\right)
\end{array}
$$

Porém, para uma funçäo $f(\sigma)$ :

$$
\begin{aligned}
f\left(\sigma^{\prime}\right) \partial_{\sigma} \delta\left(\sigma-\sigma^{\prime}\right) & =\partial_{\sigma} f(\sigma) \delta\left(\sigma-\sigma^{\prime}\right)+f(\sigma) \partial_{\sigma} \delta\left(\sigma-\sigma^{\prime}\right) \\
-\partial_{\sigma} f(\sigma) \delta\left(\sigma-\sigma^{\prime}\right) & =\left[f(\sigma)-f\left(\sigma^{\prime}\right)\right] \partial_{\sigma} \delta\left(\sigma-\sigma^{\prime}\right)
\end{aligned}
$$

Constderando $f(\sigma)=\sigma_{n \beta}(\sigma) X^{*} \alpha(\sigma) X^{*} \beta(\sigma)$ temos:

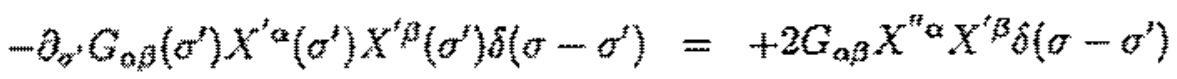

$$
\begin{aligned}
& +\left[f(\sigma)-f\left(\sigma^{t}\right)\right] \partial_{\sigma} \delta\left(\sigma-\sigma^{t}\right)
\end{aligned}
$$

Entäo, com $(C .44)$ e (C.46) temos: 


$$
\begin{array}{r}
\left.-\partial_{\beta}^{f} \alpha^{\prime}\right) G_{\alpha \beta}\left(\sigma^{\prime}\right) X^{\prime \alpha}\left(\sigma^{\prime}\right) X^{\prime} \beta\left(\sigma^{\prime}\right) X^{\prime} \alpha\left(\sigma^{\prime}\right) \delta\left(\sigma-\sigma^{\prime}\right)= \\
2 G_{\alpha \beta}\left(\sigma^{\prime}\right) X^{* \prime \alpha} X^{\prime \beta}\left(\sigma^{\prime}\right) \delta\left(\sigma-\sigma^{\prime}\right)+\left[f(\sigma)-f\left(\sigma^{\prime}\right)\right] \partial_{\sigma} \delta\left(\sigma-\sigma^{r}\right)
\end{array}
$$

Para analisarmos $2 G_{u g}\left(\sigma^{\prime}\right) X^{\prime} \beta\left(\sigma^{\prime}\right) X^{\prime \mu}(\sigma) \partial_{\sigma} \delta\left(\sigma-\sigma^{\prime}\right)$, devemos antes notar que:

$$
X^{z} \beta(\sigma) \partial_{\sigma} \delta\left(\sigma-\sigma^{\prime}\right)=-X^{n} \mu\left(\sigma^{\prime}\right) \delta\left(\sigma-\sigma^{\prime}\right)+X^{t} t\left(\sigma^{\prime}\right) \partial_{\sigma} \delta\left(\sigma-\sigma^{\prime}\right)
$$

onde usarnos $(C .45)$.

Então:

$$
\begin{aligned}
& 2 G_{i t}\left(\sigma^{\prime}\right) X^{*}\left(\sigma^{\prime}\right) X^{*} \mu(\sigma) \partial_{\sigma} \delta\left(\sigma-\sigma^{\prime}\right)= \\
& 2 \sigma_{\mu \beta}\left(\sigma^{\prime}\right) X^{\prime \beta}\left(\sigma^{\prime}\right) X^{\prime \mu}\left(\sigma^{\prime}\right) \partial_{\sigma} \delta\left(\sigma-\sigma^{\prime}\right)-2 G_{\alpha \beta}\left(\sigma^{3}\right) X^{t \beta}\left(\sigma^{\prime}\right) X^{\mu} \mu\left(\sigma^{\prime}\right) \delta\left(\sigma-\sigma^{\prime}\right)
\end{aligned}
$$

Somando (C.49) e (C.47) obtemos:

$$
\left.\left[G_{k \beta \beta}\left(\sigma^{\prime}\right) X^{\prime \beta}\left(\sigma^{\prime}\right) X^{t}+\sigma^{\prime}\right)+G_{\mu \beta}(\sigma) X^{*} \beta(\sigma) X^{\prime \mu}(\sigma)\right] \partial_{s} \delta\left(\sigma-\sigma^{\prime}\right)
$$

Una outra contribuiçăo em (C.40) ê dada por:

$$
\begin{aligned}
& -\partial_{j k}^{\left(\alpha^{\prime}\right)} \sigma^{\alpha} \alpha^{t} \delta^{\prime} X^{\prime}\left(\sigma^{\prime}\right) p_{\alpha^{\prime}}\left(\sigma^{\prime}\right) p_{\beta^{\prime}}\left(\sigma^{\prime}\right) \delta\left(\sigma \cdots \sigma^{\prime}\right)= \\
& -\partial_{z^{\prime}} \tilde{G}^{\alpha^{\prime} \beta^{i}}\left(\sigma^{\prime}\right) \mathcal{P}_{\alpha^{\prime}}\left(\sigma^{\prime}\right) \mathcal{P}_{\beta^{\prime}}\left(\sigma^{\prime}\right) \delta\left(\sigma-\sigma^{\prime}\right)
\end{aligned}
$$

Considerando que $g(\sigma)=C^{3} a^{\prime} \beta^{\prime}(\sigma) P_{\alpha^{\prime}}(\sigma) P_{\beta^{\prime}}(\sigma)$ temos a partir de $(C .45)$ :

$$
\begin{aligned}
-\partial_{\sigma} \tilde{G}^{\alpha^{\prime} \beta^{\prime}}(\sigma) P_{\alpha^{+}} \mathcal{P}_{\beta^{\prime}}(\sigma) \delta\left(\sigma-\sigma^{\prime}\right) & =2 \tilde{G}^{\alpha^{\prime} \beta^{\prime}}(\sigma) \partial_{\sigma} p_{\alpha^{t}}(\sigma) \mathcal{P}_{\beta^{\prime}}(\sigma) \delta\left(\sigma-\sigma^{\prime}\right) \\
& +\left[\sigma(\sigma)-g\left(\sigma^{\prime}\right)\right] \partial_{\sigma} \delta\left(\sigma-\sigma^{\prime}\right)
\end{aligned}
$$


A partir de $(C .52)$ e $(C, 51)$ obtemos

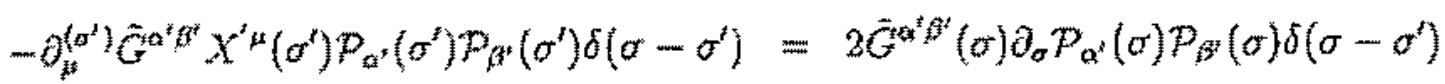

$$
\begin{aligned}
& +\left[g(\sigma)-g\left(\sigma^{\prime}\right)\right] \partial_{\sigma} \delta\left(\sigma-\sigma^{3}\right)
\end{aligned}
$$

Analogamente à $(\mathrm{C} .48)$, temos

$$
P_{\alpha^{1}}(\sigma) \partial_{\sigma} \delta\left(\sigma-\sigma^{\prime}\right)=-p_{\alpha^{\prime}}^{\prime} \delta\left(\sigma-\sigma^{\prime}\right)+P_{\alpha^{\prime}}\left(\sigma^{\prime}\right) \partial_{\sigma} \delta\left(\sigma-\sigma^{\prime}\right)
$$

Somando $(\mathrm{C} .54)$ e $(\mathrm{C} .52)$ obtemos

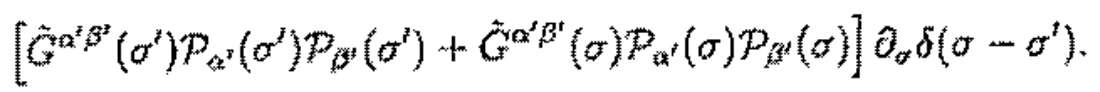

Entäo, considerando todas as contribuigots feamos com

$$
\left\{\varphi_{1}(\sigma), \sigma_{2}\left(\sigma^{\prime}\right)\right]=\left[\varphi_{2}(\sigma)+\varphi_{2}\left(\sigma^{\prime}\right)\right] \partial_{z} \delta\left(\sigma-\sigma^{5}\right)
$$




\section{Bibliografia}

[1] S. W. Hawking e R. Penrose, Proc. Roy. Soc. Lond. A314, 529 (1970); S. W. Hawking and G.F. R. Ellis, The Large Scale Structure of Space-time (Cambridge, 1973); G.T. Horowitz and R. Myers, Gen. Rel. Grav, 27 (1095) 915; R. H. Brandenberger, grqc/9503001 (näo publicado).

[2] A. Strominger e E. Martinec, Phys. Lett, B379 (1906) 99; C.G. Callan, Jx. e J. M. Mallacena, Nuel. Phys. B472 (1096) 591; G. T. Horowitz e A. Strominger, Phys. Rev. Lett. $77(1996) 2368$.

[3] G. T. Horowitz e S. F. Ross, "Possible Resolution of Black Holes Singularities from Large N Gauge Theory", hep-th/9803085 e JHEP 0804 (1998) 015; A. Lawrence e E. Martinec, Class Quant. Grav, 13 (1996) 63.

[4] G. T. Horowitz and D. Marolt, Phys. Rey, D52 (1995) 5670

[5] G. 'T. Horowitz, Class. Quant. Grav. 8, 587 (1991) ; J. Louko e R. D, Sorkin, Class, Quant. Grav. 14 (1997) 179.

[6] A. Chamblin, Topology and Causal Structure gr-qc/9509046 (não publicado).

[7] J. Smallwood, J. Math. Phys. 20 (1979) 459.

[8] L. M. Sokolowski, Acta Phys. Pol. B6 (1975) 520, ihid. 657 
[9] 1. Bengtsson, Class. Quant. Grav. 8, 1847 (1991); T. Jacobson e J. D. Romano, Class. Quant. Grav, 9, L 119 (1992).

[10] Y. Ma, C. Liang e Z. Kuang, Class. Quant. Grav, 16(1999) 605; ibid., Phys. Rev. D59 (1990) 044008 .

[11] A. Achúcarro e P. Townsend, Phys. Lett B 180, 89 (1986); E. Witten, Nucl. Phys. B 311, 46 (1988); K. Ister e C. Trungenberger, Phys. Rev. Lett. 63, 834 (1989); A. Chamseddine a D. Wyler, Phys. Lett. B228, 75 (1989); D. Cangemi e R. Jackiw, Phys. Rev. Lett: 69, 233 (1992).

[12] M. Blau e G. Thompson, Ann. Phys. 205 (1901) 130.

[13] E. Witten, Comm. Math. Phys. 121 (1089) 351.

[14] E. Witten, Comm. Math. Phys. 117, 353 (1988).

[15] J.C. Baez, Commun. Math. Phys. 193 (1998) 219.

[16] O. A. Mattos e V. O. Rivelles, Phys, Rev. Lett. 70, 1583 (1993).

[17] R. P. Zaikov, Phys. Lett. B263 (1991) 206.

[18] A. Karlhede e U. Lindstrom, Class. Quant. Grav. 3 (1986) L73.

[19] U. Lindstrom e R. von Unge, Phys. Lett. B403 (1997) 233; H. Gustafsson e U. Lindstrom, Nucl. Phys. B540 (1999) 520 .

[20] D. M. Marolf, Class. Quant. Grav. 11 (1994) 239.

[21] L.A. Cabral e V.O. Rivelles, Particles and Strings in Degenerate Metric Spaces, preprint 1999. 
[22] H. Kleinert, Path Integrals in Quantum Mechanics, Statisties and Polymer Plysics (World Scientific, Singapore, Second Edition, 1995).

[23] P.A.M. Dirac, Lectures on Quanttum Mechanics (Yeshiva University Press, NY, 1964).

[24] D. Lovelock e H. Rund, Tensors, Differential Forms and Variational Principles (JOHN WILEY \& SONS, New York, 1975).

[25] C. W. Misner, K.S. Thorne J.A. Wheeler, Gravitation (W.H.FREEMAN and Company, San Francisco, 1973).

[26] K. Sundermeyer, Coustrained Dynamics (Springer-Verlag, 1982).

[27] M. Green, 3. Schwartz e E. Witten, Supetstring Theory (Cambridge U. Press, NY, 1987).

[28] A. Giveon e M. Rocek, Introduction to Duality, hep-th/9406178.

[29] A. Giveon e M. Rocek, Nucl. Fhys. B421 (1994) 173.

[30] T. Buscher, Phys. Lett. B194 (1987) 59.

[31] M.Diakonou, K.Farakos, G.Koutsoumbas e E.Papantonopoulos, Phys. Lett. B240 (1990) 351 ; ibid. B247 (1900) 273.

[32] 1.Wess, Nucl. Phys. B15(Proc. Suppl.) (1090) 35.

[33] C.G.Callan, D. Friedan, E.J. Martinec e M.J. Perry, Nucl. Phys. B262 (1985) 503. 